\title{
The Leadership Processes of Pacific Public Servants in Aotearoa, New Zealand
}

\author{
By \\ Mele Katea Paea \\ A thesis \\ submitted to the Victoria University of Wellington \\ in partial fulfilment of the requirements for the degree of \\ Master of Management Studies
}

Victoria University of Wellington

2009 


\begin{abstract}
This dissertation presents research focused on leadership processes among Pacific public servants at multiple levels in the New Zealand Public Service. The current study was guided by this research question: What are the leadership processes currently employed by Pacific public servants in the New Zealand Public Service? This study also explored participants' views on the effect of Pacific cultural backgrounds and organisational contexts on their current experience of leadership processes.
\end{abstract}

The exploration of the topic was developed within a post-positivist research paradigm, using phenomenological methodology to examine the leadership processes of Pacific public servants. It employs qualitative case studies of two New Zealand Public Service organisations in the Wellington region. I employed two data collection tools in these case studies. The first was the use of in-depth interviews, and the second was an analysis of relevant organisational documents. A total of sixteen Pacific public servants participated in my study, eight from each case organisation.

The findings indicated that the Pacific participants understood leadership as a social process of collective influence within a context. Participants perceived participating, networking and relationship building, learning about leadership from cultural contexts, and practising the Pacific value of $v a$ as important leadership processes for their performance in the organisations in which they were working. This study also found that the organisations' key roles and leadership values, which are embedded in Pacific cultures, shaped participants' experiences of the leadership processes. The findings also highlight some factors that contribute to and constrain the Pacific public servants' leadership processes. This emphasises the need for diverse policies to encompass leadership development. 
This study also highlights the need for leadership support for Pacific public servants at all levels in their New Zealand organisations. Practical and future research recommendations gained from the findings are discussed. The study contributes to the field of leadership research on Pacific public servants in New Zealand, and provides a different perspective on leadership processes in general leadership theory. 


\section{ACKNOWLEDGEMENTS}

My sincere thanks to Dr. Deborah Jones and Sarah Proctor-Thomson for their supervision of this study, particularly for their valuable feedback on the theoretical and practical aspects of the research. I also thank the NZAID - New Zealand's International Aid and Development Agency for full financial support of my study. Special thanks must go to the sixteen Pacific public servants who gave freely of their time to partake in my study and allowed me to share their leadership experience in the Public Service. I would also like to thank my Pacific Learning Adviser, Ruth Davidson-Toumu'a, for her encouragement and additional learning advice; and to all my colleagues who shared with me their experience. Finally, to my family for making everything possible to complete this study. Words cannot express enough gratitude for the love you have contributed throughout the study. Thank you to my parents and siblings who have always been there for me. I am also thankful especially to my husband and our children for their understanding and wholehearted support. Without your love, I would have had no time to fulfil this dream. 


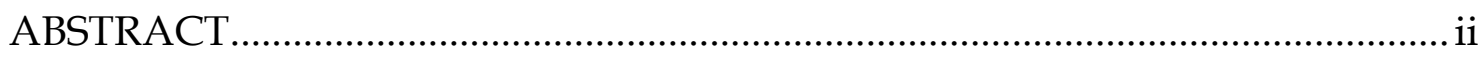

ACKNOWLEDGEMENTS .............................................................................. iv

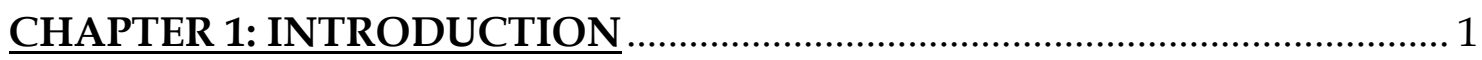

The research question and objectives ................................................................. 1

The rationale for my interest in the topic ......................................................... 2

The Pacific people in New Zealand and the public service ................................ 4

The research design ........................................................................................ 7

The significance of my study ………………………….................................... 9

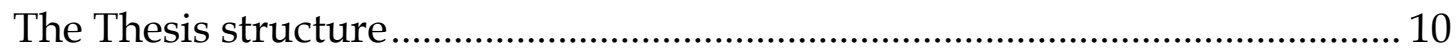

CHAPTER 2: REVIEW OF LITERATURE RELATED TO THE STUDY ......... 11

Definition of leadership...................................................................................... 11

Leadership process and theories......................................................................... 13

Minority ethnicity and leadership .................................................................... 21

Leadership in the Pacific nations ........................................................................ 23

Leadership process in the public service ............................................................. 26

Studies of Pacific public servants in the New Zealand Public Service.............. 32

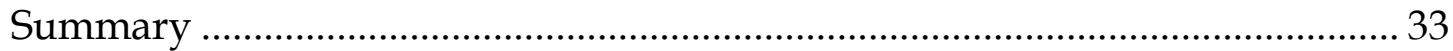

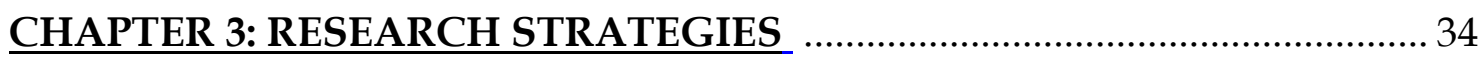

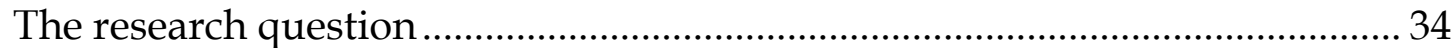

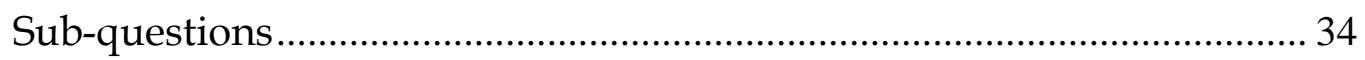

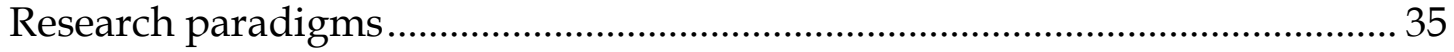

Research methodology ……………………………................................... 37

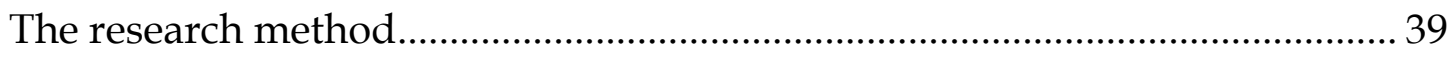

Case study research .................................................................................... 39

Participant population, setting, and participant sample........................... 41

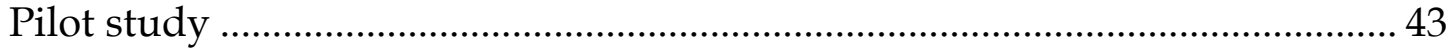




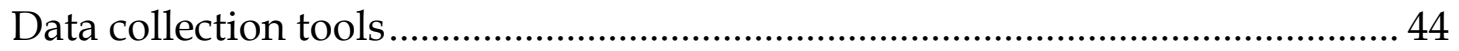

In-depth interview procedures ................................................................. 45

Document analysis procedure...................................................................... 47

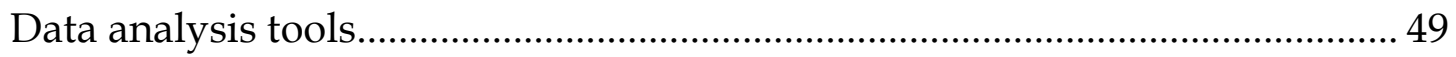

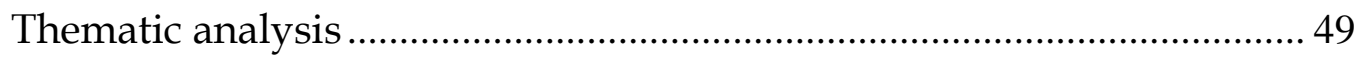

The process of final categorisation …………………….............................. 50

Research credibility ................................................................................... 54

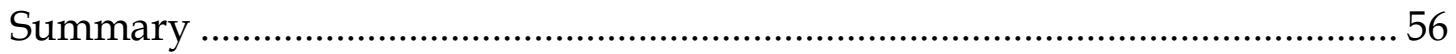

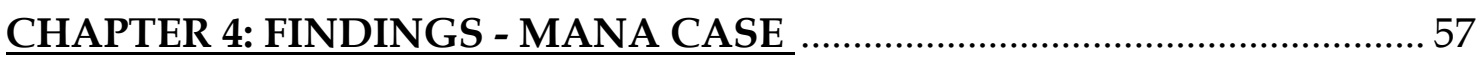

The organisational leadership processes ………………………………………... 57

The context of the Mana organisation............................................................ 57

The participating processes ......................................................................... 59

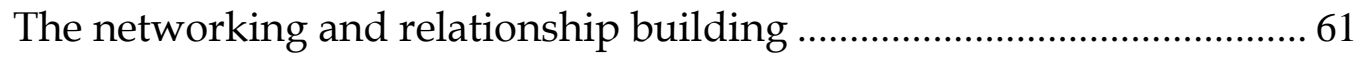

The possible constraints on organisational leadership processes............ 65

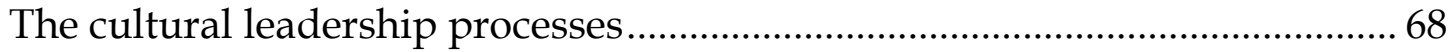

Learning about leadership from cultural contexts ........................................ 68

Practising the Pacific value of respect ......................................................... 73

Participants' perceptions of leadership in general ...................................... 74

Working in a non-Pacific context............................................................ 77

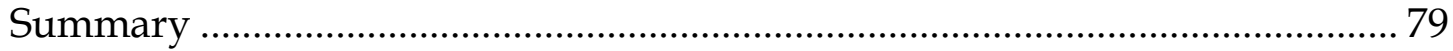

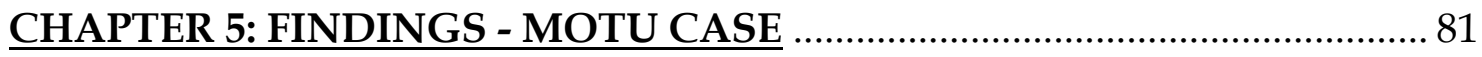

The organisational leadership processes ……………......................................... 81

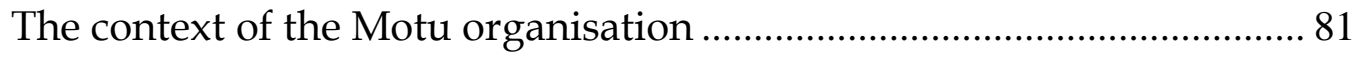

The participating processes ...................................................................... 82

The networking and relationship building ……………………………..... 85

The possible constraints on organisational leadership processes ............ 89

The cultural leadership processes........................................................................ 90

Learning about leadership from cultural contexts .................................... 90

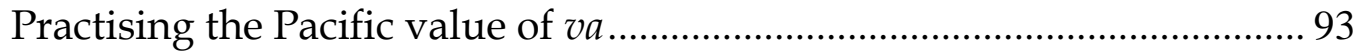


Participants' perceptions of leadership in general ................................... 97

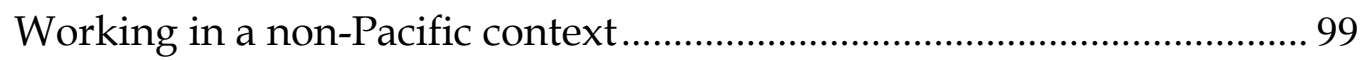

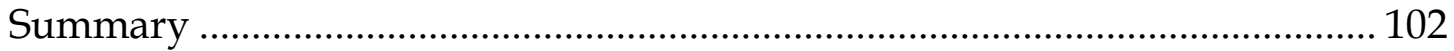

\section{CHAPTER 6: COMPARATIVE ANALYSIS AND THEORETICAL}

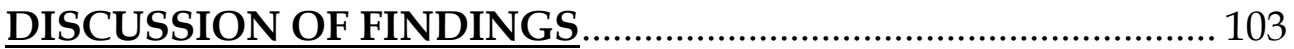

The comparative analysis and theoretical discussions .................................. 103

The participating processes ….................................................................. 103

The networking and relationship building .......................................... 106

The possible constraints on organisational leadership processes ......... 109

Learning about leadership from cultural contexts ................................ 112

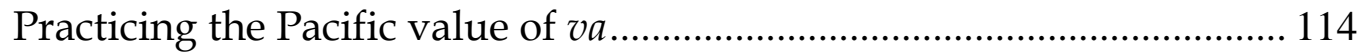

Participants' perceptions of leadership in general ................................. 115

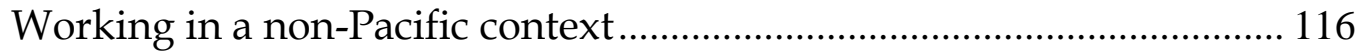

The conceptual framework of participants' leadership processes .................. 118

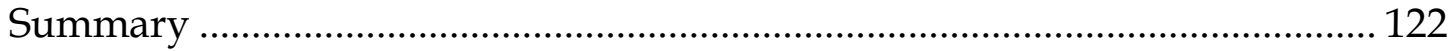

CHAPTER 7: CONCLUSION AND RECOMMENDATIONS …................... 124

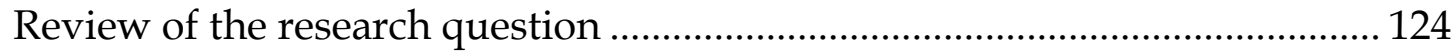

My reflections on the current study …..................................................... 127

Recommendations of support for Pacific public servants............................. 128

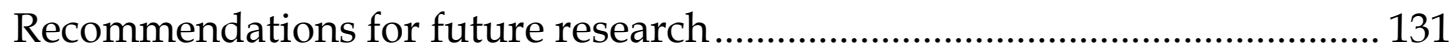

The contribution of my research to the leadership processes generally ........ 133

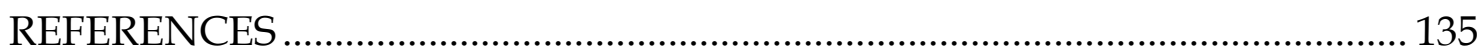

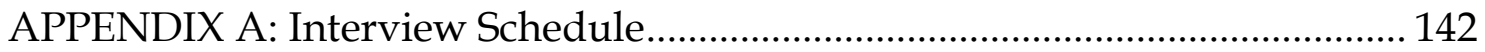

APPENDIX B: Information Sheet for Participants ........................................... 144

APPENDIX C: Participation Consent Form …................................................... 146

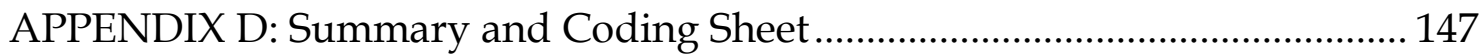




\section{LIST OF TABLES}

Table 1: Representation of Pacific people in the public service compared to the employed labour force in New Zealand 5

Table 2: The background information of Pacific participants by organisation.

Table 3: Category development of Pacific participants' leadership processes 52

Table 4: The organisational and cultural contributing and constraining factors of Pacific participants' leadership processes. 121

\section{LIST OF FIGURES}

Figure 1: The conceputal framework of Pacific participants' leadership processes in the New Zealand Public Service organisations 119 
Chapter 1

\section{INTRODUCTION}

This thesis aims to seek a better understanding and to raise awareness about the leadership processes currently employed by Pacific public servants in New Zealand, a non-Pacific context. It does so by examining the leadership process experiences of sixteen participants of Pacific Island descent in two public sector organisations in the Wellington region. The exploration also included participants' perceptions of the effect of their cultural background and the organisational contexts on their current leadership experiences being investigated. The broad focus of the current study in the initial stage was to simply study leadership processes in the public service. However, in the course of the study, the findings revealed the significant contribution of Pacific participants' leadership processes to the public service outcomes. This led to a greater focus on the leadership processes of Pacific public servants, as one group of minority public professionals in New Zealand.

The purpose of this chapter is to provide an overview of the current study. It begins by considering the core research question and objectives. It then describes the rationale for my personal interest in the topic, provides information about the Pacific people in New Zealand and the public service, and explains the research design for this study. The contribution of the study is discussed, and the thesis structure is outlined.

\section{The research question and objectives}

The question that guided the present research was: What are the leadership processes currently employed by Pacific public servants in the New Zealand Public Service? This question was developed to collect in-depth information for a better understanding of the participants' leadership processes in the two organisations in which they are operating. To answer the research question, three research objectives were established: 
1) To understand the leadership processes of Pacific participants which they perceived as important to their work and the organisational outcome;

2) To find out whether the participants' cultural background had any effect on their experience of leadership processes in the New Zealand context, and

3) To describe how the organisational context affects the Pacific public servants' leadership processes in New Zealand, as perceived by participants.

\section{The rationale for my interest in the topic}

My interest in this topic is driven by my professional experience as a public servant in a Pacific nation and a personal belief that the extent of the leadership process, as experienced by Pacific public servants in New Zealand, is underresearched and should be explored due to increasing number of Pacific workers entering the public service. My interest was also complemented by my postgraduate academic development and the fact that studying this useful topic is directly relevant to the career I hope to pursue. Deciding to research this topic has reinforced my personal interest and commitment to public servants who have useful and valuable leadership experiences in New Zealand organisations, but whose experiences and voices are seldom heard.

From my working experience in a Pacific Public Service organisation, leadership is based on formal organisational positions. The hierarchical style of leadership such as using positional power for controlling staff members to take action is practised. In six years of work experience, I witnessed many public servants who suffered from problems that plague the public service in general. These include staff dissatisfaction about how decisions are made in relation to the reshuffling of positions and benefits, high staff turnover, and to a large extent, low staff morale. These problems have led to an inefficient and ineffective organisation. It was a concern to me as a public servant because the hierarchical leadership appeared to lack emphasis on inspiring staff members to strive willingly to achieve organisational goals, and demonstrated little consideration of how employees feel and respond to such a leadership style. From a critical perspective, Knights and Willmott (2007) argue that occupying 
leadership in a hierarchical structure forces people to suppress potentially conflicting ideas or viewpoints. In other words, Knights and Willmott say that leadership in formal positions suppresses the leadership potential embedded in staff members.

When I started my postgraduate studies in New Zealand in 2005, I continued to reflect on the above problems. In the first component of my Masters programme in 2006, my interest in leadership was not manifested at the time; this may have been due to lack of leadership emphasis in the six management courses I took in 2006. In November 2006, I was one of thirty scholars who participated in the NZAID's ${ }^{1}$ Pacific Scholar Leadership Programme in Wellington, New Zealand. This programme is one of the NZAID's priorities to support leadership as a basis for providing vision and direction to strengthening governance in the Pacific Regions (NZAID, 2008). This is where my leadership journey began and the workshop had a stronger influence on my world view. The programme convinced me that leadership is for everyone, regardless of what position they are holding. It also promoted the importance of having courage and determination to get along with people of any institution. This idea of leadership links well with leadership as a process.

Bratton, Grint and Nelson (2005) argue that leadership is not a position but it is a process based on interaction between leaders and followers in the context in which they are operating. In my view, the word process implies that leadership is an action that is participated in by all members in the organisation. Evidence suggests that leadership is a process because it is about doing (Parry, 2002), relating (Rost, 1993), transforming (Burns, 1978), inverse leading (Grint, 2005), and influencing (Elkin, Jackson, \& Inkson, 2004). I describe these lived experiences as the people's world of -ing such as doing, interacting, acting, and relating. This evidence emphasised the practical element of leadership as a verb or an action rather than as a noun or a person.

\footnotetext{
${ }^{1}$ New Zealand's International Aid and Development Agency
} 
Since joining the NZAID leadership workshop, my leadership interest started preparing me for the big picture of the present study. When I started the groundwork for this study in late 2007, I wanted to research something useful and relevant to my intended career. In fact, I began reflecting on the experience I had in the public service and how I could contribute to improving the situation. Initially, I wished to conduct this study with the public service in my home country, but this was not possible due to communication problems and time restraints. These problems, however, did not inhibit my interest in conducting the research in a public service context.

After several discussions with academic staff about my research interests, I decided to conduct my study with Pacific public servants in New Zealand. Learning from the State Services Commission's (2004) report that Pacific people play an important role in public service outcomes but desire improvement in their leadership capabilities, this study certainly provides insight about possible leadership supports appropriate for them in New Zealand. Moreover, my experience and the findings from this research should assist me in thinking about the appropriate leadership development that may be useful for public servants in my home country. As the New Zealand and Australian governments place high priority on good governance in many Pacific nations through their development programmes in the Pacific region, this study contributes to achieving this goal with the understanding that Pacific leadership is central to those strategic visions.

The Pacific people in New Zealand and the public service

In New Zealand's 2006 Census, Pacific people living in New Zealand were estimated to number 266,000, out of a total New Zealand population of 4.1 million (Statistics, 2008a, 2008b). According to the Statistics (2008b) report, the Pacific population in 2006 had increased by 15 percent since the 2001 Census and 59 percent since the 1991 Census. This demonstrates that the Pacific people's ethnic group in New Zealand is a growing one. The largest ethnic group among the Pacific people of New Zealand identified in the 2006 Census 
was Samoans. The next largest groups were the Cook Islanders, Tongans, Niueans, Fijians, Tokelauans, and Tuvaluans.

In 2005, the report released by the Ministry of Pacific Island Affairs (MPIA, 2005) on Pacific leadership in the New Zealand Public Service illustrates that the Pacific public servants contribute to about 7.1 percent of the public service in 2004, equivalent to 2,373 public servants. The report also indicates that the representation of Pacific workers in the public sector increased from 6.3 percent in 2000 to 7.1 in 2004. It is projected that by 2010, Pacific public servants will increase to 13 percent. With the exception of the Tuvaluans, Pacific staff in the public service are represented by six Pacific ethnic groups; Samoans, Cook Islanders, Tongans, Niues, Fijians, and Tokelauans (SSC, 2004). For the purposes of my study, those who self-identify as belonging to one or more of the six major Pacific ethnic groups are defined as part of this 'Pacific' group of peoples.

Pacific people in New Zealand are over-represented in the public service compared with other industries (SSC, 2004). Table 1 shows the representation of Pacific people in the New Zealand Public Service and in the wider employed labour force between 1998 and 2003.

\begin{tabular}{|l|c|c|c|c|c|c|}
\hline Pacific people & $\mathbf{1 9 9 8}$ & $\mathbf{1 9 9 9}$ & $\mathbf{2 0 0 0}$ & $\mathbf{2 0 0 1}$ & $\mathbf{2 0 0 2}$ & $\mathbf{2 0 0 3}$ \\
\cline { 2 - 7 } & $\mathbf{9}$ & $\mathbf{0}$ & $\mathbf{0}$ & $\mathbf{0}$ & $\mathbf{\%}$ & $\mathbf{\%}$ \\
\hline Public Service & 5.9 & 6.2 & 6.6 & 6.6 & 6.8 & 7.1 \\
\hline Employed Labour Force & 3.6 & 4.0 & 4.0 & 4.0 & 4.5 & 4.5 \\
\hline
\end{tabular}

Table 1: Representation of Pacific people in the public service compared to the employed labour force (MPIA, 2005).

As the numbers of Pacific people in the New Zealand Public Service grows, so too does their concern for recognising their cultural values (MPIA, 2005; SSC, 2004). The State Services Commission's (2004) report on Pacific people as part of its role to promote 'Equal Employment Opportunity' for all public servants, 
highlighted barriers that Pacific public servants faced while working in New Zealand. Among others, these problems included difficulties in recognition of their cultural values as important skills that contribute to the workplace, having fewer opportunities for their direct input into decision-making, and a lack of development opportunities for them in the public service. These problems also show the importance of their cultural values to their performance in non-Pacific organisations.

The State Services Commission (2004) report also provided strategies suggested by Pacific public servants to address the issues. These strategies included: acknowledging cultural skills in the organisational strategies and recognising them for performance appraisal, setting up formal mentoring schemes, providing training for non-Pacific staff on cultural issues, monitoring the organisation's cultural environment, and developing a public service job experience scheme (SSC, 2004, p. 5). These proposals are not new to the development of Pacific public servants because they have existed for 15 years in a handbook by the State Services Commission in 1993. They were developed following a Cabinet decision in 1991 for Public Service Chief Executives to develop strategies for improving Pacific people's participation at all levels of the public service (SSC, 1993). Thus, problem about the Pacific people in the New Zealand Public Service was recognised but still exists.

The Ministry of Pacific Island Affairs' (2005) report on the Pathway Leadership Conference held in 2004 to promote Pacific public servants' leadership opportunities and effective participation in higher-level decision making, indicated similar barriers and practical implications to those mentioned above. This conference stressed that Government agencies needed to implement internal action plans that the Ministry of Pacific Island Affairs had developed for Pacific peoples' leadership development. The Ministry of Pacific Island Affairs' (2005) report was highlighted the Pacific leadership qualities of respect, relationship, and collectiveness. However, this finding gives little information 
about the Pacific workers' actual leadership experiences at all levels of the public service, and what leadership processes they believe to be important to their specific organisation in New Zealand. Although the emphasis on cultural values of Pacific leadership was strong, little is known about how Pacific public servants learnt about leadership from cultural contexts and how they applied their cultural leadership experience in New Zealand organisational contexts.

\section{The research design}

The data for the current study was obtained from two organisational case studies with a total of sixteen Pacific public servants, eight from each case organisation. These participants all work permanently in these two organisations within the Wellington region. Due to lack of information available on the research topic, the case study method was chosen to collect indepth information and to uncover multiple realities for better understanding of the situation. The main tools that were used for data-gathering in the case studies were the in-depth interviewing of participants and analysis of relevant public documents available from both organisations. The interviews were conducted to understand the leadership process experiences of the Pacific participants, and the document analysis was used to support the interview data and to understand the formally stated leadership strategies of the two organisations being studied.

This study does not attempt to make any changes in the performance of these two public service organisations, but rather to understand and describe the leadership processes currently employed by this particular group of people in New Zealand. These organisations were chosen out of my personal interest in their involvement with the leadership development of Pacific public servants in New Zealand. 
The face-to-face interview method was considered useful in my study for obtaining information and opinion, and allowing the researcher to take control of the process and to provide freedom for the participants to express their views. It is also the preferred and suitable research technique for researchers involving Pacific participants, as the realities underpinning the Pacific worldviews can be more easily revealed through conversation rather than writing (Anae, Coxon, Mara, Wendt-Samu, \& Finau, 2001). While Anae and colleagues' report was developed to give a set of research guidelines for researchers on Pacific education issues, central to my study is their finding that the "Pacific Way is spoken rather than written" (Anae et al., 2001, p. 14).

Prior to the actual process of data gathering, a pilot study was undertaken with two Pacific public servants who had characteristics similar to my intended Pacific participants to test the consistency of the research strategies. My reflections on this pilot study improved the design of data collection tools to better address the research question and objectives. The qualitative data gathered from the participants' interview and organisational documents were analysed and compared using thematic analysis tools through exploration of repeated words, phrases, concepts, and the interconnections of themes. The process of the categorisation of themes was developed using Constas's Documentation Approach for category development (Constas, 1992). This approach was chosen because the explicit details provided by Constas on how and when categories should be developed were useful for me to explain how themes in my study were developed. The full description of research strategies is provided in Chapter Three of this thesis. 


\section{The significance of my study}

A study of the leadership process experienced by public servants in public service organisations is crucial for movement and change but there is a lack of information available on this topic. It is particularly obvious that there is almost a complete absence of thorough research into leadership process experiences of Pacific public servants in a non-Pacific work context. Taking into account this gap in the leadership literature, my study raises awareness of various parties who are concerned with the performance of Pacific workers in the New Zealand Public Service.

At the heart of my research is the understanding of leadership specifically as a social process and this makes the research of leadership in the current study different from that in the general academic research of leadership processes in the public service. Researchers of leadership processes in the public service contexts, such as Van Wart (2005), have conducted their studies from the understanding that leaders are at the forefront of effective leadership process. As Murrel (1997) suggests, leadership can be viewed as social action that involves everyone within an organisational context. Just as Murrel does not distinguish between leaders and followers, I also believe that it is not meaningful to differentiate the people involved in leadership into groups called 'leaders' or 'followers'. This is because everyone in the organisation moves together towards achieving the organisational roles.

From my view, studying the social processes of leadership in the public service is necessary for public servants to come to value diversity and learn from a range of leadership experiences, and to understand the complexities of the leadership experiences by different people within the organisation. Such research would also enable decision-makers within the organisation to think about how to address these complexities in a sensitive ways for the benefit of the collective. 
The current research can be described as a founding piece of academic research designed specifically for understanding the Pacific workers' leadership processes at all levels within non-Pacific Public Service contexts. I hope that once the leadership experiences of these Pacific participants are more fully understood, the recommendations in my study may provide a starting point for how best to support and develop them in New Zealand. My study also represents the voice of Pacific public servants for decision makers to consider and presents the view that Pacific people in the public service are valuable resources whose leadership capabilities are worth enhancing and developing.

\section{The Thesis structure}

This thesis comprises seven chapters, including this introductory chapter. Chapter Two presents a review of the literature including leadership theories and studies relevant to the current study. Chapter Three describes details of research strategies involved in the current study. It presents the core research question and sub-questions that were used to guide the entire process, discusses the research paradigm, research methodology, and the methods. The research methods provide an explanation of how participants were selected and the tools used for data gathering, as well as procedures for data analysis.

Chapters Four and Five present the findings from the two organisational cases. Chapter Four describes the results of findings that emerged from the Mana case, and Chapter Five presents the findings emerged from the Motu case. Chapter Six consists of the comparative analysis of themes that emerged from both cases and links the key findings to existing leadership theories and studies on the issues involved. Chapter Seven concludes this thesis with an overall review of the research question and objectives in response to the general findings, my reflections on the current study, and provides suggestions for future actions and research. 


\section{Chapter 2}

\section{REVIEW OF LITERATURE RELATED TO THE STUDY}

Not all public servants begin their professional careers in the public service with the understanding that the social processes they do experience in the organisational daily activities are leadership actions. The majority of public servants, on the other hand, still perceive leadership from its traditional understanding as a position, and the best managerial behaviour of a leader. In my view, this is because prior researchers focused primarily on the implicit mainstream understanding of leadership processes that view leaders as origins of successful outcomes.

To provide a grounded understanding of leadership process appropriate for the current study, this chapter aims to explore existing leadership theories and studies related to the understanding of leadership processes and issues regarding Pacific leadership. The review intends to gain understanding of the topic and forms the basis for my research. This chapter begins with discussing what the literature says about the definition of leadership in relation to leadership process. The next section reviews what theorists and researchers say about the theories of the leadership process. It then continues to describe the literature on minority ethnicity and leadership, leadership in the Pacific nations, the leadership processes in the public service, and studies of Pacific public servants in the New Zealand Public Service.

\section{Definition of leadership}

A comprehensive review of the leadership literature confirms that there are almost as many definitions of leadership as there are different researchers attempting to define the concept (Stogdill, 1974). This lack of clear definition increases the gap of understanding leadership, leading most researchers to define leadership from their own interests. Rost (1993) argues that failing to agree on a precise definition does not add value to people's understanding 
about the significant concept of leadership. It was evident in his evaluation of the leadership definitions from 1900 to 1989 that about 62 percent, equivalent to 366 out of 587, of published leadership works from books, chapters, and articles had no leadership definition. This concern over the definitions of leadership indicates that the researcher of any given study must provide a clear definition of leadership to ground readers in the significant concept of leadership they wish to explore.

There are four components of defining leadership that are emphasised in the current study. The first aspect relates to the notion of social process or what Parry (1998) describes as a process of action which depends on people and their interactions with each other. What is important from Parry's definition is that there is no distinguishing between leaders and followers' actions in the leadership process because leadership is everyone's action. This links with Murrel's (1997) view of leadership as a social action. Secondly, leadership involves influencing others, or what Sanga and Walker (2005) describe as the ability of a person to inspire someone's thinking and action. This is consistent with what Bratton and his colleagues (2005) describe as actions of persuading one another to use their leadership potential to do the work they might not otherwise do.

Thirdly, leadership connects with the notion of collective involvement. This means there are multiple people engaged in the process of leadership, not just leaders themselves, and the relationships among them should be strengthened. This is an emerging discourse emphasising a shift from the usual understanding of leadership as individual efforts to a new perspective of collectively based leadership. With this in mind, there is a need for leadership researchers to put special emphasis on studying leadership as the "processes of how people decide, act and present themselves to each other" (Murrel, 1997, p. 39). Murrel believes that leadership is a collective process which involves actions of multiple people at different levels. In other words, leadership is not a 
one-way influencing process but a collectively based influencing process. In my view, this concept indicates that leadership is the process of making sense about what people are doing together and how they are moving together towards achieving a desired outcome. I believe that this way will encourage people in the organisation to understand and commit to what they are doing.

The final component is that leadership occurs within a context. Bratton and colleagues (2005) suggest that leadership is a process which occurs within a context. In other words, leadership exists in any context, and different circumstances of different organisational contexts play important roles in shaping the leadership processes that are appropriately considered within a particular situation. In a public service organisation, for example, leadership processes can be shaped by both external and internal factors such as political power and the organisational structure.

Despite multiple ways that leadership is conceptualised, the above definitions are common to the notion that leadership is a process depending on people's interactions within a context. The following components can be identified as central to the phenomenon of leadership in my study: process, people, influence, and context. For the purpose of the present study, leadership is defined as a social process of collective influence within a context. The next section of this chapter reviews what theorists and researchers say about the theories of the leadership process.

\section{Leadership process and theories}

Leadership has been the topic of much research worldwide. However, the understanding of leadership as a social process of collective influence in the organisational setting is not fully developed. Due to this lack of understanding of the leadership process, researchers in the last decade such as Yukl (2006) and Parry (1998) urge future research for a theoretical approach to leadership process, and the efficient use of the leadership potential of people. 
The interest in studying leadership process as a means of promoting relationship between leaders and followers can be traced back to studies in the 1970s. This relationship was generalised as a dyadic process. The dyadic process focuses on interactions between two people, a leader and a follower. One of the well-known theories of dyadic process was conceptualised by Burns (1978) as a transforming process. The transformational process refers to the process where leaders inspire followers to do more than they are usually expected to do. What is important about Burns' transforming process is that it brings about change by getting people to work together with certain motives and values. The essence of transformational theory for understanding of the leadership process lies in the influential characteristics of the dyadic relationship to promote motive, trust, respect, and mutual obligations between the two parties (Bass \& Riggio, 2006).

Whilst the dyadic perspectives appear to limit the scope of leadership process to a single relationship between two people only, central to the present study is the follower's and leader's abilities to help and support each other to achieve the organisational goals. In relation to leadership process, the Transformational theory adds value to the sharing responsibilities between a leader and a follower. However, since the current study has conceptualised leadership as a process of collective influence, it is insufficient to say that only two people are involved in the leadership processes. As the dyadic process does not provide a clear description about the level of contribution from each side, which I assume to be that one is contributing more than the other, it is therefore necessary to describe the position of group processes in the leadership process.

In the late 1980s to early 1990s, there was a drift to the need to give more accountability for important activities of group leadership process. The group process refers to the interactions among group or team members who work together towards a shared outcome (Yukl, 2006). In essence, the group process is grounded on collective processes such as sharing ideas, skills, and 
responsibilities. In their studies of group members' abilities to interact collectively in the group activities, Podsakoff and his colleagues (1997) found a high level of group productivity when the level of shared commitments by members is high. In fact, the commitment of a group to share activities is central to the leadership process in acknowledging everyone's contribution to the group's success. Group processes contribute to the current study with the notion that leadership is a process of collective involvements.

The dyadic and group process approaches in leadership theory, both confirm leadership as a process of interactions among people through participating, building relationships, and sharing leadership skills. However, both approaches fail to consider the organisational context in which leadership is carried out by limiting the interaction to only two people or to members within a group. Hill (2004) argues that the major problem with the group process is that it does not apply an understanding of how an organisation behaves. As dyadic and group leadership processes both fail to consider that they exist within a larger social system, the organisational leadership processes should be emphasised.

From the late 1990s onward, interest on studying organisational leadership as a process emerged, taking into account the fact that staff members' interactions are occurred within an organisation. Organisational leadership researchers such as Bratton and colleagues (2005) propose that leadership is a process. This was illustrated in their tri-axis model showing that leadership process in the organisation depends on three critical factors: followers, leaders, and the context in which leaders and followers operate. In essence, it is a combination of peoples' actions at all levels in the organisation, and their leadership processes are employed for the purpose of influencing one another to move together towards achieving the organisational desired outcomes. What is important in this tri-axis model is that successful leadership processes have been redefined more broadly to move away from a single position role or person to a process that depends on equal power of organisation members. 
Although Bratton and his colleagues (2005) did not provide clear information about the types of appropriate leadership processes for an organisation, the follower component in their tri-axis model raised a point that everybody involved in the leadership process is important. Crucial to my study is the emphasis on followers' attributes, learning styles, and motivation that affect their interactions with leaders. This tri-axis model takes the position that followers are a critical component of the leadership process. Grint (2005) insists that followers make leaders, meaning that if followers are not involved in the leadership process, then there is no leadership. What is significant here is the extent to which leaders receive feedback from their followers.

While the context part of Bratton and his colleagues' (2005) tri-axis model is huge and may be a thesis on its own, important to my study is what these authors emphasised that leadership is shaped by internal and external factors of the organisational contexts. Bratton and colleagues described that the continuous changing of economic, political, and technological contexts also influence how leadership is undertaken in organisations; in terms of how the organisational strategies are formulated, how the organisation is structured, and how staff members are controlled as well as their adaptation to the changing environment. Yukl (2006) asserts that leadership processes in an organisational context are yet to be understood comprehensively. He adds:

More research is clearly needed on collective processes and contextual factors that determine leadership effectiveness in teams, organizations, and interorganizational joint ventures (Yukl, 2006, p. 451).

While surprisingly little work has been undertaken on leadership processes from the aspect of collective influence in the organisational context, significant developments have been made with leadership processes in organisations. An important contribution to the understanding of leadership processes in public service organisations may be linked with the Leadership Action Cycle theory developed by Van Wart (2005). In his attempts to provide a full coverage of the 
most important theoretical approaches to leadership in the public service, Van Wart emphasised three leadership processes which are appropriate for people behaviour in the organisation. The first is tasks-oriented, second is driven by the organisational processes, and the third is the people-oriented process.

Van Wart's (2005) tasks-oriented processes of leadership focus on promoting staff members participating in the organisational processes by employing delegation, sharing decisions, meetings, and consultations as instruments to get the views of the collective. This process of allowing people to participate in the organisational activities has been described as "optimizing" (Irurita, 1996, p. 129). Irurita's research on examining the social processes inherent in the nursing leadership situation found optimizing to be the key process for maximising the leadership capability of people. Irurita said:

Optimizing was the label given to the process of making the best of the situation, making the most effective or optimal use of all available and potential resources to compensate for the state of retardation and to move beyond mediocrity toward excellence (1996, p. 129).

It appears that optimizing is a social process of enhancing the effectiveness of collective involvement and making use of the qualities the organisation possesses from every member of the organisation. It is also worthwhile noting that Irurita did not suggest the optimization of the leadership qualities of only a few or some defined positions but "all available and potential resources" (Irurita, 1996, p. 129). The term "all" values the contribution and potential of every single member in the organisation. The types of human resources that Irurita stresses should be optimized included "knowledge, skills, attitudes/values, and beliefs ... self-esteem, energy, and confidence" (Irurita, 1996, p. 129). I also agree that allowing the participation of the collective to occur can motivate staff members to produce significant change in their performance or moving from an undesirable to a desirable situation. This is also known in the literature as participative leadership (Northouse, 2004). Northouse says that one of the key strengths of participative leadership 
approach is its practical aspect in emphasising the types of actions that are appropriate for motivating people to move as they go along to achieve the organisational goals.

Central to this study is the practical aspect of Van Wart's (2005) task-oriented processes to incorporate the collective views in the process of organisational decision-making. However, the influence process described in this approach is not from the collective works but from the leaders' efforts to practise these actions effectively with staff members. The task-oriented model does not show clear evidence about how the relationship among staff members is strengthened, and this may result from lack of empirical research to justify the validity of approaches similar to it such as participative and delegation leadership (Yukl, 2006).

Van Wart's (2005) second approach of leadership is the people-oriented processes. He emphasises here the soft competencies of the organisation and the contribution of motivating and developing staff to making a successful organisation, as he believes they are important for understanding ways of leading people. In my view, this approach shows the power of people in making organisations effective, which is concerned with the organisational processes that should be employed to develop staff capacities through respecting the inner qualities that drive their leadership capabilities.

Northouse (2004, p. 310) says that respecting peoples' ideas and considering them as human beings with "creative wants and desires" allow them to establish themselves in the organisation. MacMillan (2006) reflected that the major reason why his organisation performed successfully in 1951 to 1995 during his time as the Chief Executive Officer was that he had the power to build and sustain the social capital of the organisation. Although MacMillan claims that the soft concept of the organisation seems impossible to control, his 
ability to create opportunities for people to share ideas, providing long-term and continuous leadership support for his staff, motivated them to succeed.

Van Wart's (2005) people-oriented approach is comparable with the notion of aesthetic leadership (Hansen, Ropo, \& Sauer, 2007). Hansen and colleagues' aesthetic leadership is related to the process of respecting peoples' feelings and emotions as factual knowledge of expressing leadership. In my view, aesthetic leadership provides insight to look at the organisational leadership process beyond the actions that can be easily seen and to consider that feelings and emotions generate peoples' actions. Similar to Van Wart's people-oriented approach, aesthetic leadership emphasises the soft side of the organisation, which is the hidden qualities of leadership experience.

Van Wart's (2005) final approach is the organisational-oriented processes of leadership. This category describes various processes employed by the organisation to manage the organisational processes. From his list of the processes for organisational-oriented behaviours, the most significant process that is considerably important to my study is the leadership processes of "networking and partnering" (Van Wart, 2005, p. 250). This process is related to formal and informal means of developing contacts and building relationships among internal and external stakeholders. While informal networking and partnering processes were emphasised by Van Wart, most of those came from the organisation internal processes such as unscheduled meetings. However, little is known about the informal leadership processes of networking and partnering such as personal interactions and informal social networking by ethnic group. As Hosking (1988) said, social contacts and networking may be less active in the organisational context but may become more important when decision-makers use their social power to initiate such processes. 
The process of networking and partnering is compatible with sharing processes. Murrel's (1997) model of relational leadership conceptualised leadership as a collective process. He says:

Leadership is a social act, a construction of a "ship" as a collective vehicle to help take us as a group, organization or society desire to go ... Relational leadership puts the emphasis of study squarely on human processes of how people decide, act, and present themselves to each other. In this study, it is possible to see relationships other than those built from hierarchy and in those in which nurturing and supporting roles could be legitimized as means of influence (Murrel, 1997, pp. 3539).

From Murrel's relational perspective, leadership processes are founded on people interactions within a communal context, which form the basis of achieving the context's goals. Murrel looks at leadership differently, moving away from the leadership process of individuals. He emphasises three significant points central to the focus of my study. The first point is that leadership is an action, not a position. These actions or interactions among staff members are the key to making people move and produce significant change in their work. Secondly, leadership is a collective influence, not just a single person's job. In real meaning, leadership processes are actions built from the contribution of all members to achieve their desired outcome, not specifically from those who have power in their hierarchy positions. Finally, these social interactions may occur in any social context such as an organisation.

In general, while the above reviews of leadership theories build a picture of the various types of leadership processes appropriate for organisational contexts, there are limitations to the extent that they can be generalised. Researchers' approaches and the focus underlying their studies make a simple comparison impossible. What also emerges is that most research on leadership processes is based on person and outcome oriented approaches, meaning that they have been conducted from the perspective that a leader is the main source of effective leadership. 
Despite limitations on organisational leadership processes, I found that Van Wart's (2005) model generated ways of thinking about leadership processes, especially in a public service context. The review of this section found three leadership processes that are important in the organisational contexts: the taskoriented processes, the organisational processes, and the people-oriented processes. This list forms the basis of the investigation in my study which outlines the leadership processes taken up by Pacific public servants in the organisation contexts.

My study therefore incorporates an investigation of the leadership process for the purpose of understanding the Pacific public servants leadership processes crucial for their work in a non-Pacific context. In my study I have chosen to examine four key leadership processes commonly appeared in the above review, and are based on social processes of collective influence. They are the participating process, networking and relationship building, respecting of people, and learning about leadership. While these leadership processes, in general, were viewed from the organisation's broad behaviouristic way, my study also attempts to investigate the effects of ethnicity backgrounds on participants' leadership processes.

\section{Minority ethnicity and leadership}

The issue of cultural diversity and leadership has been addressed by many of the studies on international leadership research, largely on the responsibility of leaders in leading and managing diversity (Ballard \& Kleiner, 1988; Brain \& Lewis, 2004; Cox, 1991). However, research on the link between culture and leadership processes is yet to be studied comprehensively. In particular, effects of minority public servants' culture on their leadership behaviour in foreign public service organisations have not been investigated. With the rapid growth of globalisation and population, this concern has emerged as an area of research interest. Although it is not practical for all leaders to know the many cultures that exist in any one organisation, Ballard and Kleiner's (1988) understanding of managing issues regarding minority employees in America suggests that 
developing a universal cross-cultural programme of leadership is important to foster the leadership potential of all staff.

To fully utilise the diverse skills and values of workers, it is important to eliminate inhibiting factors that prevent the leadership potential of staff members (Cox, 1991). Using information on managing diversity available from the American organisations, Cox developed a multicultural organisation model which suggests ways to capitalise benefits and minimise costs of diversity experience by staff members of the organisation. These include i) focusing on skills rather than bias between culture identity and job status; ii) ensuring the contribution of minority-group inputs in the decision-making process and supporting the use of informal strategies such as mentoring and social networking to support minority; iii) providing equal distribution of human resources development; and iv) minimising personal conflicts by taking into account the feedback and views of diverse staff members (Cox, 1991, p. 41). It is obvious from Cox's study that in order to support minority members of any organisation, it is essential to consider the leadership skills they bring with them to a foreign organisational context, and to provide leadership support appropriate for them in that particular context.

Cox's (1991) arguments are similar to that put forth by Pfeifer (2006) regarding cultural diversity in New Zealand. Pfeifer suggests several factors that New Zealand managers must be taken into account to provide leadership that is appropriate to meet the needs of diverse cultures within New Zealand. These include leaders' ability to learn about other cultures, how to incorporate leadership experience from cultural contexts into the mainstream organisations, and full understanding of people within the organisation (Pfeifer, 2006, p. 38). Pfeifer's suggestions respond well to the issue of cultural diversity in Australia. Brain and Lewis's (2004) exploratory study of multicultural employees within an Australian government department found that the Australian supervisors lacked understanding of the leadership styles appropriate for a minority 
behaviour. Brain and Lewis's study also found that the minority group of nonAustralian employees experienced a high level of individualism and larger power distance cultures compared with the Australian employees.

It is apparent that the key leadership process appropriate for diversity is bringing people together by learning about the leadership values of different cultures, seeing the possibility for applying these values in formal organisations, and bearing in mind that leadership success is attributed to the collective rather than to the individual. In an article on Pacific public servants in New Zealand organisations, Ah Chong and Thomas (1997) offer some explanations on differences between the leadership behaviour of Pacific and Palangi ${ }^{2}$ people. They note that in New Zealand many Pacific workers are likely to prefer the leadership style of their Pacific leaders. As a result of their study, Ah Chong and Thomas found that followers' satisfaction depended on whether the leader was Pacific or Palangi. This difference between Pacific and Palangi style of leadership prompted to discuss examples of leadership in the Pacific nations that might help with understanding the link between ethnicity and leadership from a Pacific perspective. This is discussed next.

\section{Leadership in the Pacific nations}

While broad differences exist among the ways in which leadership is exercised in the Pacific nations (Churney, 1998), and regardless of the chieftainships and hierarchical nature of social organisations, a review of the literature available on leadership in the Pacific countries indicates that Pacific people's leadership processes are culturally driven. Having very few academic studies on leadership in the Pacific nations, the information provided in this section was drawn mostly from conference reports and individual experiences. Despite this information being non-academic, I believe it provides important information about the Pacific leadership and how it links to my participants' leadership experience.

\footnotetext{
${ }^{2}$ Pacific term for Pakeha (foreigners or non-Pacific people).
} 
The success of the leadership process in the Pacific nations is attributed to the collective efforts rather than individual. In documenting his speech to the participants in the Pacific Health Leadership Development Programme in New Zealand, Kavaliku (2006) reflected on the connection between culture and leadership. He describes culture as a way of living, and the belief that people's knowledge and understanding about their culture also influence their leadership thinking, experience, and the way they relate to each other. From his leadership experience in the Tongan Public Service, Kavaliku experienced the following leadership styles: tauhi vaha'a or respecting for others, creating trust among internal members within the organisation and key stakeholders, sharing responsibilities, and promoting ownership through persuading organisational members to understand that they own and are accountable for any work they do within a context (Kavaliku, 2006, pp. 5-11).

It becomes apparent from leadership experience such as this that the leadership processes of Pacific people are rooted in cultural values of respect, relationship building, and sharing. Kavaliku (2006) believes that the major problem regarding leadership experiences by most Pacific people in New Zealand is the uncertainties of the environment in relation to their culture. He urged the Pacific people to understand that their cultural leadership values are not unimportant in New Zealand but they need the time and confidence to adjust them into the environment.

Sanga and Walker's (2005) book Apem Moa [raise the bar] Solomon Islands Leadership, reflects on their understanding of leadership in the Solomon Islands and how leadership is applied in this particular context. They describe that leading by action is the most influential leadership process for the majority of Solomon people because people are judging leaders on their actions and achievements rather than their rank in the society. They said, "leaders in a Solomon Islands context have nothing to demand the support and cooperation of the people" (Sanga \& Walker, 2005, p. 31). This means that leadership in the 
Solomon Islands is driven by people working together, which highlights the fact that people are influenced by each others for the betterment of the whole.

In Tmetuchi's (1998) paper about the Palauan leadership which was presented to the Pacific Islands Political Studies Association in 1996, he shared cultural issues faced by Palauan people as an outcome of being governed under the United States' style of leadership. Tmetuchi described that when the American socio-political system was introduced in the Republican of Palau from 1944 to 1994, the key leadership challenge was related to ignorance of traditional leadership systems by the United State' administration. According to Tmetuchi, the traditional Palauan leadership system was much more collective oriented whereas the United States style focused on the individual system. He noted that "in the traditional system ... the stress was on harmony and working together. In the modern system, individual enterprise is admired" (Tmetuchi, 1998, p. 15). This quote describes that cultural behaviours of relationship building and working collectively are fundamental values underpinning the leadership processes of the Palauans.

While Sanga and Walker's (2005) book and Tmetuchi's (1998) paper do not deal with leadership in the public service, they are important in that they clarify how culture might influence the leadership process. The cultural influence was obvious when Tmetuchi showed the differences between the American and the Palauan style of leadership. The comparison Tmetuchi made about the leadership styles used by these two different countries draws attention to the interesting difference in leadership approaches and their link to the different backgrounds and experiences of leadership each group brought with them to the leadership process.

The above information about leadership in the Pacific nations is important because it clarifies the leadership potential of Pacific people which may be misunderstood by the majority of non-Pacific people interacting with them. 
Although my study is not focused on exploring the cultural complexities of the leadership process that my Pacific participants bring with them to their leadership experience in New Zealand, I hope that information collected in this study regarding participants' leadership experiences will raise awareness of what affects them as professional workers in non-Pacific organisations. The fundamental point to my study is the fact that any system or people intending to work with and for Pacific people must understand the collectively cultural focus of their leadership processes.

\section{Leadership process in the public service}

Literature on the importance and demands for leadership in public service organisations points to the need for a theoretical approach to social processes of collective influence, and to fully utilise human attributes and experiences (Alimo-Metcalfe \& Alban-Metcalfe, 2004; Parry \& Proctor, 2000; Terry, 1995). A review of public sector leadership in Australia, New Zealand, the United Kingdom, and the United States has revealed that both formal and informal leadership processes are crucial components of successful leadership. These leadership processes include participating collectively, communicating cohesively, creating trust, and relationship building.

However, much of the international research on leadership processes in the public service has dealt more with formal and informal processes gained from within the organisational setting than with leadership processes acquired from social interactions inside and outside the workplaces. There are several pieces of international research of significance to this study. They involve investigation of different leadership styles in public service organisations. This review section begins with literature available from international public service followed by local literature in the New Zealand Public Service. This structure helps to guide readers' understanding about the link between the leadership processes that are considered useful to success of both the international and local public service organisations. 
Towards the end of the 20 2 th century, Terry (1995) notes the importance of the sociological body of leadership literature for exercising bureaucratic leadership in America because it views leadership as a social process. According to Terry, socially bureaucratic based leadership is made up of two processes: the active interactions among members within the organisation, and the processes of social influence between the organisation's internal staff and external supporters. Terry also names the social processes of leadership as institutional integrity of leadership, emphasising what both individuals and organisations have contributed in terms of their distinctive collective life course.

Terry's (1995) research is relevant to my study in that it highlights that successful leadership considers the significance of acting cohesively towards achieving the organisational desired outcomes. The process of social cohesiveness demonstrates to people standards for how they are involved in the workplace, such as how they behave, and how they learn. Terry's study is also significant because it shows that the responsibility for cohesiveness lies with every member of the organisation, and effectiveness is achieved through their social cohesiveness in the leadership process. In that there is no difference between capable and less able leaders, everyone is a leader in their own right.

Loverd's (1997) research for his book Leadership for the Public Service Power and Policy in Action found that leadership in the United States' Public Service is power-oriented, based on formal positions. The question is where does power lie? In most organisations, as I experienced, the power lies with leading positions such as managers. However, Loverd states that the power of a leader can be easily lost if followers do not understand or interact well in the organisation's resources and strategies. Regardless of what leadership source or strategy a leader chooses, Loverd argues that careful attention is needed to building social interactions to help pull in power. 
Loverd's (1997) study is useful for understanding the ways in which leaders approach their followers, and also highlights the power of followers in the leadership processes. That is, if leaders make little attempt to fully utilise the leadership potential of their staff then the danger of distrust and demotivation may arise. In my view, power-oriented leadership does not create a place for members to express their actual perspective and experience of leadership. Generally, Loverd's research is central to the current study in that it shows that good leaders do actively make use of the power and leadership potential exhibited by subordinates.

In a study examining the effect of appropriate leadership actions in the Australian Public Service, Korac-Kakabadse and Korac-Kakabadse (1998) found the leadership process of communicating cohesively and consistency to have a favourable effect on the performance of the organisation. On the six organisations in which Korac-Kakabadse and Korac-Kakabadse were networking to measure the effect of leadership on organisations' performance, better quality of communication styles and high level of commitment at all levels were identified as contributing to a more amplified service orientation. They reported:

As the quality of dialogue and interactions improve, so does service delivery, partly due to the improvements of morale experienced by staff and management and partly due to the improvements in interfacing across the structure (Korac-Kakabadse \& Korac-Kakabadse, 1998, pp. 171-173).

It appears that communication is one of the leadership processes playing important roles in enriching the performance of public service organisations. As the key function of most public organisations is to provide services to the public while operating with strictly limited resources, Korac-Kakabadse and KoracKakabadse's findings essentially supported the use of more leadership processes for motivating people to achieve the organisational outcomes. 
In 2004, the Australian Public Service Commission launched a new leadership model known as the Integrated Leadership System (ILS) to develop leadership capability in the Australian Public Service (Podger, Simic, Halton, Shergold, \& Maher, 2004). In a comparative study of major public sector jurisdictions in the Australian Public Service, Podger and colleagues (2004) examined factors affecting the exercise of public administration. They found a need to improve leadership development and communications. Central to my study is the ILS view that leadership is at the heart of the organisational process, not an independent factor of the context.

Some scholars also argue that the way to achieve the public sector's multifaceted objectives is to increase and maintain people's effectiveness in the organisational leadership process (Alimo-Metcalfe \& Alban-Metcalfe, 2004). In their empirical study on the nature of transformation leadership in the United Kingdom (UK) Public Service, Alimo-Metcalfe and Alban-Metcalfe were concerned with the nature of leadership in the UK being dominated by the common understanding of leadership. These include being highly focused on white population leadership models, formal positions, and failing to consider that UK Public Service organisations are providing services to an increasingly multicultural population.

As a result, Alimo-Metcalfe and Alban-Metcalfe (2004) conducted various studies since 2001 of over two thousand managers and professionals from middle to top levels in the National Health Service and developed a new UK model of "nearby transformational leadership" (2004, p. 177). This model suggests that "leadership is fundamentally about engaging others as partners in developing and achieving the shared visions, and as such, it relates to distribute leadership" (Alimo-Metcalfe \& Alban-Metcalfe, 2004, p. 179). This model looks at leadership in the public service from another view, which is not just about managing staff and how best to meet their needs but more about creating an environment that gives diverse members some sense of belonging to the 
organisations. At this point, it is all about "connectedness" (Alimo-Metcalfe \& Alban-Metcalfe, 2004, p. 179) or strengthening relationship, sharing responsibilities, transforming, and developing each others' capacities for the benefits of the collective.

Much of the academic leadership literature available from the New Zealand Public Service is the work of Parry and Proctor. In their leadership survey with leaders of the public and private sector organisations in New Zealand, Parry and Proctor (2000) reported a need for more case studies in both private and public sectors to understand the appropriate organisational leadership processes in New Zealand. They suggested:

There is a need to maintain focus on the researching of leadership processes rather than leaders themselves. Because of the congruence of opinion about leadership as a social process, we need more understanding on what these processes are, and how they operate (Parry \& Proctor, 2000, p. 54).

It appears that the social aspect of leadership process is seen as the source of organisational success. That is, the role of people in the leadership process of any organisation is primarily to help the ordinary and extraordinary achievements of the organisation. Therefore, attempting to understand the types of significant leadership processes for people in the public service organisations is of major benefit to them and to the organisation's performance.

In a study carried out by Parry and Proctor (2001) in the New Zealand Public Service, which explores managers' perceptions on the characteristics of their organisational culture and immediate subordinates to promote leadership capacities. They found a range of issues which affect the leadership capability of staff members in public service organisations. These include transactional versus transformational culture, access to benefits and responsibilities for leadership development, and integrity within the public service. Although the study appeared to deal more with the organisational culture, and managers' integrity and leadership capabilities, Parry and Proctor (2001) provide valuable 
points in the final section of the paper by suggesting strategies for enhancing leadership in public service organisations. These include a need for shifting the organisational culture from working for individual goals to the collective goals of the organisation through sharing visions and communicating cohesively, promoting joint benefits and joint responsibilities of professional and career development between leaders and followers, and enhancing integrity (Parry \& Proctor, 2001, p. 9).

The above strategies by Parry and Proctor (2001) present an understanding about the nature of leadership process that may help to improve the challenges of working in a multi-cultural context such as New Zealand. Important to my study is what Parry and Proctor emphasise the need to consider leadership in the public service organisations as a collective responsibility. A later study by same authors also found that effectiveness of New Zealand Public Service managers is perceived as more transactional than transformational compared to the private sector (Parry \& Proctor-Thomson, 2003).

Parry and Proctor's (2001) findings are comparable with Snively and Roche's (2000) report on their survey of 52 Chief Executives in New Zealand Public Service organisations. Snively and Roche found that the hierarchical models of leadership in the public sector had influences the abilities of Chief Executives to make their organisations more responsive to change because of workers' resistance to control. Snively and Roche's (2000) report also found that Chief Executives desired a change in the leadership dynamics, from management to leadership, or from the traditional hierarchical focused to an approach that would provide greater empowerment to staff. This consistency of findings shows how research of leadership in the New Zealand Public Service can inform decision makers of leadership development appropriate for all levels. 
Studies of Pacific public servants in the New Zealand Public Service Little academic research exists on leadership issues relating to Pacific public servants in the New Zealand Public Service. The studies available are mainly government reports on Pacific staff progressions, as discussed in Chapter One of this thesis. The State Services Commission (2004) reported that the majority of Pacific workers are clustered in the production and service-related occupations. Although it is not the focus of my study to examine whether Pacific people are well or under-represented in leadership positions, I believe it is useful to reveal the problems they experience as a minority in New Zealand while they strive to succeed professionally. Having this focus in mind does not mean that Pacific public servants are less able to succeed to a leadership level. However, the data raise awareness about the different leadership experience they possess compared with their non-Pacific colleagues.

As noted in Chapter One, several attempts were undertaken by the New Zealand Government to address the issue of Pacific workers' poor representation in leadership positions. These included the Cabinet direction to implement a strategy for increasing the participation of Pacific public servants (SSC, 1993), conducting of Pacific Vision International Conference by the Ministry of Pacific Island Affairs (1999), a Career Progression and Development Survey carried out by the State Services Commission in 2000 (SSC, 2002), the State Services Commission special report on Pacific peoples in the New Zealand Public Service (SSC, 2004), and the Pacific public servants' leadership fono or public conference on Pathways to Leadership: Goal 2010 (MPIA, 2005). There is also a concern that if the above attempts are not understood and fully implemented by those who are involved, then issues regarding Pacific people in the public service may not be improved. The Ministry of Pacific Island Affairs (2006) reported:

... it is difficult to develop effective and comprehensive policies without direct involvement with the people whose responses, behaviour, and attitudes will ultimately make the policies work (MPIA, 2006, p. 17). 
It is apparent that the majority of the above studies on Pacific public servants issues centre on issues of concern to participation in leadership positions and policy making. Whilst these studies touch only generally on aspects of Pacific public servants' leadership capabilities, very little is known about the leadership process that they currently engaged in, in the New Zealand Public Service.

\section{Summary}

The review of literature related to my study indicates that leadership processes of Pacific public servants are still under-developed in the academic literature of leadership in the public service. As a result of this literature review, my study is expected to fill an important gap in the research of public servant Pacific populations. Despite various suggestions in government reports that Pacific public servants prefer recognition of their cultural leadership values as part of their professional skills in the organisation, little information is available regarding the leadership processes that Pacific people believe are important to their performance in New Zealand. Moreover, this research will be useful if it goes some way to compare the leadership process experiences of Pacific born and New Zealand born Pacific public servants.

The next chapter discusses the research strategies that I designed and employed for data collection and data analysis in the current study. 


\section{Chapter 3}

\section{RESEARCH STRATEGIES}

This chapter seeks to achieve the two purposes of methodological design mentioned by Denzin and Lincoln (2005a) in their in-depth study of qualitative research. The first is theoretically based and the other is practically based. Theoretically, I connect the theoretical philosophies of the project to the research strategies, and practically I provide a set of strategies for collecting and analysing data. To achieve the theoretical purposes, this chapter describes the research paradigms of this study, the research methodologies, and the research methods. This chapter also presents the outcome of the pilot study, and includes how practical aspects of the research strategies were attained for data collection and data analysis.

\section{The research question}

This study attempted to answer this research question: What are the leadership processes currently employed by Pacific public servants in the New Zealand Public Service? The following sub-questions were developed to assist collecting the answers to the research question, and further to the research objectives mentioned in Chapter One.

\section{Sub-questions:}

a) What do the Pacific participants believe are the leadership processes crucial to their work and organisational outcomes?

b) How does the Pacific public servants' cultural background affect their experience of leadership processes in the New Zealand context?

c) How does the organisational context affect the leadership process, as perceived by participants? 


\section{Research paradigms}

A paradigm is the set of basic theoretical beliefs usually held by researchers about the reality of the world. These beliefs guide their research processes in a way that will answer what they need to know about reality. Denzin and Lincoln (2005b, p. 183) defined a paradigm as a "basic set of beliefs that guide action". The selection of an appropriate paradigm relevant to employ in my study was fulfilled after studying the basic set of paradigms that generally underpin our knowledge of the world.

There are two main paradigms that generally underpin the knowledge of the world: the positivist and post-positivist paradigms. O'Leary (2004) distinguishes these paradigms from the assumptions that differentiate the world of quantitative and qualitative research methods. O'Leary describes that assumptions related to the positivist paradigm tend to be quantitatively oriented (or the world is scientifically driven), and assumptions related to the post-positivist paradigm are qualitatively focused (or the world is socially driven).

Thus, the knowledge in a positivist paradigm exists from the positive confirmation of theories through scientific methodologies and researchers conduct positivist studies to test theory (Cavana, Delahaye, \& Sekaran, 2000). Conversely, post-positivism asserts that knowledge about the world is constructed through human beings' experiences and interactions, and reality is not fixed because there are multiple realities out there in the world that may be explored (Denzin \& Lincoln, 2005a). Researchers conduct post-positivist studies to understand what is meaningful to human beings.

After examining the above basic paradigms, the post-positivist paradigm is the more appropriate paradigm for better comprehending the Pacific public servants' leadership process. Since this study argues that leadership is a process resulting from social interaction between members within a context, the 
subjectivist and constructivist assumptions of the post-positivist paradigm are central to this study because they claim the world to be socially constructed. According to O'Leary (2004), the subjectivist assumption is underpinned by peoples' experiences and meanings as the basis of factual knowledge, and the constructivist assumption accepts the importance of their interactions and interpretations in constructing meaning. This understanding of the world makes the study of human beings special because it indicates that the key to constructing reality is people (Patton, 2002). Guba and Lincoln (2005) state that the constructivist perspective is ontologically relative and epistemologically subjective. The ontological assumption argues that what can be known about the world depends on multiple realities, not on a scientific belief or experimental data. These multiple realities can be constructed by researcher(s) and respondent(s) as they interact and are involved in interpretations, according to the epistemological perspective.

Several reasons emerge to support the choice of the post-positivist paradigm. First, due to the lack of in-depth information regarding the Pacific leadership process, a combination of the subjectivist and constructivist beliefs in a postpositivist study may be appropriate to elevate a deeper understanding of the issue. Second, my involvement as a post-positivist researcher is relevant to identify what is meaningful to the individuals who participated in this study. Being involved with these subjects will allow me to explore the social meaning of the situation as it is understood by this group of individuals. The postpositivist approach is, therefore, the most appropriate paradigm for understanding the multiple realities about the Pacific public servants' leadership processes.

Last but not least, the assumptions of the post-positivist paradigm is fully supported by global leadership theorists such as Bratton and colleagues (2005) as an appropriate approach to understand the nature of leadership processes. Grint said “Of importance to the constructivist would be not what the leader or 
the context was really like, but the processes by which the phenomenon was constituted into a success or a failure" (1997, cited in Bratton, Grint, \& Nelson, 2005 , p. 22). Knowing that the constructivist assumption is one aspect of the post-positivist paradigm which describes the process of how people interact and engage with their interpretations to construct reality, Grint quote stresses this crucial point. In essence, Grint says that what is important for understanding leadership is to examine it as a process, not as a person or a style of the situation. Grint (2000) also says that the key difficulties for understanding leadership and improving people's skills of leadership is that most researchers have employed scientific analysis methods for studying leadership, whereas Grint believes that leadership is not accessible to scientific approaches.

\section{Research methodology}

Methodology is a research framework or a strategy of inquiry related to the set paradigm (Denzin \& Lincoln, 2005c; O'Leary, 2004). Since methodology is shaped by the set of paradigms used to conduct the research, my explorations of various methodologies have shown phenomenology to be an appropriate framework associated with the concept of the post-positivist paradigm and appropriate for the study of the Pacific leadership process. According to O'Leary (2004, p. 122) the world of phenomenology is "constructed and intersubjective". In other words, this means that the world is constructed through people's views and creativity, which is fundamentally grounded in peoples' interactions and cohesiveness. Phenomenology is highly dependent on the lived experiences of human beings (Creswell, 2007) and my study looks at the lived experiences of participants' leadership processes.

Several reasons emerge for my interest in phenomenology. Firstly, phenomenology is considered to be a strategy often suitable for student researchers to explore the lived experience of a phenomenon. It is also highly regarded by O'Leary (2004) for understanding the experiences of a particular phenomenon. In the current study, the phenomena refer to the leadership processes which are comprised of Pacific public servants' interpretations and 
experiences. Secondly, phenomenology is a useful methodology to understand and to explain the leadership process of Pacific public servants since little theoretical information about the leadership process of this particular group of people exists in a New Zealand context. Creswell (2007) said that phenomenology contributes to increased awareness about a phenomenon as experienced by several individuals. Essential to this study is the focus of phenomenology on examining how participants experience and perceive leadership rather than who they are and what position they hold.

Finally, phenomenology is suggested by O'Leary (2004) to be an appropriate methodology for investigating the complexities of the social world. From my experience, dealing with human beings is complicated because people are involved in a variety of different complexities such as biases, conflicts of response, and unresolved questions about social interactions. These complexities may ruin the leadership process and suppressed conflicts may end up creating scenarios that lead to conflict and tension. However, I believe that people are important because they are the key to success, no matter what policy is formulated and what technology is installed. In support, people are motivators in sustaining social interaction and in constructing meaning as they experience the world through inter-subjectivity (O'Leary, 2004).

Thus, since leadership involves people and people engage in various complexities, phenomenology is an appropriate methodology to understand those complexities of the people. For example, employing phenomenology to investigate participants' experiences may uncover complexities related to participants' leadership processes. This phenomenological view is compatible with the beliefs of the subjectivist and constructivist assumptions of postpositivist paradigm. 


\section{The research method}

Once the methodological approach is identified, the next step to consider is the research method appropriate for achieving my methodological approach. This study used a case study method. O'Leary (2004) describes the case study as a method employed to study the social elements of an individual, a group, or any other unit of social life organisation. In support, Stakes (2005) and Yin (2003) present the case study as a framework, and a form of social inquiry employed for the exploration of an issue through one or more cases within a setting. The concept of studying the social elements of a case is necessary to the present study, considering its emphasis of leadership as a social process.

\section{Case study research}

In the field of qualitative research, case studies enhance experiential knowledge of the case and the effects that contexts have on the case (Stake, 2005). The experiential knowledge highlights the information obtained from peoples' lived experiences resulting from their interaction with others as actual facts. This is the core of the current study, examining the practical elements of leadership as they are experienced by Pacific public servants. Moreover, one of the objectives of this study is to explore the effects of organisational context on Pacific public servants' leadership processes. Hence, employing case studies opens up multiple realities of leadership processes as experienced by Pacific public servants in a New Zealand context.

The case study approach was also chosen because of the limited nature of the thesis time-frame, and its scope means that it is appropriate to study a small number of cases in depth. The focus on a limited number of cases makes the study doable. Having chosen the case study as an appropriate method for studying phenomenology, the following sub-sections now talk about the nature of the case, the case selection criteria, and the actual participants. 


\section{Nature of the case}

There are three different types of case studies identified by Stake (2005): the intrinsic case study, the instrumental case study, and multiple case studies. According to Stake, an intrinsic case study is employed by a researcher who wishes to obtain a comprehensive understanding of a case of his/her interest. In contrast, the instrumental case study is used by a researcher who is interested in a particular issue and who then selects a particular case to provide an in-depth understanding of that issue. A multiple case study approach was then developed from the instrumental case study to gain a deeper understanding of an issue using a number of cases. Considering very little information is written about the Pacific leadership process in the New Zealand Public Service, the approach of multiple case studies was identified as an appropriate method to gain a comprehensive understanding of this issue.

\section{Case selection}

In this study, I decided to employ O'Leary's (2004) criteria of case selection. O'Leary suggests three steps in selecting an appropriate case study: define a case, decide on the number of cases to explore, and select a case to study. To define a case, it is significantly important to describe the different elements of the case that make it distinctive. The case in this study is defined as an organisation that belongs to the New Zealand Public Service department.

In deciding on the number of cases to explore, this study identified two cases. My research aims to examine the leadership process of Pacific public servants, as I believe that selecting different types of cases will provide different views and help to understand the research issue. Stake (2005) and Creswell (2007) support that having two or more case studies will provide in-depth theoretical analysis through comparison and contrast of the results. They also suggest that employing more than one case study will raise awareness of the complexities of leadership by providing multiple views, leading to a deeper understanding of the research issue. Therefore, the word case and organisation will be used interchangeably in this study. 
In selecting the case, this research identified two organisations that are well known in the Wellington region, particularly for their connection with Pacific peoples. The priority in choosing these two organisations comes from my personal interest in their experience and involvement in the Pacific public servants' leadership development. These two organisations also support the programme for development of leadership in the New Zealand Public Service, aiming to enhance and recognise the skills and influences of potential leaders. Both organisations are affiliated with the government departments and they are known in this study as the Mana and Motu organisations.

\section{Participant population, setting, and participant sample}

The target population is all the Pacific public servants in New Zealand and because it is impractical to speak to every single person of this population, the research setting of this study is the Wellington region. The Wellington region employs high proportions of Pacific public servants in fact, the second largest number in the country (SSC, 2005). In addition, most government departments that have large numbers of Pacific public servants have their headquarters in the Wellington region. For these reasons, I have chosen Wellington to be the key setting of this study.

A total of sixteen Pacific public servants participated in this study and eight participants were interviewed from each case organisation. All of these participants had more than one year's work experience in the chosen organisations. These people represented a range of levels of seniority and a range of functional ${ }^{3}$ areas within the two organisations. The majority of the participants' positions, thirteen of the sixteen, fell within the levels of Policy Analysts and Chief Executive Officer. Table 2 illustrates the breakdown of the participants' background information by organisation. Each participant in my study was given Tongan numbers as their alternative names. Those names have been used throughout this thesis for presentation and discussion of their data.

\footnotetext{
${ }^{3}$ Deputy Director-General, Chief Executive Officer, Deputies, Senior Policy Analysts, Policy Analysts, Finance Officer, and Project Officers.
} 
Using names was appropriate for ease of reference when analysing participants' data. Their places of birth will also be revealed in a bracket at the end of every quote.

\begin{tabular}{|l|l|l|}
\hline & \multicolumn{1}{|c|}{ Mana Organisation } & \multicolumn{1}{c|}{ Motu Organisation } \\
\hline Place of birth & $\begin{array}{l}\text { 6 Pacific nations-born and 2 New } \\
\text { Zealand born }\end{array}$ & $\begin{array}{l}\text { 1 Pacific nation-born and 7 New } \\
\text { Zealand born }\end{array}$ \\
\hline Gender & 2 female and 6 male & 7 female and 1 male \\
\hline Age & Range in age from 34 to 48 & Range in age from 23 to 37 \\
\hline Name & $\begin{array}{l}\text { Matelau, Taha, Ua, Tolu, Fa, } \\
\text { Nima, Ono, and Fitu }\end{array}$ & $\begin{array}{l}\text { Valu, Hiva, Hongofulu, Teau, } \\
\text { Afe, Mano, Kilu, and Miliona }\end{array}$ \\
\hline
\end{tabular}

Table 2: The background information of Pacific participants by organisation.

Table 2 illustrates interesting patterns from the participants' background information. The first pattern is related with the distribution of Pacific and New Zealand born. My study achieved a logical distribution of Pacific and New Zealand born in general, although an imbalance exists between the two organisations. The recruitment of seven Pacific nations born and nine New Zealand born was unexpected. Secondly, this study achieved a reasonable gender-balance although each organisation was dominated by either females or males, and while I did not expect to recruit nine female and seven male, I was pleased to have done so.

Thirdly, I was surprised to note the wide range of Pacific nations (Cook Island, Fiji, Niue, Samoa, and Tonga) represented, which achieves the original intention of recruiting participants from various Pacific ethnicities. In fact, the nations covered five of the six Pacific ethnic groups represented by the Pacific public servants in New Zealand. Finally, this study attained a fair distribution of age among participants from the two organisations, although Motu organisation was dominated by young people compared to Mana. It is important to understand that the majority of the eight participants from the Motu case were recruited from the Pacific Division of the organisation, and almost all of them self-identified as being Samoans. 


\section{Pilot study}

A pilot study is like a 'pilot of the boat,' (or researcher of a study) in terms of its role in finding ways through the waves (or difficulties) in the ocean (or field of research) to reach a destination or achieve the research goal. As a pilot of this boat, one of my primary tasks is to align the research strategies with what I need to know about the world, and employ a pilot study to test the consistency of the research strategies. This is to see if these research strategies were appropriate to collect relevant data from the field to answer the core research question and to achieve the research objectives. Hence, this pilot study was undertaken to improve the process of data collection as suggested by Yin (2003). This is supported further by Sampson (2004) who said that a pilot study in qualitative research is useful for refining research techniques but is also a significant approach to minimise the researcher's risks and resource wastage.

The pilot study was designed from the core research question and key subquestions mentioned above. These questions were developed from the literature and from my personal interest and interpretations. The participants in this pilot study were two public servants of Pacific descent who had been working in two different organisations in the New Zealand Public Service for more than one year. These participants, whom I knew personally and have had contact with through academic and cultural connections, were recruited by emails seeking their consent to participate in this pilot study. I recruited one female Pacific nation born public servant, and one male New Zealand born Pacific public servant to this pilot study.

This pilot study followed the formal procedures for conducting research during the field study. This included meeting all the ethical requirements of the university and ensuring the research information sheet and consent form reached the participants before the actual pilot study. Each participant was interviewed individually for about one and a half hours at their workplace. Their interviews were tape-recorded and later transcribed for analysis. Towards 
the end of each interview, participants' feedback on the design of the interview questions and how the interview was conducted were sought. Their feedback was useful for refining the process of the actual data collection.

The participants' responses in the preliminary findings indicated that their key leadership processes were driven by the organisations' key roles and from their experiences obtained from different cultural contexts. Both participants considered that their cultural background was an advantage to their work in New Zealand. However, some possible constraints of organisational contexts and cultural backgrounds were also noted to have an effect on their performance in a non-Pacific Public Service context.

During the interview process several interesting issues arose which are worthy of comment. Firstly, there were some interview questions which repeatedly addressed the same concept and this resulted in collection of similar findings. Secondly, I noticed that I interrupted the speaker during the interviews, and I took care to minimise this in the actual process in order to allow participants to finish what they were trying to say. Finally, since the focus of the overall research was still unclear at the time, it affected the design process of this pilot study and this was reflected in the findings. Taking into account the lessons learnt from the pilot study, I then carefully designed my data collection tools in the following section to be compatible with the core research question and objectives.

\section{Data collection tools}

This study collected mainly qualitative data using two main tools. The first tool was direct in-depth interviews and the second was the analysis of documents available publicly from the two organisations being studied. These tools were considered to provide rich information and to ensure consistency of the leadership processes experienced by Pacific public servants in New Zealand. The tools also link closely to the post-positivist paradigm of this study. O'Leary (2004) clarifies that the post-positivist paradigm is qualitatively research-typed, 
meaning that qualitative data considers participants' interpretations and meanings as factual information relevant to the research problem. This study, therefore, collected qualitative data from the Pacific public servants' experience of leadership processes. This section describes the data collection tools and how they were utilised in the study process. Issues of data analysis are covered in the next section.

\section{In-depth interview procedures}

In-depth interviews were chosen to seek a deeper understanding of leadership processes as experienced by Pacific public servants. One good reason for conducting interviews in qualitative research is that it will enable the researcher to reach the area of peoples' experience that usually remains unspoken, or the "inaccessible reality" (Perakyla, 2005, p. 869). Examples of these unspoken realities include people's interpretations, social interactions, and personal experiences. In my view, these inaccessible realities can be described as the 'invisible' reality of leadership as experienced by people in their everyday interactions. Experience is invisible because the reality of participant's interpretations, meanings, and personal experiences toward leadership as a social process are not usually seen and may not be revealed unless the researcher overcomes this gap by interviewing people who are involved. It is also important to understand that interview tools are appropriate research technique for uncovering realities experienced by Pacific participants because it associates with the 'Pacific Way' of oratory negotiation (Anae et al., 2001).

At the planning stage of this study, I intended to recruit participants primarily through non-random sampling methods such as the volunteer sampling methods (O'Leary, 2004). However, only five out of the total participants were recruited through the volunteer sampling method. I also attempted to recruit more participants through further invitation and direct contact with a few executive members from both organisations, but this was unsuccessful. In consultation with my Pacific Learning Adviser, attending several workshops for Pacific students, and learning from theses of past Pacific researchers, it was 
clear that the appropriate recruiting method for Pacific people was through their social networks with the researcher.

The recruitment method that did work to recruit more participants to the current study was through a snowball sampling method based on my personal networking. This was similar to the recruitment method in the pilot study. Due to my knowing very few Pacific people in the two case organisations, I contacted a few people whom I knew personally in the New Zealand Public Service. I had contact with them through academic and cultural connections and I sought their assistance for referring my research to their social contacts at the Mana and Motu organisations. These contacts included the two participants in the pilot study. Whilst these methods of sampling do not provide representativeness, they are appropriate for the selection criteria of participants because Pacific public servants are not easy to identify or access.

Participants' experiences of leadership processes were studied in one-on-one interviews where I was involved in asking them semi-structured questions. Since a semi-structured interview question is not fully fixed by the researcher nor completely free for participants' views, O'Leary (2004) considers that this type of interview question may be the best way to manage the interview process. An interview schedule (Appendix A) and an information sheet (Appendix B) were used to guide the interview process and sent out for participants to consider beforehand. This was aimed to keeping participants well informed and giving them opportunities to ask any questions they may have about the research. All communications from and to participants were done by phone and email. Some of the selected questions in the attached interview schedule came from Parry's (1998) suggestive questions for studying the leadership process. 
On the day of the interview, I provided a brief summary of the research objectives to the participants prior to the start of the interview, allowed them to ask questions, assured them that their personal information would be kept confidential, and gave them the consent form (Appendix C) to sign. During the interview, I started our conversation with some defined questions and then opened it up for participants' views. I began by asking the participants to reflect on any leadership memories they may have had and then continued according to the categories listed in the interview schedule. I used some follow-up questions to seek clarification and the questions were asked and answered according to the flow of conversation. At the end of the interview, I provided a debrief summary of the interview reflecting on participants' key contribution to the research. This reflection was important to acknowledge my participants' contribution and to ensure all questions were asked and answered.

Each interview was tape-recorded and conducted for about one and a half hours. Almost all interviews were carried out at the workplaces and one interview was conducted at a café. This was the participant's preferred venue; however, I found that the noise in the café from the music and people's conversation were quite disruptive. The noise affected the recording soundtrack and I needed more time than expected to transcribe this interview. Almost all interviews were completed in English and only one interview was conducted in the Tongan language. When each interview was completed, I transcribed it and then followed up any ambiguous data with the respective participant through emails. This was to ensure that the meaning captured was, indeed, the intended thoughts conveyed by them. The interview that was completed in Tongan was transcribed from Tongan to English.

\section{Document analysis procedure}

Document analysis is described by O'Leary (2004) as a tool used for collection, review, interrogation, and analysis of different types of documents. Such a data collection tool is essential to my study in several ways. The first is that documents helped to support my interview data. Researchers such as Perakyla 
(2005), Marshall and Rossman (2006) considered documents as another primary source of data to support any other types of data collection and theoretical works of a project. The second is that accessing data available from documents is practical and efficient because it can be carried out without people's involvement or any disruption to the organisation. Lastly, using document analysis in the current study was expected to provide in-depth background information about what and how leadership processes operate in the case organisational contexts.

There were various types of documents available for review. This study collected twelve published documents from both organisations. These documents included two statements of intent, two annual reports, five newsletters, and three policy papers. After a brief review of the sources available from organisations, the document analysis was then divided into two stages: before and after the actual data gathering. Prior to my actual data collection, I reviewed documents such as organisations' statement of intent and annual reports to increase my understanding about the organisations, what they do, and what leadership strategies exist. When the data collection was finished, I conducted a final analysis of newsletters and policy papers to confirm the ideas that emerged from the interviews. There was no formal way of conducting the document analysis in my study. I identified themes through repeated readings of the documents and recorded themes as they emerged.

While document analysis is considered to be an appropriate tool for understanding of a situation, there are two major limitations of document analysis worth mentioning. The first limitation is that the role of a researcher to collect, review, and analyse relevant documents can be limited by the availability of documents. The second is that the researcher can view the content of the documents from his or her own perspectives, making it biased to the researcher's views regardless of whether it is compatible with the authors' key points or not. 


\section{Data analysis tools}

There are two general purposes of data analysis central to this study. The first purpose is that analysing qualitative data provides an in-depth understanding of the phenomenon being studied (Cavana et al., 2000), or the leadership processes experienced by Pacific public servants. The second purpose is that the tools used to analyse data helped me as a researcher to provide relevant conclusions in relation to my key research question. Denscombe (2007) says that a qualitative data analysis tool allows different researchers to reach their conclusions. I started the data analysis during my data collection. Several themes emerged while I was collecting the data and recording them in my themes journal. In this section, I first discuss the method of qualitative data analysis that was used, and then detail the procedures for analysis.

\section{Thematic analysis}

When all the qualitative data were collected, it was then analysed using a nonnumerical analysis method called thematic analysis to identify key themes as they appeared repeatedly in the data. I used thematic analysis in my study to understand the meaning of the data. It was considered to be an appropriate type of data analysis for my study because it opens up the significance of findings developed from participants' interpretations and the document data. As Patton (2002) argues, thematic analysis is important for researchers to identify core meanings of the raw data.

The thematic analysis in this study was conducted in two levels: the analysis of individual cases, and the comparison of similarities and differences between the two organisations. Strauss and Corbin (1990, p. 204) refer to this as an "open coding" or the process of analysing data by breaking it down, comparing, and categorising it. Once the data were compiled and sorted, the analysis procedure then continued with the initial reading of every script. New themes emerged during the first reading and they were recorded using the summary column in Appendix D based on Tolich and Davidson's (1999) framework. I labelled emerging themes using coloured post-it notes. 
Further readings of my data continued to uncover and reveal themes through the exploration of key words and phrases, concepts, and metaphors, as they were used repeatedly by participants and appeared frequently in the documents. Patton (2002, p. 454) describes this as the "indigenous concepts" or the original ideas of data identified by key phrases, repeated words, and common ways that are special to the investigated participants in the context they operated.

After the summarising process, the thematic analysis of data continued using the second and third columns of Appendix D. This process identified the data that were qualified to be part of my findings by coding the summary data into preliminary categories. The most repeated themes were considered very important and were recorded under the positive coding column of Appendix D. The unclear data were then recorded under the negative coding column of Appendix D. The aim here was to have a deeper understanding of the raw data in response to the project's research question. Based on coding the data into preliminary categories, the final process organised the data into final categories.

\section{The process of final categorisation}

This section discusses the processes employed in this study to develop and construct the theme categories. The framework that was used to explain specific analytical actions associated with category development is Constas's Documentation Approach for category development (Constas, 1992). The primary value for utilising Contas's framework in this study is its potential to allow me as a researcher to provide explicit explanation of how and when the categories were developed. Constas developed this framework to encourage qualitative researchers to open up all aspects of their analysis process to the readers. Constas believes that the major problem with most analytical qualitative methods is that researchers do not provide readers with clear explanations of how specific categories were developed. Therefore, the welldefined nature of this qualitative framework and its processes was why it was considered useful to apply in this study. 
This framework develops the process of categorisation in two domains. The first domain is regarding the components of categorisation. The components of categorisation consist of three set of actions. They are origination, verification, and nomination. The details of these three components are described later in this section. The other domain is temporal designation, referring to the point during the research process where each category was identified, whether before, during, or after the actual process of data collection. Merging these two domains resulted in one documentation table as illustrated in Table 3 below. Each category in the table was assigned with a number from 1 to 8 as they emerged from the thematic analysis, and classified into two major themes: the organisational and cultural leadership processes. These categories are listed in the part of the table below the shaded area. The left-hand side of the shaded area indicates the components of categorisation and the right side of shaded area is the temporal designation. The key of data sources being used in this study is given in the bottom part of the table. They are participants' interviews and the organisational documents.

The components of categorisation comprise three procedural elements. One is origination or where the category came from. The second is verification, or how this category was justified, and the third is the nomination, or where the name of the category came from. The first component of categorisation is origination. Constas' framework consists of five loci of origination but only four were applicable in this study. They were the literature, programmes (or the objectives of the study), participants, and researcher's investigative perceptions. In my study, the category classified under literature was derived from a conclusion or a statement as the result of an existing literature review. For example, category 5 of Table 3, learning about leadership, was identified in the literature before the actual data collection and interview was the main data source for this category. In the case of programme evaluation, categories were derived from the research objectives. From Table 3, category 7 originated from the research objectives when the designing process was carried out before gathering data. 


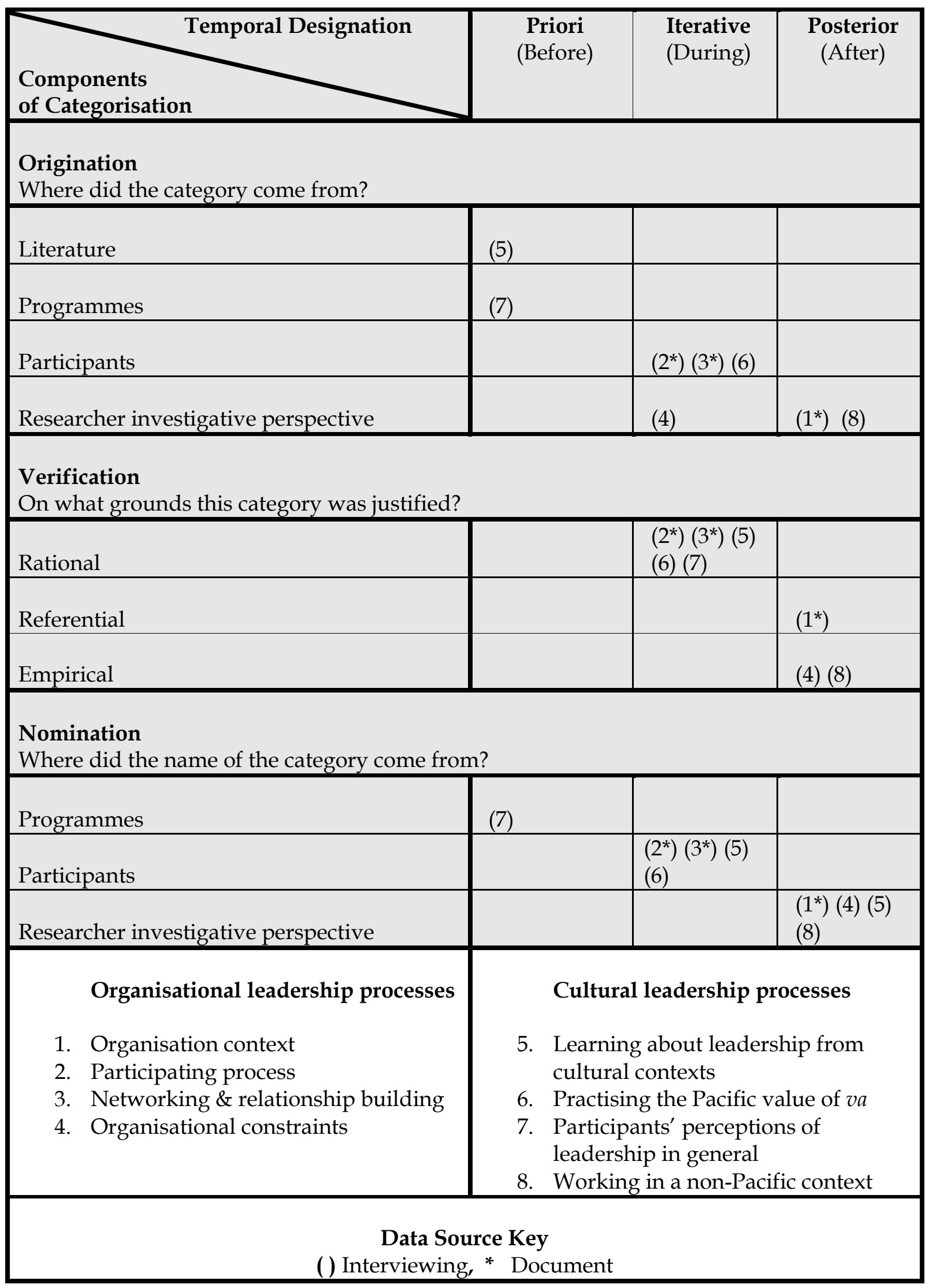

Table 3: Category development of Pacific participants' leadership processes, derived from Contas' Analysis Framework (Constas, 1992). 
Categories which originated from participants' viewpoints were identified under the participants' section, and categories identified from researcher's interests and perceptions were categorised under the researcher's investigative perspective. For instance, categories 2, 3, and 6 came from participants' perceptions during the interview process and were achieved from both interview and document data sources. On the other hand, categories 1, 4, and 8 were derived from my investigative perspectives as the researcher of this study, during and after the process of data collection. For example, category 4 was derived from my interpretation of interview data during the process, and categories 1 and 8 both originated from my analysis of interviews and documents data after the process.

The second component of categorisation was verification. This component describes the strategies used to justify any specific category. The Constas's framework consists of 6 original sources, but only three were appropriate to apply in my study; the rational, referential, and empirical approach to verification. The first approach to verification was rational, which involved depending on logic and reasoning. What is important here is that the categories should reflect some useful relationship and reasonable social connection. The majority of categories were derived from participants' social interactions with other members of the organisations. They were developed from participants' perceptions and supported in the organisations' documents. Thus, most of the categories (like 2, 3, 5, 6, and 7 of Table 3) were justified under the rational approach to verification during the process of data gathering.

The second strategy of verification is referential. This refers to the category that was justified using existing research findings and theoretical arguments as a point of reference. Category 1 was justified using the referential strategy by referring to existing literature of another author, after the process of data collection. The final strategy of verification is the empirical approach, using my own analysis of data without reference to other studies. Categories 4 and 8 were 
justified under this approach. The process of verifying these categories was carried out after the process of collecting interview data.

The final component of categorisation, nomination, describes the process of naming the categories. The process for nomination is similar to the origination process where names of categories may be developed from five original loci of origination. However, only three major sources of nomination were employed in this study to describe where the name of categories came from. They were programmes, participants, and researcher's investigative perspective. These three loci of nomination have been described above in the process of origination. The name for category 7 was developed from the programmes. This is when the research strategies were designed before the process of data collection and data for this category came solely from participants' interviews. Names for categories 2, 3, 5, and 6 were constructed from participants' data during the interview process. The name for other categories such as 1, 4, 5, and 8 were created from my investigative decisions as a researcher, after the data gathering. While the final decision for naming category 5 was developed from my analysis as a researcher, it was also noted that the concept of 'learning about leadership' often appeared in the participants' data during the process of data collection.

\section{Research credibility}

As discussed earlier in this chapter, the current research has been shaped by the underlying assumptions of the post-positivist paradigm in which people's interpretations and experiences constitute multiple realities of the social world. Knowing that issues of subjectivity and bias of findings can occur in almost all research, I handled those issues in various ways to ensure the credibility of my research.

The first action that I used to ensure credibility was that I conducted the whole process of this research, from the literature review to the final write-up in the guidance of the core research question. This is to ensure that the conclusion is 
consistent with what I need to know about the leadership process of my participants, and not influenced by any other means of conclusion. As O'Leary (2004) states, if the conclusion is drawn from outside the observable phenomena then it is not an indicator of a good research. To ensure clear relevance of analysis to the core research question and objectives of this study, findings were analysed in the light of the research question and linking findings to relevant leadership theories. I also tried to clearly report the participants' contribution and my view as a researcher in the presentation of results and discussions of findings.

The second action was concerned with the consistency of the research methods. To ensure the trustworthiness of data collected from my study, I employed various methods throughout the research process. These included using more than one data collection tool, using two case studies, recruiting participants from a reasonable gender-balanced and wide range of Pacific nations, and using participants' feedback to double-check the accuracy of their data. As O'Leary (2004) states, using triangulating data and findings add value to the authenticity of data.

Finally, I take full responsibility regarding ethical issues in the research process. One of my key roles as a researcher is to make sure that my study respects the confidentiality of participants' identity and their data by keeping all written data in a locked file and saved electronically using password protection. Ensuring the confidentiality of participants' data enabled them to trust me as the researcher and feeling comfortable to provide their views with confidence, as they understood that their data would be safely kept. O'Leary (2004) says that building good relationship and trust between the researcher and the participant is one way to ensure the credibility of the research. 


\section{Summary}

The exploration of research strategies identified the post-positivist paradigm and its assumptions as more appropriate philosophy for studying leadership processes experience by Pacific public servants. As it justified, this paradigm is useful to reveal the invisible and multiple realities experienced by people. In order to explore leadership process experiences by participants in the public service context, I decided to use qualitative case studies and employed two data collection tools. They were in-depth interviews and document analysis, and data collected were then analysed in a thematic analysis using Contas' categorisation framework to organise key themes emerged from the investigation.

After organising the data into categories, the findings were then presented as themes in response to the research question and the objectives of the current study. The results of the findings from the current study are presented in the next two chapters. 


\section{Chapter 4}

\section{FINDINGS}

\section{Themes that emerged from the case of Mana organisation}

The results of thematic analysis described in Chapter Three highlighted two categories of themes central to the current study: the organisational and cultural leadership processes. The organisational leadership processes were drawn mainly from what participants believed as key leadership activities in their everyday duties, and what factors they considered inhibiting their experience of these leadership activities in the organisation. The cultural leadership processes, on the other hand, were developed from participants' views on importance of their cultural background to their work, and difficulties they face while working in a non-Pacific workplace.

\section{The organisational leadership processes}

What follows are descriptions of themes related to the leadership processes in the Mana organisation. These themes comprise the organisation's context, participating process, networking and relationship building, and possible constraints of organisational context on participants' leadership processes at Mana. These organisational themes are now presented.

\section{The context of Mana organisation}

This theme describes how things are undertaken at Mana to achieve its core functions. I believed that providing relevant information about the background of the organisation will help readers in understanding the culture of the Mana organisation. It is also important to note that due to the confidentiality aspect of the current research, some of the inputs such as vision of the organisation and information regarding staff members are not discussed. Data collected from both interviews and document analysis identified several aspects of the Mana context. 
The first aspect of context is related with the organisation's processes. It is evident from the organisation's public documents and various government websites that Mana does not administer any legislation and regulations and hence does not publish external guidelines or manuals relating to any Acts or Regulations. Thus, Mana attempts to achieve its roles by formulating various policies, giving advice to government agencies, facilitating public meetings and consultations, and establishing other means of communication with the public.

The second aspect which contributes to an understanding of the organisational culture is the nature of power relationships at Mana. The findings revealed that the nature of power relationship at Mana is dominated by mainstream perspectives of the organisation as being hierarchical in structure. This means that the power for the overall operation of the organisation rests on the underlying legitimacy of the dominant government, as Matelau reported:

\begin{abstract}
Anyway in public service here in New Zealand, we are to serve one and only Minister. So if the Minister is happy with us and we strive to keep the Minister happy because he/she is accountable for the community [Matelau, Pacific nation born].
\end{abstract}

The above response indicates that what is important and how things have been done at Mana are driven by the government's expectations for the organisation through the direction of the appointed Minister. When the participants were asked about their views of the leadership processes at Mana, the majority of participants referred to their practice of the organisation's key roles as being driven by the Minister's expectations. Tolu said:

Our Minister drives a lot of our work. It is just in terms of the Minister's expectations and of course as an organisation it is our role and responsibility to brief our Minister. So we are accountable to the Minister and we have a sort of guiding document that we as an organisation have to do ... [Tolu, Pacific nation born]. 
It appears that Mana's operation is generally directed by the Minister's expectations as dictated in the organisation's guiding documents such as the statement of intent. In such a way, participants reported that managers and members of the organisation need to be involved accordingly in fulfilling the Minister's preferred outcomes for the organisation.

The final aspect of organisational context that is important in the leadership process is the way the organisation deals with the outside world. Mana engages in different activities such as meetings, conferences, and various events to build its relationships with the outside world for successful achievement of its key roles. Consequently, I was not surprised to learn from the participants that among the common leadership activities they performed at work were providing advice and facilitating meetings.

With information collected from interviews and the document data, it is evident that the context of Mana was formed by the government's priorities, the key roles of the organisation, and the organisation's relationships with external supporters. These have shaped how the Mana organisation operates.

\section{The participating processes}

This theme refers to the leadership processes that the participants took part in at Mana to achieve their key roles and to contribute further to the overall outcome of the organisation. When studying the interview data, I found common processes of participation. Most of the participants believed that the major advantage with the functioning structure of the organisation was its commitment to consensus in order to increase everybody's participation in achieving the organisation's overall goals. Nima said:

... the benefit is, everybody is involving, everybody has the chance to contribute or to work towards, you know, the outcome of the Minister [Nima, New Zealand born]. 
When I asked the participants about their views on daily activities which they considered as leadership activities at work, almost all participants reported being involved in various activities such as facilitating meetings, representing the organisation in outside meetings, and providing advice to the key stakeholders about their work for Pacific people. Participants' responses show that holding meetings with executives of other organisations and providing advice to the Minister and head of the organisation are key leadership activities which they were involved in for achieving the organisational outcomes. Participants also believed that those leadership processes were the driving force in guiding them to achieve their work programmes and the organisational outcomes.

Further to the above, participants also perceived that their participation in acting at higher positions when responsible officers were absent had encouraged them to feel responsible for the roles they carried out. Likewise, participants also believed that being given the freedom to establish themselves in the work they did perform allowed them to possess a sense of ownership of the duties for which they were responsible. Matelau reported:

I was working with someone who has given me freedom to do things. It does not mean that I can do anything that I want to do at anytime but I learnt how to take ownership in terms of the work. I think you rather have the courage, have the ownership when your boss gives it to you ... You will feel like you own that particular work and you are more responsible [Matelau, Pacific nation born].

As mentioned, participants perceived that the freedom at work is attributed to the people they worked with and should not be used for unnecessary things but specifically for their core duties. The exploration of the participating processes showed that the majority of participants often participated in the formal activities of the organisation. 


\section{The networking and relationship building}

This theme describes the ways in which participants were connected formally and informally in the organisation as opportunities to share ideas and information for the benefit of their work and for the organisation. Participants' responses to their social leadership processes and their key leadership processes at Mana indicated that they contributed collectively in different ways of networking and relationship building.

The first type of networking and relationship building employed by participants is their formal connectivity at Mana. In response to the questions about how they interacted with general staff members of the organisation, participants reported involving in weekly staff meetings and divisional meetings. The majority of participants reported that these meetings were conducted as a form of informing and reporting networking where everyone met to report and update the members of the organisation about their specific work programmes. However Taha explained that although these meetings provided a chance for staff members to raise concerns, it was not always the case because they had almost silent meetings every week. Taha said:

We have staff meeting every week, it is very quiet you know. Our Chief Executive Officer does a lot of talking and some of the middle managers. There is always a chance for staff to make comment but ... don't talk [Taha, New Zealand born]

When I followed up Taha's response about the reasons for staff being so quiet during staff meetings, she replied "there is some fear of being made a fool in front of the whole staff because our Chief Executive Officer can be very cut". What emerges here is that, among the formal leadership roles of a leader in an organisation like Mana are included controlling staff and managing decisions. Taha's response was supported in a later interview with $\mathrm{Fa}$, the leader of the Mana organisation. When Fa was asked to describe the ways he interacted with other staff members to achieve the organisational goals, Fa reported: 
People told me that I am too hard but I tend to set high expectation and to frighten people with the big stick and not enough of the incentives. I set high goals for myself ... I am aware that I am quite demanding and I do expect them out from staff [Fa, Pacific nation born].

It appears that the type of leadership strategy usually employed by a leader in the public service is controlling and putting high demand on staff. What $\mathrm{Fa}$ introduces above is that he uses the power of his position as a leader to manage staff, type of managerial instrument normally used by most formal leaders to control organisational processes. This result indicates that being a leader in the public service is a matter of complying with the requirements of the organisations, not of being a Pacific person or not.

The second process of networking and relationship building is the informal interaction between staff members. Almost all participants believed that the informal ways of interacting with other staff members were significant in motivating them. When their responses were followed up to describe their views about the ways of interactions they believed to be appropriate for staff members, they referred to their social informal nature of doing things. Fitu reported that informal networking at work is essential for successful outcomes.

I am thinking of the informal ways, not so much the meetings because you have meetings very often. Like the guy that I work with, I am trying to do it informally. I just walk over and have a chat with him and see what he is up to ... It is not even in your jobs description but they are still helpful [Fitu, Pacific nation born].

In response to the questions about the daily activities participants considered as leadership activities at Mana, sharing information and ideas were among the most repeated networking processes mentioned by participants. Taha shared with me how she interacted with her colleagues.

Oh I share it, I talk to them [her colleagues] ... and said to a colleague, can you have a look at this, do I sound too assertive for my boss? And when this colleague comes back and says no, I have not thought about it. But I also think that it is important that you have the support of people in the organisation [Taha, New Zealand born]. 
It appears that participants had the potential through a range of options to allow their colleagues to help them make appropriate choices for making proper decisions, and give them the ability to confidently exercise their collective decision making skills. This is similar to what Nima said, that he interacted collectively with colleagues through seeking each others' opinions, peer reviewing, and respecting the collective views in open discussions. Matelau also shared with me the way she created opportunities for her group members to collaborate on achieving the overall goals of the organisation.

I cut the documents into sections and divided the group into sub-groups and allow them to provide their comments and feedbacks within their own little group. And when we come to the meeting they will share what they have [Matelau, Pacific nation born].

The technique Matelau used allowed group members to employ various leadership skills such as providing their views right from the outset, giving them a chance to feel responsible for what they do, and thinking about the ways that might be appropriate to add value to the big picture of the organisation. Participants believed that these informal networking activities gave them confidence and created an environment where they could develop their own leadership skills.

The final process of participants' networking and relationship building is their involvement in building positive relationships between staff members within the organisation and their relationships with external supporters. With their relationships within the organisation, Fitu commented that the key for promoting good relationships with his colleagues was "to be honest and keep to your words". This indicates that creating an environment where staff members can interact with and trust each other would build good relationships between them and allow them to participate effectively at work. Similarly, Taha said that the "honest relationship" she utilised with her boss after an unexpected clash between them when she first started at work helped to heal their relationship. 
With regard to their external relationships, participants also said that maintaining effective relationships with other government agencies and Pacific communities was very helpful in encouraging them to implement the policies the organisation had formulated. Matelau described:

I make sure that we have a good relationship with other government agencies, as you may know from our Annual Report and Statement of Intent, we do not deliver any programmes. And we are relying heavily on other government agencies to implement our frameworks. In that way we have to have the good will of other government agencies to work with [Matelau, Pacific nation born].

It appears that Mana relies heavily on its relationship with other government agencies for the successful attainment of the organisation's core functions. It was also evident from the organisation's documents that Mana participates in various social activities with the communities to strengthen their good relationships. For instance, the organisation's newsletter for the months of March and April 2008 recorded that the organisation was involved in promoting the well-being of children on the National Children's Day in New Zealand.

Examining this theme highlights the fact that Mana holds the weekly meetings as the formal internal networking mechanism for staff members to report and discuss how best to do their work to achieve the desired organisational outcomes. However, the participants' responses indicated that informal networking also played an important role in their work. The informal networking and relationship building created by participants provided them with opportunities to work together with other members in the organisation. This is to share free advice and ideas for the benefit of everyone in attempting to achieve the organisation's goals. 


\section{The possible constraints on participants' organisational leadership processes}

This study also found some possible constraints on the organisational leadership processes mentioned above. These constraints are power, organisational structure, job complexities, and uncertainties relating to decision-making by executives regarding restructuring. In response to my questions about what was hard about the current decision-making processes at Mana, participants gave different answers. First, the majority of participants believed that political power and the expectations of the elected government through their Minister and head of Mana had influenced their leadership processes at work. Taha described:

Trying to meet the Minister's expectations and his [Mana CEO] are key factors that influence a lot in my works. Also the election government because if the election government will be changed and the structure of this organisation could be changed as well, who knows? [Taha, New Zealand born].

It is apparent that the power possessed by the Minister and the head of the organisation had an effect on participants' duties. This is a major concern for the participants because the elected government could be changed at any time, and when change occurs a new Minister may be appointed and the structure of the organisation would also be expected to change. This, according to most participants, has particularly affected the way they perform their tasks at work and how they interact with their peers and external supporters.

The second constraining factor to participants' leadership processes at Mana was in relation to organisational structure. The participants believed that the nature of leadership processes in a highly bureaucratic organisational structure like Mana limits the capabilities of staff members to create new ideas because they are systematically directed to perform according to their defined roles. Tolu commented that "it is quite hard to be innovative or creative because you had quite defined roles". Tolu's responses supported a point made by Fa regarding the problem with public service staff members' uncritical thinking, as he has found at Mana. Fa said: 
One of the real problems in the public service is that staff uncritically accept the view points that have been prompted by one ... and I annoyed that staff at this organisation has actually believe that because I don't believe it [Fa, Pacific nation born].

When I followed up Fa's response, he reported the third factor that other participants also believed to have constrained their leadership processes at Mana: the complexity of their jobs. Fa said that the nature of defined responsibilities, the uncertainties of urgency, and workloads were the problems because they limited sufficient room in the mind of staff members to provide critical reviews of a paper. He reported:

I think the people have been required to response to 400 policy papers that come and you know, when you read to response to 400 policy papers, you can't afford critical thinking. You just gonna have to respond to them as quickly so you can get them out of the door [Fa, Pacific nation born].

Fa's response indicates that the demanding nature of their work programme and uncertainty of urgency are high compared with the time available to complete those tasks. In that sense, participants believed that they barely had a chance to assess their work critically and to have a balanced life because of contradictions between their responsibilities to organisational interests and their personal needs.

The final factor was the uncertainty of decision-making related with restructuring of positions. At the time of this study, I was surprised to learn that Mana had just gone through a change process. The purpose of this change, according to the participants, was to improve the organisation's professional development where staff required set skills and qualifications in order to increase the capability of the organisation. This study found that the majority of participants' responses with regards to change indicated a huge uncertainty, especially on how decisions were made around the restructuring of peoples' positions such as directors' posts. 
From the results of examining the interview data, I theorised that the major issue that caused peoples' uncertainties about this change was centred on the uncertainties of decision-making related with restructuring of positions rather than the process of change in general. Most participants were shocked with the results of this change in terms of restructuring peoples' positions because they believed that people who left the organisation should not have left. According to the participants, the replacements for those who moved were unexpected and participants were still unsure how the new recruits would affect the organisation. When this new management were recruited, Taha reported missing the leadership activity of sharing ideas because the new recruits are working individually rather than collectively.

When I asked participants to describe some ways they believed could improve peoples' motivation during this time of uncertainty, they reported demanding that reconciliation should be carried out by leaders of this organisation with people who are still unsettled at Mana. Matelau said:

I think leaders should start seeing it now and making them [executives at Mana] believe that they gain the confidence of the staff ... and if people are in the dark not knowing what to expect or not knowing what is going to happen to their roles, and if they are not given clear directions right from the start, people will be very annoyed and frustrated and not very keen to work [Matelau, Pacific nation born].

The above response indicates the importance of providing people in the organisation with clear information about the restructuring of positions before the full implementation of the changes, which participants believed to be the major cause of peoples' unwillingness to work. Without doing so, these uncertainties of decision-making might create low motivation and might further influence their performance at work. 
From exploring this theme, I found two possible constraints of organisational leadership processes; one is an external factor and the other is an internal factor. Externally, it is obvious that the power of the elected government was beyond the participants' control. Internally, the organisational structure, complexities of participants' jobs, and the uncertainties of decisions made by decision-makers at Mana had constrained the types of leadership processes the participants employed. These limitations happened because participants believed that they were always required to perform beyond their set roles, but they had no control over the additional directions that were given to them.

While organisational leadership processes were considered critical to participants' set roles in the organisation, their cultural background was drawn on as a source of leadership strength underpinning the processes of leadership they currently employed at Mana. The next section describes themes of leadership processes associated with the participants' Pacific cultural backgrounds.

\section{The cultural leadership processes}

The cultural leadership processes include participants' leadership learning from a range of cultural contexts, practicing the Pacific value of respect, participants' perceptions of leadership in general, and working in a non-Pacific context.

\section{Learning about leadership from cultural contexts}

This theme describes how participants acquired learning about leadership from different cultural contexts such as families, churches, social connections in the communities, and workplaces. It was evident from the interviews that participants obtained leadership knowledge and skills mostly through informal learning experiences. This means that participants' understanding of leadership was not necessarily being taught but was learnt through observation, imitation, and action. 
When asked to describe their leadership background and in particular whether they had been taught about leadership, the majority reported "no" or "basically I have learnt from my experience" or "I did assume that as I go". It appears that most of them were not exposed to formal leadership training. When these answers were followed up about the ways they undertook leadership, almost all participants reported learning from their cultural contexts. They gave different answers.

The first and dominant cultural context of leadership learning that participants often referred to is their home-based leadership skills. Taha told me that her parents' leadership skills of being polite and very disciplined trickled down to her, as she believed it guided her in how she related herself to other people. Ono recalled learning from his grandfather's model of collective leadership. He described his grandfather as a hero from his ability to get along with people and make provisions for the benefits of the collective.

The second cultural context for participants' leadership learning is from their participation at church services and other social activities. Almost every participant reported employing the leadership skills they attained from their church ministers and religious beliefs, as they believed it played an important role in the way they did leadership. Ua told me that his religious belief gave him patience in dealing with the complexities of his job. It is therefore apparent that participants considered God as a leader to them in their work, as they mentioned praying to God for guidance and help in their decisions at work. Other participants described learning about leadership from their participation in social group activities, as they believed that the lessons learnt from those involvements were useful to their understanding of doing their best at the workplace for the benefit of the collective. 
Finally, other participants expressed their own learning experiences. Ono talked about learning from working in the public services in his home country. He believed that the quality of work obtained from the people he worked with and professional opportunities available for him there were valuable to his work at Mana because so far he had not had access to any training opportunities at this organisation. Nima reported that his leadership learning was influenced by where he spent his formative years despite being born in New Zealand. He described that he learnt from his time in a Pacific nation especially around youth participation and being given the chance to experience leadership by actually doing it.

Not all the participants acquired leadership learning informally. A few did have formal leadership training. Two, both Pacific nations born, out of the eight participants obtained leadership learning from the courses they took at university and from leadership courses they were involved in. However, it appears that while those formal ways of learning are useful, participants believed they were not enough to gather leadership in the public service and in the communities. This is part of my conversation with one of these two participants.

Me:

In what ways they are not enough?

Fa: $\quad \quad \quad \quad$. because leadership in the community is about you as a person, your cultural knowledge and awareness, the language you use, the relationships you have with other people, how helpful you are to young people ... you are not a leader unless you use the right language and be respectful. You can only be a leader if people refer to you as a leader ... I can be a leader as a manager in the public service because I know the laws, I know the standards, I know how to manage budget, I know how to recruit staff but that is not enough ... [Fa, Pacific nation born].

Although Fa did not describe the reasons for not considering his professional leadership training as being taught, Fa described obtaining leadership knowledge mostly from informal learning such as watching, listening, and copying. It appears from Fa's response that the types of leadership needed for 
an organisational context is different from that experienced informally in a social community context. In the public service, according to $\mathrm{Fa}$, leadership process is structural whereas leadership process outside the public service context is not structured but socially constructed, depending on how leaders present themselves to others. What emerges from analysing the above conversations is the need to give attention to the importance of leadership as a social process in the organisation contexts. It may not be the leadership focus in many organisations but it is important to know that informal learning in the organisation always exists through peoples' interactions and their view of a particular situation.

In response to my question about their views on the importance of the informal leadership learning experiences from different cultural contexts to their current work at Mana, participants' responses revealed two common values of importance. The first value that they got out of this informal learning was confidence. Participants believed that if they did not have those informal learning experiences they would find working in the public service more difficult. The second value was that participants described it as helping them understand the qualities of a leader. Most participants believed that the qualities a leader should have are: the capabilities to collaborate with people of different cultures and personalities, to make decisions based on collective views, and to move people together towards common goals.

Further to participants' views of learning about leadership is their perception of leadership support useful for them in New Zealand. My study found that most participants did not have formal leadership activities in the public service and it was a concern from the participants. Fa reported:

Not a lot and it has been one of the areas of concern ... I don't believe there is been enough in the way of opportunities or trainings or resources to enable and to support further development of Pacific public servants [Fa, Pacific Nation born]. 
When I questioned participants about the most helpful leadership support for Pacific public servants, most participants desired mentoring. When asked to give an example of whom they would best receive help from, they indicated that they would like to receive help from Pacific people at a management level in their organisation or in any other organisations in the New Zealand Public Service. They felt that such people would be easiest to seek advice from as they would provide relevant guidance for people from the same ethnic group. A few participants also added that in a New Zealand context those mentoring support systems can be better equipped with academic studies like management and leadership courses.

When asked to provide the content they might wish to include in a leadership workshop, some participants reported wishing to include the values of Pacific leadership in terms of respect and service. They believed this emphasis would provide a better understanding of peoples' cultural identity. Fitu wanted to demonstrate leadership. What emerges from his response below is the fact that leadership is a process and should be disseminated through allowing people to experience it rather than being told. Fitu said:

I think maybe I won't run a workshop. I will probably do it differently. Maybe it could be a workshop, but I will put them in a situation where they might demonstrate you know actions ... actions-based because to me that is what leadership is, it's not about standing in the front and telling you this is how you do it, you know, it comes from within and people need to practise that [Fitu, Pacific nation born].

The exploration of this theme found two types of participants' learning experiences: one is informal learning from cultural contexts and the other is formal learning from professional training. Whilst participants' formal learning experience plays a significant role in their leadership processes, it appeared to be insufficient to guide them in their work in the public service. So, what is considered important to participants' effective leadership processes at Mana is their informal leadership learning. 


\section{Practising the Pacific value of respect}

This theme emerged from interviews and was compatible with the organisation's values of respect to illustrate the types of action that participants believed to be most helpful for enhancing peoples' motivation in the workplace. This notion of respect was commonly described and understood by participants as one of their cultural values that controls how they present themselves to other people in order to make a difference in their behaviour.

When I asked participants about their views of how they encouraged people around them to feel motivated, participants gave different views on practising respect. The first is considering other peoples' views. Almost every participant believed that the Pacific cultural knowledge of respect gave them the understanding of the need to be respectful of others in terms of their opinions. Despite being born in New Zealand, Taha told me that her parents' advice and the way they brought her up to be humble taught her about the concept of respecting other's views and not being "fiapoko" [being smart or people who are thinking they know everything]. This is to make sure that she is exhibiting respectful behaviour. The element of respect needed to empower colleagues in their work is given in the statement below by Matelau.

... respect should be there, I am not talking about being very submissive and adornment where people run over you but the respect in a way where you respect other person's point of view and maybe to agree and disagree to some extent ... and there is time that you can't always win and I think it is important that you know why you can't go down that way [Matelau, Pacific Nation born].

Although it appears that respecting others' views can be a problem because it might result in a win-loss situation where one side may be agreeing and the other side may not, participants insisted that respecting other peoples' views brings happiness and happiness brings success. Ua commented that respecting other peoples' views brings happiness leading to empowering them in whatever work is required out of them. 
The second way of practising respect is treating other people in the way they wanted to be treated in the organisation. Almost all participants described treating others well is a useful action for encouraging people's motivation, as they believed that people would like to be treated as they wish. Nima reported "I treat them in the way that I would like to be treated" and more importantly is to understand how people feel and attempt to build a close relationship with them when their feelings are hurt, as he believed that in some cases people are demotivated by non-work-related matters.

The final way of practicing respect, according to the participants, was to demonstrate through actions. Most participants believed that demonstrations of leadership had contributed to building trust in people and influenced others to produce significant change in their performance. Taha described her leadership actions of timeliness and punctuality as her key leadership actions that she used to show a good example for others, as she believed that "actions speak louder than words".

The exploration of this theme, practising the Pacific value of respect, found that participants respected other people through taking into account their views, caring for people by treating them as they would want to be treated, and being a good model to others through their actions. This therefore confirmed that participants' unique understanding of their cultural background has helped guide them in how to relate with other people in their workplace more effectively.

\section{Participants' perceptions of leadership in general}

The majority of participants conceptualised leadership as a social action, and quality leaders are those who lead by good action. Here are representative answers from the participants:

Leadership to me is about serving ... [Taha, New Zealand born] Leadership is creating trust ... [Nima, New Zealand born] Leadership is basically providing direction... [Matelau, Pacific nation born] I think leadership is about being able to share ... [Fa, Pacific nation born] 
The above responses show that leadership is about acting and interacting reflecting on how people behave. Actions such as serving, creating trust, providing direction, and sharing are all examples of social processes, as experienced by participants to influence others. These definitions also highlight the leadership processes currently employed by participants at Mana. Each of the above definitions contributes significantly to understanding the values of participants' leadership processes. For example, leadership as a service reflects the importance of the participating process, creating trust highlights networking and relationship building, providing direction give special attention to the value of learning about leadership, and sharing represent respect.

The other participants perceived leadership as the attributes of people doing leadership. According to the participants, the attributes of good leaders include i) someone who understands their peoples' feelings, thinking, and work, ii) someone who is strong enough to take criticism for what they do as leaders, and iii) someone who has transformational leadership qualities. Tolu described a transformational leader as someone who is not directing, but guiding others in the way they think and allowing them to partake in the decision making to build confidence in them.

When participants were asked to give evidence of their leadership ideas at Mana, most of them reported exercising them in their everyday work in this organisation. For example, Taha described applying the idea of leadership as the collective action of sharing information and ideas with her colleagues. Ua also reported demonstrating his leadership notion at Mana through his participation in team activities in order to complete what they have planned to achieve. 
In response to my question about who should be involved in doing leadership, almost all participants believed that the potential for doing leadership exists in all people but is exhibited differently in different contexts. According to participants, leadership in a family context can involve everyone in the family whether you are mother or a father, as everyone contributes to the betterment of the family in their own right. In an organisation, participants believed that leadership includes members from all levels because they all contribute in some way to achieving the organisational goals. Moreover, participants believed that leadership is necessary for movement and change as it gives direction and guidance for people in terms of how to achieve what they are trying to achieve.

Whilst leadership is important, participants insisted that it was missing from Mana in certain ways. In general, more than half of the participants described wanting greater feedback from immediate superiors on their performance, as they believed that providing feedback was an important leadership role. The participants felt that the importance of feedback was to help them understand their weaknesses and strengths and to adjust their performance based on those weaknesses. However, having regular feedback from their bosses was not happening according to the participants. Other participants also mentioned that their organisation lacked important leadership action of recognising peoples' successes. The participants at Mana were particularly concerned about the recognition of the good work done by those who had left the organisation in the change process. Taha said:

If it change management then it should be changed and fixed it ... we don't have to cut people and leave it like that because these people came on board for some years [Taha, New Zealand born].

It appears that a lack of recognising people's values and a lack of considering the social significance of people are the major issues that Mana may need work on to improve ways for handling change in the future. 
When participants were asked to describe the types of people they wished to work with to do their jobs better, nearly everyone's answers were common in two respects. The first type of people is those with positive attitudes or the type of people that participants believed to have good relationship with others, who were able to work in a team, and who had an understanding of what they do.

Nelson Mandela was the most famous example mentioned by several participants. Of particular importance were his influential skills to touch the heart of many people. Taha described Nelson Mandela's influential actions of being a shepherd with his sheep as a good model for leadership. Fa said that although Mandela was not a rich man, his actions to communicate a vision and enabling processes and strategies to develop benefits for the community as a whole were very much an influence on his people. In my view, the participants described Mandela as a good leader because of his ability to persuade the people to produce significant change in their work through his good guidance, negotiation, hard work, and understanding of his people and others too.

Other participants wished to work with people from the same ethnic group and people who would understand them. Tolu said that he "feels better to work with other Pacific public servants". Working with people who would understand them was also important. Nima said that "having a better understanding of where Pacific public servants come from or their thinking" help to develop a good relationship between them and their colleagues. This indicates the fact that participants' understanding of leadership is socially and culturally driven in the New Zealand workplaces.

\section{Working in a non-Pacific context}

This theme describes the participants' views about the influences of their cultural background on their work in New Zealand. In response to my question about their views on how their cultural background affects their actions and participation at work in New Zealand, seven of the eight participants insisted that their cultural background was an advantage to them. Fitu said that his 
understanding of the Pacific context had helped him to provide effective advice to key stakeholders in New Zealand about Pacific history and culture. Tolu reported that the values of Pacific culture and beliefs such as collective team work contributed a lot to his work in New Zealand.

When participants were asked about how being of Pacific descent affected their leadership experience in New Zealand, nearly every participant believed that being of Pacific descent benefited them in the work they did at Mana. Despite being born in New Zealand, Nima described that being of Pacific descent gave him the confidence to perform his job for the benefit of the collective. Similarly, Fa reported that he felt responsible for the Pacific community because he was of Pacific descent. However, this was not the case for all the participants. Fitu believed that his success at work in New Zealand was not reliant on being of Pacific descent or not, but rather on individual skill and adaptability to the environment. He said:

I think it's about how adaptable you are to the environment. Again just focus in the skills on what you are doing [Fitu, Pacific Nation born].

Whilst participants' cultural backgrounds were considered as strengths to their work in New Zealand, they also shared with me some of the challenges they had experienced. The major difficulty was attempting to merge the needs of participants' Pacific culture and non-Pacific culture because of a conflict of understandings and expectations between the two cultures. According to the participants, this difference happened because the Pacific leadership is collectively based, whereas leadership in a non-Pacific context is individually focused. Participants felt that most Palangi people preferred to work individually whereas Pacific people loved to work and discuss things as a group. The other major hardship they faced in New Zealand is a lack of understanding diverse leadership among the Pacific nations by Palangi people. Most participants believed mismatch of perspectives could create differences of understanding, as this New Zealand born participant said: 
I can have really good conversation with my non-Pacific colleagues but when we talk about work its different story because she [her Palangi boss] brings a different perspective and I bring a different perspective and then she wants to push her perspectives or what I think is incorrect from a Pacific perspective [Taha, New Zealand born].

Despite different views about working with Palangi people, Fa, a Pacific born participant believed that working with them could contribute to outcomes of Pacific people because the quality of knowledge and skills they possessed would contribute to the successful outcome of the organisation.

The results of examining participants' perceptions about working in New Zealand indicated the significant contribution of their cultural background and being of Pacific descent to their experiences of leadership. Their views about the ways to practise collectively and the cultural knowledge they have had shaped the way they employed leadership processes in New Zealand. However, the challenges they faced in New Zealand was a concern for them because the participants faced constraints on their abilities to employ their cultural leadership processes effectively in New Zealand.

\section{Summary}

In general, the eight themes presented under the two headings of organisational and cultural leadership processes showed the understanding of participants' leadership processes. The key leadership processes experienced by participants at Mana were the participating, networking and relationship building, learning about leadership from cultural contexts, and practising the Pacific value of respect. What emerged from the Mana case is that participants' formal and informal experiences of leadership processes are significant to their performance at Mana. However, participants' informal leadership experience was more useful for them in New Zealand.

The findings from the Mana case also indicated two factors constraining participants' leadership processes. The first factor relates with the organisational contexts. The majority of participants reported being trapped 
within a highly systematically organisational structure which is controlled by power of the Minister and executive members. This, according to the participants, limited their potential to create innovation and critical thinking in their leadership experience. The second factor is related to the cultural background of Pacific participants. While the Pacific values underpinned the participants' leadership processes at Mana, it was evident that their cultural leadership processes were constrained by mismatches of understanding the 'Pacific Traditional Ways of Leadership' between the Palangi and Pacific people.

A theoretical discussion of the Mana case will follow in Chapter 6 of this thesis. The next chapter presents the themes that have emerged from studying the leadership processes of the Motu case. 


\section{Chapter 5}

\section{FINDINGS}

\section{Themes that emerged from the case of Motu organisation}

The description of themes in the Motu case is similar to the structure of Mana and they are discussed under the two categories of themes; the organisational and cultural leadership processes. The discussion starts with the presentation of participants' organisational leadership processes.

\section{The organisational leadership processes}

Themes related to the leadership processes in the Motu organisation are similar to the Mana case but described differently. They include the context of the organisation, participating processes, networking and relationship building, and possible constraints affecting participants' leadership processes. These themes are now presented.

\section{The context of the Motu organisation}

Providing the background information of Motu, where participants worked, is an essential grounding for increasing readers' awareness about the organisation's context. Similar to Mana, some of the organisation's strategies such as vision and information related to staff members are not discussed due to confidential issues. The understanding of different aspects relating to the Motu context emerged from the interview and document analysis data. In the Annual Report for the year ended 30 June 2007, it was noted that due to highly significant key service role of Motu to the community, the organisation administers legislation and regulations.

The Motu organisation is based on a bureaucratic structure where it operates under the umbrella of elected government and appointed Ministers. Annually, the Minister and Head of the organisation work together to finalise the strategic priorities and performance objectives for Motu as set out in the statement of intent. Hence, Motu reports quarterly to the Minister on the overall performance 
of the organisation. When participants were asked about their views on how decisions about what to do and how to do things are made at Motu, the majority reported that a lot of decisions are made by the Minister and those who possess formal responsibility to make decisions. Hongofulu said:

Well a lot of our decisions are made by our Minister. It is just the nature of working in the public sector that often the whole organisation or your team or your group is moving in one sort of way [Hongofulu, Pacific nation born].

Kilu gave a similar response. As one of the leaders at Motu, Kilu described that the structure for making decisions at Motu comes from "my Minister and my boss". Having this leadership structure in mind, she mentioned keeping good relationships between her and her Minister and boss. From an operational point of view, Miliona said that her boss made most of the decisions and only referred to her if the decisions concerned any part of her jobs.

It appears that what and how things are done at Motu is driven by government priorities. Hence, the organisational processes such as strategies, policies, and structure are aligned with government expectations. In such a way, Motu employs a hierarchical structure to control the organisation key activities. This is illustrated by most participants saying that the power for decision-making rests on the Minister and executive members of the organisation.

\section{The participating processes}

Despite the understanding that the power for making general decision at Motu belongs to the Minister and executive members, my study found some common views of participating in the process of decision-making. The first aspect of the participating process is considering the contribution of the collective in the organisational leadership activities.

In response to my question about their daily leadership activities at Motu, almost all participants reported engaging in giving advice and support to the Minister and other government agencies, providing advice to the co-workers and committee members about Pacific issues, and ensuring that the organisation 
provides quality services to the community. It appears that participants' involvement in providing service is one of their key leadership actions. They believed that providing quality services to the community is a leadership action because they are working toward an outcome.

Secondly, other participants believed that the advantage of the decision-making process at Motu is that everyone gets a chance to participate based on their formal accountabilities. Kilu believed that the process of decision making at the Motu organisation is much more collaborative in engaging people from all levels throughout the organisation. Similarly, Afe reported liking the organisation efforts in ensuring the participation of the collective in decision-making process. While this leadership process considers the collective views, at the end of her response Afe highlights the necessity to maintain the participation of the collective in the process. She commented:

In this organisation they are getting better in participation across the board. Chief Executive Officer has an open door policy but on a professional level he is a hard man ... he is not always available and decision-making needs to happen right across the board [Afe, New Zealand born].

This response indicates that staff members' participation in the process of decision-making should be fostered at all levels, as the leader of the organisation may not be always present to finalise every decision. While the Chief Executive Officer's "open door policy" may be an advantage for staff members to feel comfortable meeting him and be able to voice opinions, his professional role for controlling staff members could be the main reason of him being "a hard man". It appears that although the majority of the participants concurred on the fact that considering the collective in the participation process is of benefit to them and the organisation, conflict and bias are challenges of this leadership process. As Teau said, participating in the process of decision making in the organisation is hard compared to a family context because not everyone may agree on certain decisions. 
The final aspect of the participating process is allowing people to be involved in the process of decision-making in any form of responsibilities they carry out at work, as participants believed that it is a worthwhile opportunity to give people a sense of belonging to the organisation. Mano said:

It's really good, I think being able to have some saying over projects that you work on or type of work that you do. Whether it is sitting at your desk and writing a lot or being able to research something or being able to work in a group. You know, having flexibility and some controls over that are so important. It's one aspect of feeling value at your workplace [Mano, New Zealand born].

It appears from Mano's response that every single person in the organisation does have potential to participate in the leadership process regardless of what role they perform. The basis is allowing them to grow in the organisation by recognising the leadership skill and knowledge they bring with them to their job, and applying those valuable contributions in the organisational processes. The leadership process of participating was evidenced at Motu through delegation and allowing staff members to participate in various decision-making mechanisms. Valu described how he allowed his colleagues at work to participate and experience leadership activities. He said:

I sort of delegated the leadership to him [his colleague], so give him a chance to become a leader. Now he is a leader... At first he was not confident and rejected the offer because he felt so inferior to the job. So what I did I broke down the works into small activities and start with simple ones and when I felt confidence with him, I then asked him to do the whole work [Valu, New Zealand born].

What Valu introduces above is the fact that everyone is capable of doing leadership but they should be given the chance to experience it. He did this by allowing his colleague to take the opportunity and participate, giving his colleague appropriate guidance to keep him on track in what he is trying to achieve, and delegating the responsibility when he had the confidence. Hence, Valu emphasises different types of leadership processes: including participating, sharing, respecting, and inspiring processed. Valu's leadership action above is paralleled to Kilu's experience when she said that she allowed people to participate in the leadership process by providing them with clear and honest 
information. Kilu believed that such an approach motivates people to create their own thinking and come up with their own decisions based on the information they have in their hands.

\section{The networking and relationship building}

Participants' views and the organisation's document data showed a number of formal and informal networking and relationship building activities at Motu. The document analysis confirmed that the formal process of networking and relationship building is one of the organisation's key leadership roles, although this was not manifested in the interview data of this case.

The first formal process of networking and relationship building is collaborating within the organisational processes. The Annual Report for the year ended June 2007 stated that Motu made connections in many discussions and services development with internal stakeholders to assist with scoping the basis for the organisation's strategic long-term plan. In such a way, the organisation offers a range of Ministerial support services for the Ministers of Motu to keep active formal relationship, with internal stakeholders. Likewise, Valu reported that the organisation's internal policy of working from home is building trust and good relationships between them and their managers. He said:

One of my arrangements that I have is ... that I'm able to work from home on a Friday, so they give me a longer week-end. That is around building trust on my manager, that when I go home I actually do the work [Valu, New Zealand born].

The second formal process is working together with other government agencies. To maintain its good relationship with external supporters, the organisational documents reported that Motu was often involved in facilitating inter-sectoral activities and projects with other government agencies to discuss and link its key roles with the government priorities. While formal networking and relationship building can benefit the organisation in general, Hongofulu believed it is hard in terms of accessing and adapting to different types of people. She said: 
For a more formal relationship ... it is just killing yourself to be able to get someone attention or access. So you need to rely on different things. It's all about being adaptable to how other people works [Hongofulu, Pacific nation born].

It appears that formal networking and relationship building is not necessarily the only way to motivate participants. In fact, participants' interviews confirmed that their informal networking and relationship building such as sharing free ideas also plays an important role in inspiring them to succeed in their work. Almost all participants believed that it helps in encouraging them to perform their best at the workplace, as Hongofulu reported:

I think the relationships that you have with other people are important part of achieving your vision because that is your relationship with others that will ultimately help you achieve that vision. I think that is the most importance thing I have [Hongofulu, Pacific nation born].

In the Motu case, several strategies of informal networking and relationship building were identified mainly from participants' interview data. The first informal networking process that was highly emphasised by participants is sharing free ideas and information. When the participants were asked to describe the way they interacted with their colleagues at work, almost all participants reported sharing knowledge and experience with general staff members for the benefits of the collective. Providing mentoring assistance to new recruits and less experienced staff members were among the most repeated leadership processes mentioned by the participants because they believed it is important to keeping them well connected and focused. Teau described:

... supporting someone who is new to the organisation or is kind of less experience. And you know that is a home experience driven thing. I mean, you are sharing your own experience, you are passing on skills to them but you are also supporting them in a personal way to help them feel comfortable and safe at the workplace [Teau, New Zealand born].

What is central from Teau's response is the way she applies her home-based understanding of leadership such as sharing experience and caring to support new staff. Respectively, Valu reported sharing his knowledge and helping his 
colleagues at work. He believed it creates mutual trust and good relationship between him and other staff members, although it is not part of his official responsibilities. Valu reported:

I am trying to be transparent. So if I know something which I think that it might be relevant to you, I will pass it on to you ... So we have got that whole way of sharing and trusting for our inputs... Sometimes we go for a coffee and socialise, you know that, but I think it's back to the point I said about asking for help. When someone is asking for help, I help them ... they are really happy that I have actually helping them and it is also about that whole relationship building [Valu, New Zealand born].

The second informal networking and relationship building process employed by participants is how they communicate with other staff members. The majority of participants believed that their abilities to have clear communication with colleagues were necessary to understand each other's needs and expectations. Kilu commented that her informal communication skills contributed to the process of guiding people "because no system or process is enough on its own to guide people to what they need to do". In that sense, participants believed that providing clear communication is the leadership skill that a leader should have. To give people enough direction, enabling them to feel confidence in doing whatever their leaders are requesting from them, and more importantly, to have an active networking system in order to foster relationship building.

Likewise, from a leader's point of view, Kilu described utilising her informal communication tools for contact with staff, as she believed they contributed to good networking, understanding people expectations, and building confidence. Kilu said that her personal contact with staff members was the appropriate means of communication to build good relationships among them. She also believed that knowing her staff personally added value to networking and relationship building. Kilu reported: 
The first key day to day activity is really being visible and being seen. I am not a manager who sits in my office all day. You know, I get out and see people face-toface. If I get something that I need to feedback on I don't serious just wait until I see them again. I will go to see them ... meet everybody and know what all people in my group and really connect with them personally. Its also help the set of accountability that people at least likely to try to do something if they know you personally. [Kilu, New Zealand born].

In my view, Kilu's response is useful for understanding the process of networking and relationship building because she stresses the importance of personal communication in the organisation. She believed this keeps staff members well connected and motivated them to be effective at the workplace. Kilu also shows some sense of respecting, caring, and considering the value of her staff by attempting to have time to meet and follow-up with them on important work-related matters.

Finally, participants wished to work in their own comfort zones in New Zealand which led them to the establishment of their social networking at Motu. Participants believed that establishing an informal Pacific social network helped to strengthen their relationships and willingness to work in a non-Pacific environment. They agreed that this good informal networking at the Motu organisation empowered them to seek each other out and support for each other. Hongofulu reported:

The strength of the Pacific group in this organisation is that all the Pacific members have great networking and peer skills and they are great in maintaining their relationships [Hongofulu, Pacific nation born].

The exploration of this theme found both formal and informal leadership processes of networking and relationship building very useful to motivate participants to succeed. However, participants considered their informal experience of networking and relationship building more useful to them because it strengthens friendship building, team working, and understanding of people which is one of the leadership skills emphasised by most participants. 


\section{The possible constraints on participants' organisational leadership processes}

The organisational constraints at Motu are similar to those at Mana, but slightly different in nature. There were two group of factors identified as having constraints on participants' organisational leadership processes. One is the external factor of government power, and the other is the internal factor of the uncertainties of change and job complexities.

With questions relating to participants' leadership processes in the organisation, almost all participants referred, firstly, to the influence of external power in their leadership processes at Motu. Participants said that a lot of decisions are made by the Minister, as they believed this to be the nature of working in the public sector. Hongofulu said that it is a "political process" that should be understood by every member although it may contradict their view. This is because the power of the Minister and the organisation's executives can affect staff members' positions and may lead to job losses when positional restructure is undertaken, according to Hongofulu. Hongofulu said, "your work could be cut short or changed by the Minister". It appears that the dominant power within decision making in this public service organisation belongs to the Minister. Hence, participants said that staff members had little control over their work regardless of how much effort they have contributed to a particular job because of the power wielded by the Minister and his/her executives.

The second is the internal constraint of the uncertainties of decision-making related with restructuring. Although Motu was undergoing change at the time of the current study, participants at Motu did not have as much influence on this change as those at Mana. This was a concern for participants, particularly because they saw that their colleagues' motivation had been influenced negatively by this change. For instance, Mano reported struggling with her unsettled colleagues at work, especially those who had served the organisation for a long time. When participants were asked to give their views of how best to deal with the uncertainties of change, they reported allowing everyone to view their feelings about change regardless of what position they hold. Hiva reported: 
Communicate the changes that are going on, give everybody regardless of what they do in the organisation or what their position is. A chance to voice how they are feeling about it [change] and also what they think should happen. I think giving people the opportunity to input and seeing the organisation as whole rather than a layer at the top [Hiva, Pacific nation born].

When participants were asked to give their views on the type of people who could possibly help the organisation to get through this change, participants reported wishing to have people who can articulate a vision for the organisation and are able to communicate that to people. They believed it would be easier to deal with change if the vision is explicit. For instance, Kilu described that managing change is one of the leaders' key roles in the public service, but they should do it in a way that could help to implement change and at the same time encouraging people to work.

From exploring this theme, the results indicate that participants' organisational leadership processes are influenced by both external and internal factors at Motu. Therefore, their leadership potential can be limited to the power they hold in their respective roles. The next section presents the participants' cultural leadership processes.

\section{The cultural leadership processes}

This section presents participants' cultural leadership processes of learning about leadership from different cultural contexts, practising the Pacific value of $v a$, participants' view on conceptualising leadership, and working in a non-Pacific context. These themes are presented below.

\section{Learning about leadership from cultural contexts}

As in the Mana case, this theme describes how participants learnt about leadership from a range of cultural contexts such as families and their social connections in the communities. When participants were asked to reflect on leadership memories, almost all of them reflected on the leadership style of family members. Participants believed that observing and imitating their family members' leadership actions were the starting point to their leadership learning, and preparing them with confidence in the organisation. Hongofulu reported: 
It is given me expectations of what I want from someone that is going to lead in a work environment. It is a difficult question because you get into I think the ways that leadership is different in different settings. For me the leadership examples that I have had in my family and as a child have sort of influenced me in a way that I may conducted myself in life and work [Hongofulu, Pacific nation born].

When participants were asked to describe their leadership background and in particular if they have been taught about doing leadership, five out of the eight participants said they had no leadership training. The majority, however, acquired leadership skill and knowledge informally from different cultural contexts. The most repeated cultural context for their leadership learning was from their family members. Afe reported learning about serving from her parents, as she believed this to be an important leadership skill for keeping family members well connected. She said that serving others is a responsibility that she learnt as she grew up and which was freely given by her parents based on what came from their hearts. Valu described learning about acknowledging mistakes from his grandfather. He insisted that this is an important leadership skill that a leader should possess to build trust in people which he found was missing from most leaders he had known.

Kilu reported that although it is challenging working with people at different levels in the organisation, the leadership skills she learnt informally from family were useful for making decisions for the benefit of the collective, not just for a particular group of people. She reported:

The lesson I have learnt about how you make hard decisions in environments where people might have different views ... And I think that is an important impact for me, learning from all those family leaders. Then all those leaders for me are made decisions that did not look like they were the right things but actually were important. And they were the decisions that have made for the benefit of the collective [Kilu, New Zealand born].

Participants' means of learning about leadership was dominated by informal learning from their cultural contexts. Generally, only one of the eight participants was exposed to formal leadership training in the New Zealand Public Service and two of the eight participants considered learning from their university studies. 
Kilu reported being involved in the leadership programme for Executive Masters in Public Administration, which she believed gave her leadership skills and knowledge appropriate for her duties at Motu. However, Kilu insisted that she learned more about leadership from her informal networks.

I had formal trainings but I think a lot of my learning has actually coming from observations and experience [Kilu, New Zealand born].

Towards the end of participants' interview, they were asked to comment on leadership support available for them at Motu and leadership support appropriate for them in the New Zealand Public Service. In response, almost all participants indicated lack of leadership support available for them in the organisation. It was noted from participants' data that there is one leadership programme available in this organisation specifically for Pacific people. However, participating is based on certain requirements and applicants must meet those selection criteria in order for them to be able to attend this programme. Due to lack of leadership support available for them at Motu, Miliona reported making use of informal networks as a form of learning strategy outside the normal working hours.

We meet in our time over coffee and lunch, we share each other what experiences, what hardship that you are going through ... There is no leadership programme that can gather up for those kind of things, you know, they don't have things like that [Miliona, New Zealand born].

When questioned about the most helpful leadership support for them, most participants reported mentoring. The type of people participants wished to receive mentoring from is Pacific people from leadership level in the New Zealand Public Sector and from older Pacific people, as participants believed they are appropriate people to provide leadership support relevant for Pacific public servants in New Zealand. They wished to have different types of mentoring such as cultural mentoring, a self-developed mentoring, and a leadership programme that can explore the quality of Pacific leadership and its benefit to the organisation strategies. 
Participants also concurred on the fact that different ethnicities have different values of leadership, and there is necessity in the public service organisations to consider those values in their human development strategies. Knowing New Zealand is a multi-cultural country and an increasing number of skilled migrants, most participants believed that decision makers should consider diverse values in the organisation strategies. Afe reported:

I would like to see some mentoring, leadership programme, exploring how can you really fully be who you are in mainstream and drawn who you are as a Pacific to strengthen the organisation but what I find is that mainstream is not prepared for that. ... I also think that because of the demographic of this community and the growth of diverse businesses in New Zealand, you need to be able to work with different culture and to be willing to engage with people who have different values, willing to learn, willing to build your capacity. I think also, people that believe that professional development of the organisation includes cultural competencies [Afe, New Zealand born].

From exploring participants' learning about leadership, this study found that formal and informal learning experiences are both useful, but it was evident that participants' informal learning experiences were more important. As Afe said "learning from the book is one thing but learning from actually doing it is another thing". It is therefore apparent that participants' cultural context is the major teacher for their leadership learning mainly through informal experience. This way of learning, according to the participants, had contributed significantly to maintain their social connectivity within family members, social groups, and colleagues at workplaces. This theme therefore indicates that learning in an organisation is a continuous process that comes from people's behaviours as a result of their informal experience.

\section{Practising the Pacific value of $v a$}

This theme was developed from participants' data to explain the unique contribution of their cultural value of $v a$ to their leadership processes at Motu. The word $v a$ is normally understood as respecting others. However, the word $v a$ in my study is slightly different from the European word 'respect'. I describe va as 'allowing the potential of each person', emphasising the importance of allowing yourself to develop your own skills in the context you are working in. 
This term, $v a$, is used as the title of this theme in order to cluster the shared importance of respecting as emphasised by participants.

In response to the question of how their cultural background affects their actions and participation at work, the majority of participants reported applying $v a$ at the workplace. Afe described applying $v a$ in everyday communication with other staff members allowing them to express their views and to learn from each other, as Afe believed that acknowledging others' views is fostering motivation. Afe reported:

... my cultural knowledge of the va. I apply it with my colleagues I give you space to be able to know who you are, to be able to communicate with me, and I am giving you that respect I hope that you won't subrogate that ... So I do apply certain values and principles of what leadership for everyday communications from my upbringing in the workplace ... again it is all about respect [Afe, New Zealand born].

It appears that the leadership process of $v a$ is more than just the normal respect we experience in life. In everyday life, respecting others may refer to giving someone special admiration or consideration because of his/her position in the society. However, Afe's point of $v a$ is slightly different and it gives special attention to diversity, emphasising the importance of caring for the leadership skill and knowledge that different people bring to the organisation as an outcome of their cultural background. As Afe described, va refers to the process of allowing your colleagues to grow in the organisation by giving them a space to actually display their own leadership capabilities.

In my view, Afe tries to emphasise that va is caring, listening, and believing in the potential of every member in the organisation regardless of where they come from and what position hey hold in the organisation. As a possible English translation, va can be thought of as space for development. It describes the continuous process of allowing people in the organisation an opportunity to exist and develop in their own competencies. Central to this study is the usefulness of $v a$ to effective communication in the non-Pacific context, and the importance to 
keep it as an ongoing leadership process. While the majority of participants were using $v a$ for their interactions with colleagues at Motu, there was also a necessity to emphasise $v a$ in the public service leadership. Afe went on and said:

"... love is one of the things that determine our wellbeing, our happiness, our being able to work, our being able to have good relationship, our being able to be good people in the society, but very hard for people to talk about ... I think caring and loving for each other, the respect for each other is missing in organisation that I am in [Afe, New Zealand born].

It appears that leadership in the public service organisations places great emphasis on the economic wellbeing of the organisations, and fails to understand how people feel which is believed to be a crucial factor for a successful organisation. I believe that this lack of emphasis on the development of social capital may be related to the results-oriented nature and the set priorities of public service organisations.

From a perspective of $v a$ viewing it as human respect, Hongofulu described employing $v a$ in her everyday duties at Motu, as she believed that human respect had enriched her understanding about the people she looked up to as leaders of the organisation and the way she presented herself to others. Hongofulu described $v a$ as "fa'aaloalo" which is another Pacific term for respecting others. She said:

It does culturally. It comes from like, there is some Samoan principles like Fa'aaloalo it is just like respect and Tautua which is service ... That is something, you know, that is fundamental to me from young age. Respect your seniors and respect your elders ... Yeah it's always present [Hongofulu, New Zealand born].

Although Hongofulu was born in New Zealand, her culturally based understanding of leadership is still very much influenced by her upbringing in a non-Pacific context. This response indicates that being of Pacific descent has benefited her a lot in her work in New Zealand, as she believed that the Pacific leadership values of respect and service are always presence and worthwhile applying at Motu. 
Likewise, $v a$ is important in the process of decision making at Motu as the nature of making decisions at work is similar to that of the participants' cultural background. From my Pacific point of view, decision about big events such as marriage and usage of land at home must follow the traditional way of discussions and approval. In the case of marriage, the final decision on events and the programmes are not reliant on the wedding couples' decisions but upon agreement between members of the two families through various discussions and meetings. Kilu reported utilising such a leadership process at work.

... my personal experience helps me think about things at work is the fact that we [public servants] don't make decisions by ourselves. Those important decisions go through a long process of discussion and debates. So you know, if you wanna use land at home, for example, and so you are going to be married. You know, there are all branches of your family that have a say in how that marriage should happen. And I think that process of dialoguing and talking is quite a good skill [Kilu, New Zealand born].

The above shows that the notion of $v a$ incorporates respecting the collective view and considering the fact that individual members within the organisation cannot make decisions on their own. From my experience, making decisions at almost all public sector organisations such as Motu have to go through a long process and there is a high dependency on people at different levels to produce effective decisions on an issue. While the Pacific value of $v a$ is important for participants' leadership processes at Motu, few participants reported experiencing difficulties in applying $v a$ in New Zealand. Hiva mentioned that learning to question aggressively in the organisation is not matching well with her culture belief because of respect but she believed that having such a communication skill is imperative to her effective performance at Motu.

Exploring participants' responses on $v a$ indicated that the Pacific value of respect advantaged almost all participants at Motu because it gave them significant understanding of how to relate themselves to others, and to consider other peoples' views in the process of decision-making. 


\section{Participants' perceptions of leadership in general}

Participants understanding of leadership was conceptualised in two ways; one was around the idea of leadership as social actions, and the second related to the qualities of people doing leadership. More than half of the participants described leadership as something to do, as Hiva described "I realised it wasn't about who knows you and for me it was more actions oriented". Participants often repeated the practical aspect of leadership such as "serving others, helping others, motivating others, and considering those who you served". This is compatible with the dynamic processes of leadership as "two-way flows", emphasised by Hongofulu. She described leadership as a dynamic process of relationship between the leader and the people. Her response below indicates that leadership is a continuous dynamic social process where the leader has influenced on, and is influenced by people. She clarifies her meanings for this two-ways flow of leadership as follows:

I think it is getting away from the idea that a leader is who they are and they influence the group. Also works in the other direction, that the group influences what kind of leaders they have and makes the leader or defines the leader in a way as well. It's like a group or a group of individuals' perceptions of their leader influences, the type of leadership, and the type of leader that you have ... it is not a one-way thing that the leader projects themselves on to the group and who it is and what they do [Hongofulu, Pacific nation born].

It appears that leadership is not a one-way process where a leader takes control over the people they lead but it is a set of actions dependant upon leader's and people's influences on each other and by each other. When I asked Hongofulu to describe if this idea of leadership was evidenced at Motu, she reported "not so much", as she saw different styles of leadership at Motu and believed that "it is probably the styles of leadership they [leaders] employed". She did not explain these different styles of leadership.

In the second part of conceptualising leadership, participants described leadership as the qualities of a leader. Valu described his idea of leadership as being able to lead and having the quality to lead people. When he was asked to clarify his meaning of quality, he reported "being respectful, honest, trust, and having a vision". Bringing these qualities together with other participants' views 
make up a list of qualities of a leader: i) having a strong sense of communication skills, ii) a humble person with values of respect, honest, and trust, iii) selfmotivated, and iv) being able to make sacrifices.

The majority of participants believed that leadership is important for guiding people towards achieving common goals, ensuring the involvement of the collective in the decision-making process, and for a productive organisation. Miliona said that "leadership is really the key in helping everyone move in the same direction and holding groups of people together". Miliona's response shows that leadership is necessary for moving towards a shared desired outcome.

Missing leadership from Motu was also a concern from the participants, which they described in various ways. First, participants experienced lacking the support of their cultural driven style of leadership such as support from an older Pacific person in teaching them the ways to cope as a minority in the mainstream organisation, and the support for their social needs. Hiva said that two and three days of bereavement leave are not enough because the Pacific bereavement process needs require a longer time. Second, participants missed the social development of people such as emotional development, as they believed it contributes towards happiness in people, motivating people to work, and leading to productive service. For example, Afe reported that "love" is one of the values that determine the wellbeing of the organisation but it is a "no-no situation" in the public service. Finally, participants believed that in Motu, recognition of success was lacking and this was a concern they wished to develop in the organisation.

Regarding the type of people to be involved in leadership, participants believed that everyone has the potential to be involved in certain ways and in different contexts. This highlights the fact that leadership is a collective involvement. For example, in the public service context, participants believed that everyone should be involved because everyone has a role to play to achieve the overall outcome of the organisation. 
Exploring participants' views on leadership in general shows that they are common in viewing leadership as a social process of collective influence within a context. In response to the questions about their critical leadership actions and their critical social processes, participants' perceptions were common in three major types of influencing skills: communications, influential actions, and culturally relationship-based. It also appears that leading by action is the most influential action in the leadership process, as Valu said "if you want to change people from here to here, they want to see actions now". Among the most repeated phrases that were identified to describe the process of influential actions were; lead from behind, walk-the-talk, and work behind the scene. When participants were asked to clarify these statements, their responses were common in viewing them as actions-based personalities that people can influence others and by others through ethical actions.

\section{Working in a non-Pacific context}

This theme describes the effects of Pacific culture on participants' leadership processes in New Zealand. When the participants were asked to describe their views about working in New Zealand, the majority of participants reported benefiting from their cultural background. Their cultural understanding of leadership as relationship and collective influence was considered useful, as they believed that their culturally relationship-based leadership process in the family is a useful tool to make connection with other staff members of the organisation. In particular, about how they present themselves to others regardless of whether they were born in the Pacific or not, Hongofulu said:

... relationships are important with everyone regardless of whether they are Pacific or non-Pacific ... with our [Pacific] culture we place a high value on it [relationship] in the way that you interact with people, how you conduct yourself around people. That is important in my work [Hongofulu, Pacific nation born].

Similar to Hongofulu is what Kilu emphasised as a social-relational leadership process. Kilu learns how to manage the complexities she faced in dealing with different people in the organisation by using the relationship and communication skills she gained from being part of a big family. Kilu reported: 
I have got a pretty good handle on the complexity of different branches of the organisation, how we work across ... from being part of a big extended family ... and we all need to talk to each other about what is going on in the family and it is a strong sense. So I think some of that, what we could call probably that social and relational capital on how that can make decisions more effective [Kilu, New Zealand born].

While participants' cultural background advantaged them in their working in New Zealand, they also experienced some difficulties of having their cultural value in New Zealand. Working with non-Pacific people was one of the areas of concern for most participants. Only three (two New Zealand born and one Pacific born) out of the eight participants mentioned having no problems working with their non-Pacific colleagues. The others believed it is good working with Palangi people but have experienced some conflicts of view and understanding. The mismatching of participants' leadership experience with that of their Palangi colleagues was one concern. Many participants believed that the leadership style of most Palangi people is very much self-oriented which is all about their individual value on how best to climb the ladder to the top positions. However, participants reported that the nature of leadership for Pacific people is collectively relationship-based, placing greater emphasis on the communal than individuals' efforts. Teau reported:

For example, a responsibility is to get the views of everybody. That is a very Pacific thing as well because it is also collective thing as well, it's not so much on individual ... Well we have gained a Pacific team, we have shared values and ... it's quite a bit easier to get the collective, sharing and responsibility for things, but it is a little bit harder outside of the square of our own Pacific team, I think yeah [Teau, New Zealand born].

When I followed up her response with a question about why it was hard, she reported having difficulties with the mainstream timeframes set up to deliver her key roles at work because it mismatches with her Pacific timeframe. It appears that participants bring with them some valuable leadership experiences which may not match with the leadership style of the dominant culture. 
The other constraint of bringing participants' cultural values of leadership to New Zealand is lack of understanding of the leadership diversity that exists among the Pacific nations. This conflict of understanding was described by Valu as an "uphill-battle", believing it is a never-ending battle of misunderstanding. When I followed up Valu's response, he reported being displeased with the way Palangi people grouped Pacific people together assuming one solution fits all, as he believed that one solution cannot gather the diversity that exists among the Pacific nations. Afe says that misunderstanding between the Pacific and Palangi people is a "never ending process ... at the end of the day they [Palangi] never know what it feels like to be a person who is a minority". Attempting to learn about the Pacific value of leadership may not be an important interest for most Palangi people. However, these findings suggest necessity for an environment which values diverse leadership skills.

"Tokenism" was another constraint experienced by participants while working in New Zealand. Most participants believed that the public service key role to improve the outcomes of Pacific people in New Zealand is less effective. For example, Hiva and Teau reported that the key problem at Motu is that a lot of the Pacific framework is not implemented by the entire organisation. Although it is one of Motu's ultimate goals, participants believed that the Pacific public servants seem isolated trying to implement those frameworks while the entire organisation continues implementing their own policy and failing to consider the successful implementation of the Pacific frameworks. Hiva reported:

I think one of the weaknesses in this particular organisation is that there is a lot of tokenism for Pacific ... The Ministry of Pacific Island Affairs have developed a Pacific Analysis Framework and I see it finally enough, no one else in this Ministry seems to use it but it has been part of how we do our work in the branch [Hiva, New Zealand born]. 
What emerges from the above responses is a demand for decision and policy makers to monitor and ensure effective implementation of the Pacific frameworks in the entire public service organisations. Participants believed if these frameworks had been implemented successfully, they would be able to see some change now. Despite these differences about working in New Zealand, several participants believed that it is not about being a Pacific but about the skills and reality of being in any public service jobs.

\section{Summary}

The results of findings from this case found four key leadership process experiences by participants of Motu. They are the participating processes, networking and relationship building, learning about leadership from cultural contexts, and practising the Pacific value of $v a$. However, the findings from this case showed that participants' abilities to employ these leadership processes effectively in New Zealand were constrained by the organisational contexts and cultural differences.

Participants' cultural backgrounds had shaped their leadership processes at Motu, although various difficulties were reported. Participants felt comfortable working with and being led by the same ethnic person, as they believed it would help ensuring that the Pacific concerns are inputted into the system. My study also found that participants' leadership processes were grounded by their perceptions of leadership in general. Similar to Mana, participants viewed leadership as a social process of collective influence within a context, meaning that leadership is depending upon people's actions and interactions to achieve a shared desired outcome.

The comparative analysis of themes emerging from both cases, and theoretical discussions of both cases can be found in the next chapter. 


\section{Chapter 6}

\section{COMPARATIVE ANALYSIS AND THEORETICAL DISCUSSION OF FINDINGS}

This chapter has two purposes. The first purpose is to conduct a comparative analysis of the themes which emerged from the two cases as presented in Chapter Four and Five, and discuss the meaning of these findings in relation to existing leadership theories and studies presented in Chapter One and Two. The second purpose is to present the framework representing the holistic view of participants' leadership processes. Hence the structure of this chapter follows the discussions of two purposes mentioned above.

\section{The comparative analysis and theoretical discussions}

Although there has been some research on organisational leadership processes, very few studies have been undertaken to understand the leadership processes of the current participants from a non-Pacific Public Service context. Some aspects of leadership processes and organisational theory in the public service were shown in this study. This section begins with the comparative analysis and theoretical discussions of organisational themes, then the cultural themes. The organisational themes consist of the participating processes, networking and relationship building, and the possible constraints experienced by participants in their specific organisation. Each of these themes is now examined below.

\section{The participating processes}

Participants' involvement in performing their key roles for both organisations emerges to be one of the primary leadership processes contributing to achievement of desired outcomes in both organisations. The majority of participants from both cases perceived their contribution to organisational outcomes as a collective responsibility, reporting that everyone is participating based on their respective set roles. Participants believed that the communal efforts of staff members to participate collectively, not independently, were 
essential and necessary for achieving the organisations' key roles. In my view, participants' collective responsibility describes their abilities to share the key responsibilities of the organisation, and attempting to empower each others' self-esteem. Most participants from both cases also shared a common attention to engaging in discussing, consulting, providing services to the public, and several leadership roles delegated to them by their superiors. Participants believed that opportunity to experience various duties at different levels enhanced their potential to perform such leadership roles.

However, several differences emerged about the nature of participating processes emphasised by participants of the two organisations. In the Mana case, the nature of the participating process was strongly related to the participants' involvement in the organisation's formal duties. Almost all participants perceived their commitment to facilitate meetings and represent Mana in various official meetings were the key leadership processes they employed to contribute to the organisation's overall outcomes. The usage of meetings, discussions, and consultations indicate the collective participation in decision-making, improving collective cooperation, and means of solving conflicts collectively.

The nature of participating process in the Motu case was largely focused on how participants contributed to the process of decision-making rather than organising and attending meetings. As reported, the organisation consults and engages people from different levels of the organisation in the process of decision-making. A number of participants agreed that the organisation takes into account the communal value of participating in the process of decisionmaking and engaging people from different levels throughout the organisation. According to most participants, such a process values peoples' contribution and enables staff members to have a sense of belonging to the organisation. 
Despite the above differences in participants' perceptions of the participating process across the two cases, it is apparent that participants perceived their involvement in the organisational processes as one of their critical leadership processes. From a theoretical context, Uhl-Bien's (2006) reviews of the leadership literature found that there is a necessity to shift the leadership focus from individual to collective efforts. This was also supported by Yukl (2006) in his research of leadership theories and practises in formal organisations. Yukl describes leadership as a sharing process depending on the participation of the collective.

Important to my study is the notion of leadership as a social process of enhancing the individual to perform collectively, highlighting the importance of collective efforts for producing successful leadership in the organisations. From a public service leadership perspective, Van Wart (2005) argues that allowing members to participate in the organisational processes is one of the leadership styles that should be exhibited from a leader. Van Wart encourages leaders to use various decision mechanisms such as discussing, consulting, and delegating to consider the views of the collective. My participants also reported similar decision mechanisms, believing that allowing such a friendly environment to consider the opinions of the collective is an appropriate way to empower staff members throughout the organisation.

Even though the participating process is supported in the literature, it is important to understand that the existing theories and studies may be focused on the participating efforts of individual leaders or managers, rather than collective efforts. The participating process in my study is quite different because it advocates the notion of starting from the collective and extending to individual efforts. This means that participating is a contribution of all members who are involved in the organisational processes with their set responsibilities and skills regardless of the position held in the organisation. As shown by my study, participants from different levels in the two organisations 
were capable of participating in various consultation processes, using their own techniques of empowerment (like Matelau and Valu), and taking responsibilities when their leaders were absent.

Hence, my study may argue that Van Wart's (2005) decision mechanisms can be utilised by all people at all levels within the organisation, not necessarily by leaders only. Therefore, fitting the participating process in my study within the literature will contribute to increase the understanding that participating is a leadership process of collective efforts and the credit of contributing collectively should be attributed to the general members of the organisation.

\section{The networking and relationship building}

Nearly every participant from both cases agreed on the fact that providing good networking and relationship building had contributed to keeping them focused and well connected. There were two types of networking and relationship building identified in the two cases: formal and informal. With regards to participants' formal leadership processes, the majority of participants from both cases described being involved in networking and relationship building with their internal and external stakeholders in order to raise awareness about the organisations' vision in relation to government priorities. The needs for maintaining good relationships with external supporters were strongly emphasised by most participants in both cases, especially Mana. This reflects on Mana being responsible for developing policies but not implementing them, so the organisation relies heavily on other government agencies for the successful implementation of policies that the organisation formulates. Motu, on the other hand, formulates policies but is also responsible for implementation of these policies throughout New Zealand.

One difference of formal networking and relationship building between the two cases was highlighted by the questions relating to the ways they interacted with other staff members. Almost all participants at Mana reported interacting through internal meetings, organised as a formal mechanism for all staff 
members to meet. Conversely there was no clear description of internal meetings at Motu but it was explained in the organisation's documents that Motu, was involved in various consultations and discussions with its internal stakeholders to support developing the organisations' strategy plans.

On the whole, many participants across the two cases believed that formal networking and relationship building is a continuous strategic process for discussing plans, developing solutions, and implementing policy. In my view, these examples highlight the key leadership processes required by the organisation for participants to perform in order to achieve the organisational desired outcomes. From a theoretical perspective, participants' formal leadership processes of networking and relationship building are traditional styles of managing relationships in the public sector organisations (KoracKakabadse \& Korac-Kakabadse, 1998). In fact, the participants' formal style of networking and relationship building in my study were practised in order to allow open communication and feedback of an issue, and to promote a shared sense of responsibility towards high quality of performance. Likewise, Hosking (1988) refers to this formal networking and relationship building as an organising activity that is developed by staff members of an organisation as a strategy for policy formulation and implementation.

In relation to participants' informal leadership processes of networking and relationship building, findings from both cases showed some similarities in the types of informal networking and relationship building they used. Both cases emphasised high levels of informal networking. The majority of participants from both cases perceived their social interactions with other staff members as an advantage, as they believed it displays high levels of motivation to succeed. The face-to-face communication and understanding people personally were among the popular informal networking and relationship building emphasised by most participants, as they believed it encourages them to work. 
However, there was a difference in the type of informal networking and relationship building available in both organisations. An informal social network exists for Pacific public servants in the Motu case and not at Mana. The majority of participants at Motu believed that having this social network built good relationship among them, helping each others' success, maintaining their Pacific values and interests, and motivating them to succeed in a non-Pacific context.

It is apparent that participants employed their informal strategies of sharing free ideas, information, and advice; personal communication; and informal social networking to develop a friendly relationship-based environment as of assistance to their formal processes. From an organisational context, Murrel (1997) supports this data as an informal collective influential process. Murrel describes,

As leadership is shared and created jointly, so is the responsibility for structuring the organization ... What this means is that people work together to define and develop their relationships not just as questions of influence and leadership, but also as questions of how to keep all of this moving and working together ... how we relate together and influence each other (1997, p. 40).

It appears that networking and relationship building is established through sharing and joining together where people are working together in a way to generate influence at a collective level. Participants also agreed that building a good relationship is an outcome of networking and interacting collectively. In my view, participants' actions to build their own informal networking and socialising system outside the organisation's formal networking showed their active move to justify their values and interests with interested members. These advantages of informal networking and relationship building are compatible with Hosking's (1988) networking skills, believing that it is useful to develop interested members' knowledge and to demonstrate their beliefs in action. 
This finding also sustains Balkundi and Kilduff's (2005) idea that networking is structured cognitively in the minds of individuals. In fact, participants value what appears to be important in their mind and behaviours that are embedded in networking and interpersonal relationships.

The emerging theme of networking and relationship building in my study shows that Pacific participants' leadership process is not restricted to a single or even a set group of executives, but includes the associated actions of the collective. While the leadership process of networking and relationship building is supported in the literature, very little information is known about the informal networking and relationship building of a minority social network in a foreign public service context. This finding makes the leadership process of networking and relationship in my study different from that in the literature. Although participants' leadership processes of participating, networking and relationship building have been considered here as organisational leadership processes, to a great extent, they are culturally driven as well. I will consider participants' cultural leadership processes further in this chapter.

The possible constraints on participants' organisational leadership processes Almost all participants from both cases perceived their current leadership processes to be constrained by various organisational factors. These factors could be classified in two types, the external and internal factors. The common external factors included the influential power of the Minister and executive members. This was a major concern for the participants because they believed the power of the government as conferred to the responsible Minister, who then directs the entire organisation to work systematically, paying less attention to peoples' emotions. This data is supported by Loverd (1997), saying that leadership in the public sector is power-oriented. This means that whoever has the leading position in the organisation has the power to make directions. As reported by participants, the government is also subject to change depending on elections and this can influence how the organisation operates. 
This study found some similarities of internal organisational factors between the two cases. Job complexities such as workload, its urgency, organisational structure, and the uncertainties relating to decision making by executives regarding restructuring were the internal factors which most frequently cited to have a negative effect on participants' leadership processes across the two cases. According to many participants, the effects of working in a systematic organisational structure, including being controlled by regulations and set roles tended to create few chances for innovation and assessing their work critically. Participants also saw restructuring as a demotivator.

Findings regarding the influence of job complexities on my participants' duties is supported in research by Mumford and colleagues (2000). In their study with officers in the United States Army, Mumford and colleagues found that the challenge of assignment loads and variety of tasks influenced officers' learning from experience and development of leadership skills. While the majority of public service organisations are still dominated by hierarchical structure (Parker \& Bradley, 2000), more studies are needed on effects of organisational structure on my participants' leadership processes.

There was a difference in how the issues of restructuring influenced participants of the two organisations. The outcome of internal restructure occurring at Mana caused huge uncertainties for almost all participants in that case. They were concerned about the loss of experienced people, believing that those who left the organisation still had valuable skills for the organisation. It was different in the Motu case although it was undergoing change as well. Participants at Motu did not have great influence on change but it appeared to be a concern for most participants, in particular the negative effects of this change on colleagues' motivation, especially those who had worked in the organisation for years. 
To address the uncertainties in the process of internal restructure, most participants from both cases believed that transparency should be emphasised. Before the full implementation of internal restructure, participants believed that members of the organisations should be provided with clear information about the change, why it is needed, and how it is going to be implemented. Participants argued that everyone in the organisations should be informed accordingly regardless of the positions they hold and the types of work they do. Once the restructure is implemented, participants felt that reconciliation should be conducted with the remaining staff.

Within the existing research, the problems with the uncertainties of change have been addressed by Parry's (1999) theory of enhancing adaptability. Parry describes enhancing adaptability as a social process such as face-to face communication which leaders employ to improve the adaptability of themselves and their followers in a turbulent change situation. The challenges experienced by participants in this study are comparable with the findings of research conducted by Kan and Parry (2004) in New Zealand. Their study found that the leadership processes operating within the nursing environment of a hospital which was undergoing change was driven by societal factors within and outside nursing. As they reported, such factors hold back the potential of nurse leaders to achieve greater influence within that context. My study points to the need to give attention to how the organisational constraining factors affect leadership processes of ethnic group members in a foreign public service context, as I believe that the above constraining factors may suppress participants' abilities to employ successful leadership processes.

It is not within the scope of my study to examine the complexities of organisational factors in relation to participants' leadership processes. However, I hope that the findings of my study inform decision and policy makers in the public service about different factors affecting the leadership capability of Pacific public servants as a minority in New Zealand. The next 
section presents the cultural themes, comprising four key themes: the participants' learning experiences about leadership, practising of $v a$, conceptualising leadership, and working in New Zealand. These themes are discussed below.

\section{Learning about leadership from cultural contexts}

The results show two similarities between the two cases in relation to learning. The first similarity is that almost all participants from both cases credited the formation of their leadership learning as an outcome of cultural contexts. Participants described family, church, and social related informal learning experiences as providing their main experience of learning about leadership, as they believed it helped them in the absence of formal leadership training and when they had to take over a leader's responsibilities.

The second similarity is the leadership support available for participants in the New Zealand Public Service. The results of interviews indicate that, in general, formal leadership training in the public service is available for organisation leaders only. Eleven of the sixteen participants had no formal leadership support in their organisation. In many cases participants reported preferring to seek the leadership support of their Pacific networks. Conversely, there was a difference in the leadership support available between the two cases. It was evident that there was a leadership workshop available at Motu, but participation was limited because the entry to this programme is based on certain qualifications and requirements. In contrast, at Mana there is no similar opportunity available specifically for Pacific public servants. This supports the State Service Commission's (SSC, 2004) report that there is insufficient leadership development available for Pacific public servants in New Zealand.

Participants perceived their informal learning experience about leadership as of useful to their performance in New Zealand. It is apparent that participants did use their informal leadership skills obtained from their cultural contexts to perform their duties and provide other staff members with greater information 
about the values of their cultural leadership. Participants concurred on the significant contribution of social interactions in guiding them in the organisations. This finding reveals the cultural make up of the Pacific public servants. Almost all participants from both cases held a high opinion of the importance of informal learning in their performance at Mana and Motu, believing that it provides them with the confidence to deal with the complexities of their job.

From a theoretical context, learning from experience is a useful way of obtaining leadership knowledge and skills, as it is about the interactions between the organisation's members and their environment (Davies \& Easterby-Smith, 1984). Though Davies and Easterby-Smith looked at the usefulness of informal learning from managers' perspectives, central to this study is that they found that manager skills and knowledge for effective leadership in organisations came from their experience, rather than formal training programmes. Participants believed that learning by action is more appropriate than just awareness and perceptions. The majority of participants believed that opportunities allowing them to partake in the leadership process enhanced their learning capacity and confidence. Sanga and Walker (2005) describe learning by action as an example of an influential process. They said, "people are more powerfully influenced by good actions than by expressions of good intentions" (Sanga \& Walker, 2005, p. 28).

With the questions relating to the content of leadership training, nearly all participants from both organisations wanted to include their cultural components of leadership, agreeing it will raise national awareness about the value of Pacific cultural leadership. The participants also wished to gain more knowledge and skill from formal leadership training. In my view, this may reflect on the fact that leadership in the public service builds on certain skills in order for them to succeed. 
Although the informal learning from experiences in formal organisations is supported in the literature, very little research can be found on the usefulness of learning experiences from cultural contexts in relation to leadership processes in formal organisations. Hence incorporating this finding into the leadership literature in the public service raises awareness that participants' informal learning from cultural contexts is significantly important in formal organisations.

\section{Practicing the Pacific value of $v a$}

The leadership concept of $v a$ was used to help group together some shared ideas of the participants regarding the importance placed on respecting others. Although the term $v a$ was only used by participants at Motu, the practice of $v a$ in terms of considering the social value of people shaped how most participants of both cases employed their leadership processes. Participants believed that practising $v a$ in the workplace gave them a sense of self-importance, inspiration, and kept the momentum in their performance. The majority of participants from both cases believed that the norms of their cultural background were still relevant in a non-Pacific context like New Zealand. This is related especially to the way they interacted with other members in the organisations.

$V a$ is supported by the Confucius principal, claiming that respecting others is an ethical set of beliefs that are practised by people in their daily lives since birth (Hofstede \& Bond, 1988). Practising $v a$ is a basic human kindness which includes "treating others as one would like to be treated" (Hofstede \& Bond, 1988, p. 8). There is a parallel of practising $v a$ with love, as an opportunity to improve leadership in an organisation (Rodney, 1988). Although Rodney did not refer to the notion of $v a$, he sees love not from the traditional mind-set of romantic love but thinking deeply about the social value and leadership potential of people in the organisation. In my view, participants' leadership process of $v a$ advocates understanding, caring, helping, listening, and believing the potential of people who are capable. In a recent study, va was viewed from an aesthetic leadership perspective or the consideration of feeling and emotion 
as important aspects of organisational behaviour (Hansen et al., 2007). Hansen and colleagues believe that emotion and feeling are sources of knowledge and it generates meanings that guide peoples' behaviours. In fact, my participants had similar thoughts, believing that most people are relying on the benevolence of $v a$ to guide their actions and thoughts.

In my view, $v a$ is instilled with the cultural significance of participants' respect for the dignity of others. The participants' practising of $v a$ toward other people through interacting considerately with them, and understanding their leadership structure, introduced this theme as being at the heart of almost all participants' leadership processes. Culture may be just a vehicle for participants' leadership processes. The inclusion of participants' learning experiences from cultural contexts and practising $v a$ are important indicators of the ethnic make-up of the participants, and highlights the ethnically distinctive values of Pacific people in the New Zealand workplace. Although practising va is supported in the literature, the understanding of $v a$ as 'allowing a space to develop the potential of each person' adds new perspective of leadership process to the leadership literature.

\section{Participants' perceptions of leadership in general}

No major differences were identified among participants' perceptions of leadership in general. Participants' perception of leadership plays an important role in how they undertake their leadership processes. Generally, participants from both cases conceptualised leadership as a social process of collective influence within a context. This definition has four aspects of leadership concept. The first aspect views leadership as a social process, which is an action or inter-action among staff members of the organisation. The second aspect understands leadership as collective efforts. The third refers leadership to the process of influencing or persuading others to move, and finally, leadership occurs within a context. Participants also saw the need to promote two-way flows of relationship in the public service leadership, meaning that leaders influence and are influenced by members of the organisation. 
Almost all participants from Mana and Motu believed that leadership exists as a collective involvement where all people are involved but in different contexts. Participants concurred that leadership is necessary for guiding people to move towards achieving the organisational desired outcomes, as they believed it produces productive organisations.

This understanding of leadership is similar to several theories and findings in the literature. Those who advocate leadership as a process in organisational settings include Bratton, Grint, and Nelson (2005), and Yukl (2006). Bratton and his colleagues describe leadership as a social process that is constructed through the interaction of leaders and followers in a context. Similarly, Yukl sees leadership as a process of social influence. From a public service context, leadership is also defined as a process of social influence (Parry, 1998). Whilst these theorists view leadership as a social process, the involvement of the collective in the leadership process is still unclear. This gap highlights the distinctiveness of conceptualising leadership in the current study, as participants believed that leadership in their organisation is a collective responsibility.

\section{Working in a non-Pacific context}

Participants from both cases generally considered that being of Pacific descent had a positive effect on their work and leadership experiences in New Zealand, as it gave them confidence in their performance at Mana and Motu. This data is supported by Elkin and colleagues' (2004) understanding of organisational behaviour in New Zealand. They found that Pacific workers carried a lot of

their cultural values into the workplaces, as I believe we all experience but it becomes noticeable when we are in different cultural contexts. While coming from the Pacific nations benefited the development of almost all participants' leadership experiences, very few of them reported that their leadership success in New Zealand was not related to ethnicity. Instead, their professional success was developed from the quality of performance and their individual adaptability to the environment. 
Whilst participants' cultural background contributed to their leadership capacity, they simultaneously felt somewhat limited by cultural difference they experienced in a non-Pacific environment. The cultural difference of individualism versus collectivism was a concern for most participants because it might cause some conflict of views and expectations between the Pacific and Palangi people. This data has been found by Hofstede and Bond (1988) in their global cross-cultural research. Hofstede and Bond found that people in most foreign countries tended to accept that reality of leadership is individually driven, meaning that everyone must look after herself or himself individually. However, on the collective side, it considers that everyone in the family or organisation has responsibility to integrate strongly and build consistent relationship among members from birth or from starting in the organisation.

Different to Mana, the majority of participants at Motu are concerned with how effective the New Zealand Public Service is in their responsibility to improve Pacific peoples' outcomes in New Zealand. They reported experiencing a lot of tokenism in key role of public service organisations for improving Pacific peoples' outcomes in New Zealand. They felt that most Pacific frameworks were not fully implemented by public service organisations including Motu, believing that the implementation of Pacific strategies was of less priority for most mainstream organisations. This issue was not raised at the Mana case.

While most participants wanted to work in their own comfort zone, it was evident that being a Pacific leader in a non-Pacific organisation is not a matter of being a Pacific descent. I found it was a matter of complying with the public service requirements and controlling the organisation according to what the dominant culture believes is appropriate. This supports what Loverd (1997) refers to as a power-oriented style of leadership. Loverd insists that although there are contradictions at all levels when the policy is passed to the organisation level, top management people are still expected to support the views of their leaders who are generally political appointees. 
The above also supports the "power distance" between the dominant culture and the minority culture (Hofstede \& Bond, 1988, p. 10). This means that the less powerful members of the organisations or the minority people of a society concur on the fact that power is distributed unequally and tend to follow what the people of dominant culture are attempting to promote. In general, the findings on leadership challenges experienced by my participants in New Zealand may sum up the controversy about the use of power by the dominant culture to control over information to bias minority perceptions about a situation (Yukl, 2005).

This study, therefore, points to the necessity to fully utilise the leadership potential represented by diverse members of the organisation (Yukl, 2006). I agree with Yukl on the fact that it is crucial to remove constraints that prevent potential people from qualifying to important positions in the organisation. It is evident in my study that participants bring with them valuable leadership experiences but there is a mismatch with the leadership expectations in New Zealand. This mismatch may suppress the leadership potential of Pacific public servants.

\section{The conceptual framework of participants' leadership processes}

The above comparative analysis indicates that the leadership processes experienced by participants were closely interrelated across the two cases. In this section, a framework representing the holistic view of participants' leadership processes is illustrated in Figure 1 below.

The framework conceptualises my understanding of leadership processes experienced by participants across the two cases. The combination of organisational and cultural key leadership processes formed the basis of Pacific public servants' leadership processes, depicted in the green circles of Figure 1. As it appears, participants' leadership processes are centred on four key areas: their participating, networking and relationship building, learning about leadership from cultural contexts, and practising the Pacific value of $v a$ at the 
workplaces. All these processes highlight the participants' understanding of leadership as a social process of collective influence within the public service context. Whether they are under the organisational or cultural leadership processes, it should be noted that the dotted lines show their interrelation.

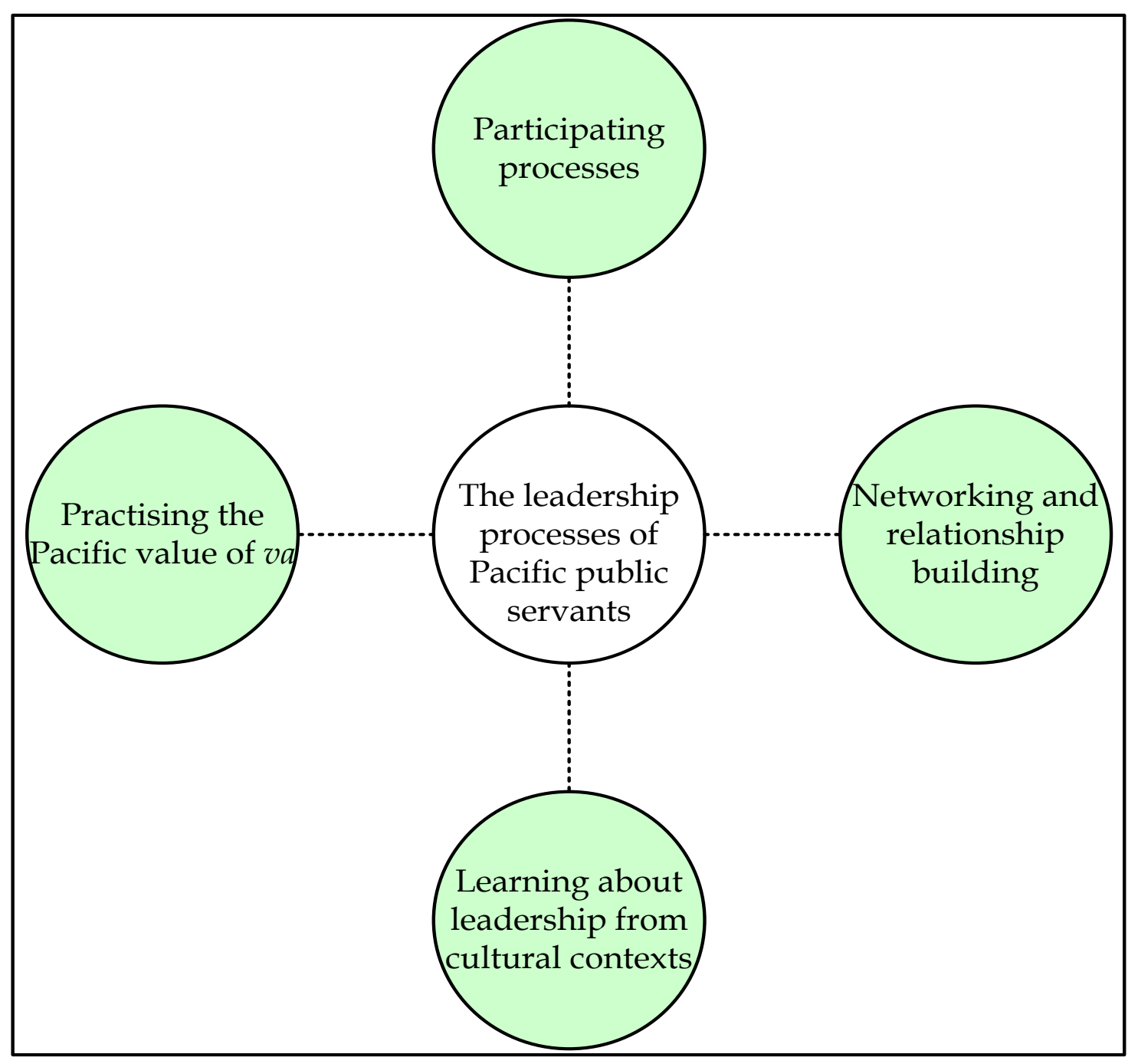

Figure 1: The conceptual framework of Pacific participants' leadership processes in the New Zealand Public Service organisations

In detail, the participating process indicates that in order for participants to succeed in the two organisations, they are required to be involved in various activities to provide services and to fulfil their set responsibilities for the organisations as a whole. Networking and relationship building were commonly practised by participants to disseminate the core functions of the organisation throughout the organisations and to strengthen their relationships with internal and external stakeholders. Further to that, participants applied, in 
their everyday tasks, the leadership skills and knowledge they gained informally from cultural contexts. Participants also practised the Pacific value of $v a$ frequently in their interactions with other staff members.

These leadership processes of Pacific participants demonstrate Van Wart's (2005) Leadership Actions Theory described in Chapter Two as the theoretical foundation of this research. These included task-oriented, organisationaloriented, and people-oriented leadership processes. The participating leadership process of Pacific participants is task-oriented, emphasising the activities that staff members were involved in to achieve the organisational outcomes. Networking and relationship building have some organisationaloriented aspects, highlighting activities such as meetings that are established by an organisation to build its relationship with key stakeholders. What was different in the findings of this theme is the emergence of participants' informal Pacific networking as a way to value their cultural interests and encourages them in a non-Pacific context. The leadership processes of participants' learning about leadership from cultural contexts and practising the Pacific value of $v a$ are in line with Van Wart's (2005) people-oriented approach. This approach gives emphasis to people's capabilities.

Generally, the Pacific participants' leadership processes of participating, networking and relationship building, learning about leadership from cultural contexts, and practising $v a$ are task-oriented, organisational-oriented, and people-oriented in nature. However, the aspect of participants' cultural leadership processes makes the findings of my study slightly different from the existing research of leadership processes in the public service. It emphasises the fact that leadership processes of minorities in a foreign public service context may be driven from the organisational key roles but, more importantly, from their ethnic background. 
The findings bring to my attention the fact that the leadership processes of Pacific participants are a challenge to formal structural processes in the public service organisations. In my view, this means that leadership as a social process brings light to the public service context, arguing that structural leadership processes may not necessarily be the only way to guide people within the organisation.

The present study also found various organisational and cultural factors that somehow contribute or constraint participants' leadership processes. These factors are presented in Table 4.

\begin{tabular}{|c|c|c|}
\hline & Contributing Factors & Constraining Factors \\
\hline Organisational & $\begin{array}{l}\text { - Government priorities, and } \\
\text { - Key roles of the organisation. }\end{array}$ & $\begin{array}{l}\text { - External factors such as the } \\
\text { power of the Minister and } \\
\text { elected Government, } \\
\text { - Internal factors such as the } \\
\text { organisational structure, jobs } \\
\text { complexities, and the } \\
\text { uncertainties of decision- } \\
\text { making about restructuring } \\
\text { positions, and } \\
\text { - Lack of leadership support } \\
\text { available for participants at all } \\
\text { levels in the New Zealand } \\
\text { Public Service. }\end{array}$ \\
\hline Cultural & $\begin{array}{l}\text { - Participants' general } \\
\text { leadership experience, } \\
\text { - Participants' perception of } \\
\text { leadership in general, and } \\
\text { - Cultural values of } \\
\text { participants' Pacific } \\
\text { leadership. }\end{array}$ & $\begin{array}{l}\text { - Cultural differences between } \\
\text { the Palangi and Pacific people } \\
\text { style of leadership, } \\
\text { - Foreign environment, and } \\
\text { - Power of dominant culture. }\end{array}$ \\
\hline
\end{tabular}

Table 4: The organisational and cultural contributing and constraining factors of Pacific participants' leadership processes.

The contributing factors support participants' leadership processes in the two organisations. The cultural factors that were contributing to the successful implementation of participants' leadership processes included their leadership experiences, leadership perceptions, and cultural values of Pacific leadership. 
The government priorities and key roles of the organisation also contributed to shaping participants' organisational leadership processes. The constraining factors inhibit participants' potential to promote their leadership processes at Mana and Motu. The cultural constraining factors included cultural differences, foreign environment, and the power of dominant culture. From the organisational side, the power of the government and Minister were the major external factors. The internal factors constraining participants' organisational leadership processes included the organisational structure, job complexity, the uncertainties of decision making relating with restructuring, and lack of leadership support available for them in the New Zealand Public Service.

In general, Table 4 above shows areas where the strengths of participants' leadership processes are embedded, and areas requiring development to enable the participants to fully utilise their leadership potential in a non-Pacific context. The constraining factors highlight the areas in which Pacific public servants may need leadership support giving notice to New Zealand Public Service organisations to be aware of the problems they experience in the workplaces.

\section{Summary}

Overall, the results of the findings from my study revealed some shared themes of leadership processes experienced by participants in the two organisations. They were the leadership processes of participating, networking and relationship building, learning about leadership from cultural contexts, and practising the Pacific value of $v a$. These shared themes came from the organisational key roles and participants' cultural values. They were also shown to be socially constructed among the participants and their interactions with other staff members within their specific organisations. However, the extent in which participants employed their leadership processes effectively in a non-Pacific context was also shaped by other factors. These included the contributing and constraining factors of the organisations and cultural background. 
The next chapter concludes this thesis with the key findings of my study, in relation to the research question and objectives, my reflections on the current study, proposals for future actions and researches, and the contribution of my study to the leadership literature. 


\section{Chapter 7}

\section{CONCLUSION AND RECOMMENDATIONS}

This chapter concludes this dissertation with several purposes. First, I review the research question and what I have achieved in terms of the research objectives. Secondly, I reflect on what I have learnt from the current study. Thirdly, I provide recommendations to the public service on issues relating with the Pacific public servants' leadership processes, and for future research. Finally, I discuss the contribution of my research to leadership process generally.

\section{Review of the research question}

As mentioned in previous chapters, my study explored this research question: What are the leadership processes currently employed by Pacific public servants in the New Zealand Public Service? This question was examined using in-depth interviews with the participants, and supported by a document analysis of organisational documents.

The most essential underlying theme in the current study is the participants' emphasis on the perspectives of leadership processes that were crucial to their performance in the two organisations. The present study revealed four key leadership processes experienced by Pacific public servants in the New Zealand Public Service. The first leadership process is participating. The participants' report on their key leadership activities in the two organisations indicated that they were involved extensively in the organisational processes to fulfil the key roles of the organisations. Participants engaged in various forms of decisionmaking processes such as delegation, consultations, discussions, and meetings to fulfil various needs for the organisation. 
The second leadership process was networking and relationship building. The strength of networking and relationship building of the leadership process rests on strengthening the organisation relationship with key stakeholders. Informal networking and relationship building appeared to be the fundamental leadership process of social influence essential for almost all participants. Participants' responses showed that they were doing well in employing informal networking and relationship building process to interact with others, but somehow limited by structure of the organisation, their formal positions, and conflict views between them and their non-Pacific colleagues.

Thirdly, participants indicated that they made successful use of the leadership experiences learnt informally from their cultural contexts to have the confidence for fulfilling duties. It was evident in my study that formal leadership skills were useful but somehow limited, as participants believed they are not enough to guide people in the organisation. Therefore, participants' informal learning about leadership from cultural contexts was considered the most useful experience for their work.

Nevertheless, informal leadership learning had certain limitations for the professional needs in the public service organisations. To improve this, almost all participants agreed that providing more leadership activities for them at all levels may well equip their informal learning experiences to meet the professional needs in the mainstream organisations. Participants displayed a high demand of leadership support from their Pacific networks, preferring the help of Pacific public servants in the executive level. Participants' preferences to expose their cultural leadership concepts in the content of a leadership workshop highlighted their ethnic make-up. This finding gives attention to the need for public service organisations in New Zealand to provide appropriate leadership support for the increasing numbers of Pacific public servants who have valuable cultural leadership skills, different from those of the Westernbased leadership learning. 
The fourth leadership process was practising va. It was evident from the present study that participants' high level of understandings the nature of their cultural leadership style still very much influences their leadership processes at work. Participants believed that practicing va was among the most significant leadership process for them. In my view, practising va signifies participants' cultural belonging, cultural means of inspiring others, and its strength to give participants confidence to interact effectively with co-workers. The leadership process of practising $v a$ also indicates participants' additional care for people around them. However, the limitation of this leadership process is that participants often faced difficulties with how their culturally based style of leadership is received by Palangi people.

The above findings show that I have achieved the research objectives mentioned in Chapter One. The first objective achieved an understanding that Pacific participants perceived the participating process, networking and relationship building, learning about leadership from cultural contexts, and practising the Pacific value of $v a$ as important leadership processes to their work at Mana and Motu.

My findings also achieved the second research objective with an understanding that the participants' cultural background had a positive effect on their experience of leadership processes in New Zealand. As documented in this thesis, the participants' cultural understanding of leadership was the fundamental key in their leadership processes in the two organisations. Despite the lack of recognition of Pacific leadership skills in the two organisations being studied, the majority of participants still insisted on employing their cultural values and acknowledged it as part of their contribution to their jobs. It is also important to understand that participants bring into non-Pacific Public Service organisations their own leadership strengths and weaknesses that are worth considering. Although participants' leadership processes were not driven solely 
from their cultural values, my study provides deeper understanding about the usefulness of Pacific leadership to the New Zealand Public Service.

The findings that emerged from the present study also achieved my third research objective. As the results show, participants perceived that the government priorities for the case organisations' key roles are contributing factors to their leadership processes. My study also found that the organisational constraining factors in Table 4 above may be out of the participants' control. However, the results that emerged from my study indicate a relative lack of attention by the literature on public service leadership to how foreign organisational contexts affect specific leadership potential of a minority. To ensure that Pacific public servants have access to appropriate leadership opportunities in New Zealand Public Service organisations, it must be recognised that they are not only valuable resources in New Zealand but also that they do face difficulties in utilising their leadership processes in a nonPacific context.

\section{My reflections on the current study}

I have four major reflections about the findings of the current research. The first reflection was that the findings of the current study are limited to the organisations and region in which this study occurred. These organisations were government departments in the Wellington region. It should be understood that participants' data and findings from the organisational documents may not apply to other organisations and regions in New Zealand.

Secondly, although participants were known in the current study as 'Pacific' public servants, it should be noted that half of the participants were identified as being Samoans, and their experiences of leadership processes may dominate the overall findings of this study. With the exceptions of the Tokelauans, the other participants were Tongans, Niueans, a Cook Islander, and a Fijian. Further studies with more participants from other Pacific ethnic groups are needed. Thirdly, if I were given a chance to re-conduct the current study, I 
would add a focus group of Pacific public servants to my data collection tools. I believe this might bring different insight to the research findings.

Finally, the confidentiality aspect of the discussions in my study led to limited useful information such as vision of the organisations that would have contributed to a greater understanding of participants' leadership processes. In addition, the confidentiality of information about the total number of Pacific staff at each New Zealand Public Service organisation did not give me enough information of how the Pacific public servants are distributed. This is a lesson learnt for consideration in future studies.

The above reflections do not indicate that the research findings in the current study are not trustworthy. In fact, it has challenged me to provide possible recommendations for New Zealand Public Service organisations on leadership supports and development appropriate for Pacific public servants, and directions for future researchers.

\section{Recommendations of support for Pacific public servants}

Since one of the New Zealand Public Service's strategic goals is to provide equal opportunities for achieving public servants' leadership capacities at all levels (SSC, 2004), examining Pacific public servants' leadership processes is important. In fact, Parry and Proctor (2000) suggest that understanding of leadership social processes and the way they are operated within New Zealand organisations will be useful for understanding the uncertainties and turbulence within the organisational culture. As a result of the findings from the current study, a number of issues arose, suggesting the following recommendations for public service organisations to provide leadership support appropriate for Pacific people in a non-Pacific context.

The first is encouraging social support for enhancing individual leadership capability but integrated in a collective understanding of leadership, and to provide continuous supports and opportunities for leadership development. To 
address the issue of individual leadership constraints, it may be appropriate to give Pacific participants the opportunities to demonstrate leadership actions in their everyday duties, and allow them to listen and access to the leadership experiences of live people within the public service. Learning from experience is a worthwhile skill and allowing public service leaders to share their leadership experiences with Pacific public servants will surely add value to their performance. In the long-run, this social assistance would be better equipped with extensive professional leadership supports for Pacific public servants at all levels throughout their professional careers in the public service.

Secondly, my study found that numerous valuable leadership skills and knowledge were experienced by participants from a range of cultural contexts. Unfortunately, they are seldom considered as performance qualities in the professional appraisal system of the case organisations. This lack of recognition of Pacific participants' cultural leadership skills can have negative effects on their motivation, causing them to feel down about their valuable contributions to the organisation. To address these problems, there is need for a tangible and long-term approach to consider cultural leadership skills in the public service human professional development. Although there was an attempt by the State Services Commission (2004) report to address the cultural barriers experienced by Pacific public servants in a number of strategies, the question of its effectiveness still remains unchanged. Given that lack of valuing cultural competencies still exists after about four years since the State Services Commission's report, there is doubt about the effectiveness of the New Zealand Public Service's efforts to implement those strategies.

Thirdly, the current study indicated that the Pacific participants' demand for the continuous involvement of their Pacific networks. To address this issue, it may be appropriate to have a formal and long-term strategy that aims at assisting Pacific public servants by providing further leadership assistance from their social networks. This would enhance their motivation at work, raising self- 
esteem, and recognise their value as being public servants in New Zealand organisations. The public service organisations they work for would also benefit in terms of their achievements and progress.

Finally, the findings revealed that the participants had limited opportunities to create innovation especially in leadership experience, as they are highly controlled by the power of hierarchical structure and various set guidelines. These are long standing issues in almost all public service organisations but worker resistance to such an organisational culture should be taken into account knowing its negative effect on their attitudes to work. In the long-term, the organisational culture should be structured in a friendly environment that would promote the Pacific public servants' leadership potential to exist within that structure. To ensure effective implementation of the strategies, ongoing follow-up should be emphasised at all times and in a culturally appropriate way that would benefit the Pacific public servants and their performance in the public service.

Likewise, the Pacific public servants in the current study were discouraged by the lack of Palangi people's understanding of their collective Pacific cultural way of experiencing leadership. These gave them little room to disseminate their collective leadership process of working and making decisions as a group and establishing their capacities to succeed. These issues may be a never-ending battle but the public service organisations cannot afford to neglect such a concern considering the increasing number of Pacific public servants being recruited to the public service. One way to address the issue of individualism and lack of understanding of the Pacific ways may be to directly teach nonPacific people about the important of collective influence for Pacific peoples' leadership, and the leadership diversity that exists among Pacific ethnicities. Moreover, one of the most disturbing processes that worried almost all participants at the time of the study in the two organisations is the uncertainties of decision-making relating to organisational restructuring. To solve these 
uncertainties, participants believed that leaders and those who make decisions in the organisation must ensure there is clarity for the entire organisation regarding any restructuring. This must occur before the transition process, and reconciliation must be conducted after change has been implemented to rectify any possible problems that occurred. The other possible solution may be to allow all members' to provide their views about the restructuring process, regardless of their positions. These would give implications for leaders in terms of decision-making process.

\section{Recommendations for future research}

Since Pacific people are well represented in New Zealand Public Service organisations and leadership is one area of focus for all public servants' achievements and progress, the case organisations must be mindful about the specific leadership supports relevant for Pacific public servants. The results from the current study strongly indicate the necessity to give more attention to the role of participants' leadership processes as a major factor contributing to their leadership behaviour in the organisational contexts. As a result, the current study highlighted some important issues for future studies.

The first is related to the need to provide more comprehensive research of Pacific public servants' leadership processes. Since the goal of the current study is to gain a better understanding of Pacific public servants' leadership processes, more detailed studies of Pacific public servants from all Pacific ethnic groups are necessary. Likewise, further study of Pacific public servants from other public service organisations and regions would be needed for deeper understanding of their leadership processes. This is because it was evident that their leadership challenge in the New Zealand Public Service may differ according to the nature of leadership approach emphasised in the organisations in which they work. 
Secondly, a future research should be focusing on examining a particular leadership process such as practising va and learning about leadership from cultural contexts and identify their specific relationships with Pacific public servants' performance. Focusing on a select group of leadership processes in depth could elicit more meaningful results.

Thirdly, there is a need for a study on how to integrate the Pacific public servants' cultural competencies into the human resources professional leadership development in non-Pacific mainstream organisations. Such a study may attempt to answer questions such as what are the Pacific cultural competencies relevant for professional leadership development, in what ways they are going to be implemented, who are the appropriate people to guide the implementation stage, and other relevant questions that will provide long-term leadership support for Pacific public servants in New Zealand.

Finally, research which studies the differences in leadership process between Pacific public servants and Pacific people in the New Zealand Private Sector is needed to identify the central leadership processes used by them to cope with their performance in a non-Pacific context.

For the benefit of the future researchers who wish to conduct studies involving Pacific participants, my experience from this study confirms the importance of paying special attention to participants' cultural background. Future researchers must be mindful of the Pacific ways of doing things and their strong cultural belief as important for designing of their research. In doing so, it balances the researchers' respect for the Pacific culture as well as the mainstream culture of any foreign context. Designing the research strategies to match with the cultural aspects of Pacific people is important; whether it is a qualitative or quantitative study, it must be conducted in appropriate cultural way. 


\section{The contribution of my research to the leadership processes generally} The key research findings contribute to the field of leadership study in two areas. The first is related to the cultural contribution of the research findings to the literature of leadership processes in the public service. The current study provides in-depth understanding that Pacific public servants' perceptions of leadership and leadership processes were culturally focused. While participants' leadership processes of participating and networking and relationship building were considered under the organisational leadership processes, it was evident in my study that they are also driven by participants' cultural views. As highlighted in the findings, participants' understanding of leadership and their perceptions of leadership processes were centred on collective actions, a Pacific means of doing leadership. This indicates the distinctiveness of collectivism in my study, not only for Pacific public servants but central to Pacific leadership. Participants' leadership processes of learning about leadership from cultural contexts and practising $v a$ bring special contribution to the leadership literature in the public service.

The second contribution of the current study relates to the practical issues regarding the Pacific public servants' leadership processes. Participants' leadership needs challenge the public service to provide effective leadership support that is sensitive to their cultural values. In addition, my research findings also challenge decision-makers in the New Zealand Public Service to put into effect the implementation of existing leadership strategies available for Pacific public servants.

On the whole, this study fills an important gap in the leadership process of this local minority in the New Zealand Public Service. The topic is very useful and it needs further research because very little investigation has been done on the leadership processes of Pacific public servants. The findings can be generalised in the field of public service leadership. My study provides insight to current and for future Pacific public servants in New Zealand. Although the findings 
from my study might be biased to the views of the Pacific public servants who participated, it is apparent that their leadership issues in the public service are common and widespread. Generally, this finding reflects what the literature found about the organisational and cultural difficulties many professional workers faced. In light of the findings, public service organisations in New Zealand should be aware of the problems that affect Pacific public servants' leadership experiences while struggling to perform their best in all responsibilities required of them in their respective organisations. 


\section{REFERENCES}

Ah Chong, L. M., \& Thomas, D. C. (1997). Leadership Perceptions in CrossCultural Context: Pakeha and Pacific Islanders in New Zealand. Leadership Quarterly, 8(3 ), 275-293.

Alimo-Metcalfe, B., \& Alban-Metcalfe, J. (2004). Leadership in Public Sector Organizations. In J. Storey (Ed.), Leadership in Organizations: Current Issues and Key Trends (pp. 173-202). New York: Routledge.

Anae, M., Coxon, E., Mara, D., Wendt-Samu, T., \& Finau, C. (2001). Pasifika Education Research Guidelines. Auckland, New Zealand: The University of Auckland.

Balkundi, P., \& Kilduff, M. (2005). The Ties that Lead: A Social Network Approach to Leadership. The Leadership Quarterly, 16, 941-961.

Ballard, L., \& Kleiner, B. H. (1988). Understanding and Managing Foreign-Born and Minority Employees. Leadership and Organization Development Journal, 9(4), 22-24.

Bass, B. M., \& Riggio, R. E. (2006). Transformational Leadership (2 ed.). New Jersey: Lawrence Erlbaum Associates Publishers.

Brain, K., \& Lewis, D. (2004). Exploring Leadership Preferences in Multicultural Workgroups: An Australian Case Study. Leadership and Organization Development Journal, 25(3), 263-278.

Bratton, J., Grint, K., \& Nelson, D. L. (2005). Organizational Leadership. Ohio, United States: Thomson.

Burns, J. M. (1978). Leadership. New York: Harper and Row Publishers.

Cavana, R. Y., Delahaye, B. L., \& Sekaran, U. (2000). Applied Business Research: Qualitative and Quantitative Methods. Queensland, Australia: John Wiley \& Sons.

Churney, R. (1998). Political Culture and Political Process: Fijian, American Samoa, and Palau. In D. Shuster, P. Larmour \& K. V. Strokirch (Eds.), Leadership in the Pacific Islands: Tradition and the Future (pp. 115-125). Canberra: The Australian National University. 
Constas, M. A. (1992). Qualitative Analysis as a Public Event: The Documentation of Category Development Procedures. American Educational Research Journal, 29(2), 253-266.

Cox, T. J. (1991). The Multicultural Organization. Academy of Management Executive, 5(2), 34-47.

Creswell, J. W. (2007). Qualitative Enquiry and Research Design: Choosing Among Five Approaches (2 ed.). Thousand Oaks, CA: Sage

Davies, J., \& Easterby-Smith, M. (1984). Learning and Developing from Managerial Work Experiences. Journal of Management Studies, 21(2), 169183.

Denscombe, M. (2007). The Good Research Guide for Small-Scale Social Research Projects (3 ed.). New York: Open University Press.

Denzin, N. K., \& Lincoln, Y. S. (2005a). Introduction The Discipline and Practice of Qualitative Research. In N. K. Denzin \& Y. S. Lincoln (Eds.), The Sage Handbook of Qualitative Research (3 ed., pp. 1-28). Thousand Oaks, CA: Sage.

Denzin, N. K., \& Lincoln, Y. S. (2005b). Paradigms and Perspectives in Contention. In N. K. Denzin \& Y. S. Lincoln (Eds.), The Sage Handbook of Qualitative Research (3 ed., pp. 183-190). Thousand Oaks, CA: Sage.

Denzin, N. K., \& Lincoln, Y. S. (2005c). Strategies of Inquiry. In N. K. Denzin \& Y. S. Lincoln (Eds.), The Sage Handbook of Qualitative Research (3 ed., pp. 375-386). Thousand Oaks, CA: Sage.

Elkin, G., Jackson, B., \& Inkson, K. (2004). Organisational Behaviour in New Zealand. Auckland, New Zealand: Pearson.

Grint, K. (2000). The Arts of Leadership. New York: Oxford University Press.

Grint, K. (2005). Leadership: Limits and Possibilities. New York: Palgrave Macmillan.

Guba, E. G., \& Lincoln, Y. S. (2005). Paradigmatic Controversies, Contradictions, and Emerging Confluences. In N. K. Denzin \& Y. S. Lincoln (Eds.), The Sage Handbook of Qualitative Research (3 ed., pp. 191-215). Thousand Oaks, CA: Sage. 
Hansen, H., Ropo, A., \& Sauer, E. (2007). Aesthetic Leadership. The Leadership Quarterly, 18, 544-560.

Hill, S. E. K. (2004). Team Leadership. In P. G. Northouse (Ed.), Leadership Theory and Practice (3 ed., pp. 203-233). Thousand Oaks, CA: Sage.

Hofstede, G., \& Bond, M. H. (1988). The Confucius Connection: from Cultural Roots to Economic Growth. Organizational Dynamics, 16(4), 5-21.

Hosking, D. M. (1988). Organizing, Leadership and Skilful Process. Journal of Management Studies, 25(2), 147-166.

Irurita, V. F. (1996). Optimizing: A Leadership Process for Transforming Mediocrity to Excellence. A Study of Nursing Leadership. In K. W. Parry (Ed.), Leadership Research and Practice (pp. 125-138). South Melbourne, Australia: Pitman Publishing.

Kan, M. M., \& Parry, K. W. (2004). Identifying Paradox: A Grounded Theory of Leadership in Overcoming Resistance to Change. The Leadership Quarterly, 15(4), 467-491.

Kavaliku, L. (2006). Pasifika Leadership: An Issue of Quality and Relevance. Hamilton, New Zealand: Massey University.

Knights, D., \& Willmott, H. (2007). Management and Leadership. In D. Knights \& H. Willmott (Eds.), Introducing Organizational Behaviour and Management (pp. 258-307). London: Thomson.

Korac-Kakabadse, A., \& Korac-Kakabadse, N. (1998). Leadership in Government: Study of the Australian Public Service. England: Ashgate Publishing Ltd.

Loverd, R. A. (1997). Leadership for the Public Service: Power and Policy in Action. Upper Saddle River, New Jersey: Prentice Hall.

MacMillian, W. (2006). The Power of Social Capital [Electronic Version]. $\begin{array}{lllll}\text { Retrieved } & 1 & \text { May } & 2008 & \text { from }\end{array}$ http:/ / harvardbusinessonline.hbsp.harvard.edu/b01/en/common/ite m_detail.jhtml?id=U0606A.

Marshall, C., \& Rossman, G. B. (2006). Designing Qualitative Research (4 ed.). Thousand Oaks, CA: Sage Publications. 
MPIA. (1999). Pacific Directions Report - A Report to Government on a Possible Pathway for Achieving Pacific Peoples' Aspirations. Wellington, New Zealand: Ministry of Pacific Island Affairs (MPIA).

MPIA. (2005). Pathways To Leadership: Goal 2010 - A Report on Pacific Leadership in the Public Service. Wellington, New Zealand: Ministry of Pacific Island Affairs (MPIA).

MPIA. (2006). Pacific Analysis Framework. Wellington, New Zealand: Ministry of Pacific Island Affairs (MPIA).

Mumford, M. D., Marks, M. A., Connelly, M. S., Zaccaro, S. J., \& Reiter-Palmon, R. (2000). Development of Leadership Skills: Experience and Timing. The Leadership Quarterly, 11(1), 87-114.

Murrel, K. L. (1997). Emergent Theories of Leadership for the Next Century: Towards Relational Concepts. Organizational Development Journal, 15(3), $35-42$.

Northouse, P. G. (2004). Leadership Theory and Practice. Thousand Oaks, California: Sage.

NZAID. (2008). Pacific Regional Governance - New Zealand International Aid and Development Agency (NZAID). Retrieved 8 October 2008, from http://www.nzaid.govt.nz/programmes/r-pac-governance.html

O'Leary, Z. (2004). The Essential Guide To Doing Research. London: Sage

Parker, R., \& Bradley, L. (2000). Organisational Culture in the Public Sector: Evidence from Six Organisations. The International Journal of Public Sector Management, 13(2/3), 125-141.

Parry, K. W. (1998). Grounded Theory and Social Process: A New Direction for Leadership Research. Leadership Quarterly, 9(1), 85-105.

Parry, K. W. (1999). Enhancing Adaptability: Leadership Strategies to Accommodate Change in Local Government Settings. Journal of Organizational Change, 12(2), 134-156.

Parry, K. W. (2002). Four Phenomenologically Determined Social Processes of Organizational Leadership: Further Support for the Construct of Transformational Leadership. In B. J. Avolio \& F. J. Yammarino (Eds.), 
Transformational and Charismatic Leadership: The Road Ahead (pp. 339-372).

Oxford, UK: Elsevier.

Parry, K. W., \& Proctor-Thomson, S. B. (2003). Leadership, Culture and Performance: The Case of the New Zealand Public Sector. Journal of Change Management, 3(4), 376-399.

Parry, K. W., \& Proctor, S. B. (2000). The New Zealand Leadership Survey 1999 (No. 0-475-11071-4). Wellington, New Zealand: Centre for the Study of Leadership, Victoria University of Wellington.

Parry, K. W., \& Proctor, S. B. (2001). Organisational Culture, Integrity and Developing Leadership Capability in the New Zealand Public Sector. Public Sector, 24(1), 5-10.

Patton, M. Q. (2002). Qualitative Research E Evaluation Methods (3 ed.). Thousand Oaks, CA: Sage Publications.

Perakyla, A. (2005). Analyzing Talk and Text. In N. K. Denzin \& Y. S. Lincoln (Eds.), The Sage Handbook of Qualitative Research (3 ed., pp. 869-885). Thousand Oaks: Sage.

Pfeifer, D. (2006). LEADERSHIP: Maori Leadership - From Good to Great; Modesty, Patience and the Ability to "Weave People Together" are Essential Components of Strong Maori Leadership. New Zealand Management, Jul 2006, 36.

Podger, A., Simic, A., Halton, J., Shergold, P., \& Maher, T. (2004). Integrated Leadership System in the Australian Public Service. Australian Journal of Public Administration, 63(4), 108-118.

Podsakoff, P. M., MacKenzie, S. B., \& Ahearne, M. (1997). Moderating Effects of Goal Acceptance on the Relationship Between Group Cohesiveness and Productivity. Journal of Applied Psychology, 82(6), 974-983.

Rodney, F. (1988). How Organizational Love can Improve Leadership. Organizational Dynamics, 16(4), 41-51.

Rost, J. C. (1993). Leadership for the Twenty-First Century. Westport (C.T), United States: Praeger Publishers. 
Sampson, H. (2004). Navigating the Waves: The Usefulness of a Pilot in Qualitative Research. Qualitative Research, 4(3), 383-402.

Sanga, K. F., \& Walker, K. D. (2005). Apem Moa Solomon Islands Leadership. Wellington, New Zealand: Victoria University's Institute of Research and Development in Maori and Pacific Education

Snively, S., \& Roche, B. (2000). Public Sector Leadership Best Practice Survey Report: Survey of 52 Chief Executives in New Zealand Public Sector Organisations. New Zealand: Pricewaterhouse Coopers.

SSC. (1993). Pacific Islands Participation Strategies for Government Department.

Wellington, New Zealand: State Services Commission (SSC).

SSC. (2002). Career and Development Survey 2000 - Results for the New Zealand Public Service. Retrieved 5 December 2007, from http:/ / www.ssc.govt.nz. SSC. (2004). EEO Progress in the Public Service with Special Focus on Pacific Peoples (Report of Equal Employment Opportunity, EEO, in the New Zealand Public Service Employees). New Zealand: State Services Commission (SSC).

SSC. (2005). EEO Progress in the Public Service (Report of Equal Employment Opportunity, EEO, in the New Zealand Public Service Employees). New Zealand: State Services Commission (SSC).

Stake, R. E. (2005). Qualitative Case Studies. In N. K. Denzin \& Y. S. Lincoln (Eds.), The Sage Handbook of Qualitative Research (3 ed., pp. 443-466). Thousand Oaks, CA: Sage.

Statistics, N. Z. (2008a). QuickStats About New Zealand's Population and Dwellings Retrieved 8 October, 2008, from http://www.stats.govt.nz/census/2006-census-data/quickstats-aboutnzs-pop-and-dwellings/quickstats-about-nzs-pop-and-dwellings-

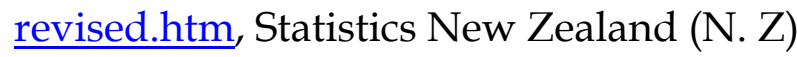

Statistics, N. Z. (2008b). QuickStats About Pacific Peoples 2006 Census Retrieved 8 October, 2008, from http://www.stats.govt.nz/census/2006census-data/quickstats-about-pacific-peoples/quickstats-about-pacificpeoples.htm, Statistics New Zealand (N. Z) 
Stogdill, R. M. (1974). Handbook of Leadership: A Survey of Theory and Research. New York: Free Press

Strauss, A., \& Corbin, J. (1990). Basics of Qualitative Research: Grounded Theory Procedures and Techniques. Newbury Park, CA: Sage

Terry, L. D. (1995). Leadership of Public Bureaucracy: The Administrator as Conservator. California, USA: Sage

Tmetuchi, R. (1998). The Bai and the Chief in Palau In D. Shuster, P. Larmour \& K. Strokirch (Eds.), Leadership in the Pacific Islands: Tradition and the Future (pp. 7-16). Canberra, Australia: National Centre for Development Studies.

Tolich, M., \& Davidson, C. (1999). Starting Fieldwork: An Introduction to Qualitative Research in New Zealand. Auckland, New Zealand: Oxford University Press.

Uhl-Bien, M. (2006). Relational Leadership Theory: Exploring the Social Processes of Leadership and Organizing. The Leadership Quarterly, 17(6), 654-676.

Van Wart, M. (2005). Dynamics of Leadership in Public Service Theory and Practice. New York: M.E. Sharpe.

Yin, R. K. (2003). Case Study Research: Design and Methods (3 ed. Vol. 5). Thousand Oaks, CA: Sage Publications.

Yukl, G. (2006). Leadership in Organizations (6 ed.). New Jersey: Pearson Prentice Hall. 


\section{APPENDIX A}

\section{Interview Schedule}

\section{Personal Details}

- Gender

- Place of birth

- Pacific Nation(s) that you self-identify with

- Years in the New Zealand Public Service

- Current position in the organisation

- Age

\section{Questions relating to leadership background}

- Can you share with me any leadership memories that you may reflect on?

- Were you taught about 'how to lead' or how to do leadership?

\section{Questions relating to participants' perceptions of leadership}

- What does leadership mean to you?

- How has this leadership idea been evidenced in your organisation?

- Who do you think should be involved in leadership?

- Why do you think leadership is important to your work and to organisational outcome?

- In your organisation what situations do you think that leadership is wanted by members but is missing? Why and how?

- If you were asked to run a leadership course in this organisation, what content would you include?

\section{Questions relating to participants' leadership actions}

- From your daily activities, which actions do you consider as leadership activities? Why?

- What guides you in doing these leadership activities?

- What do you hope to achieve through your leadership actions? 


\section{Questions relating to participants' social processes in leadership}

-What type of people do you need to work with to do your jobs better?

- How do you interact with other workers?

- How do you encourage people around you to feel motivated?

- Who has the most impact on your attitudes and motivation at work? How?

- What do you perceive as the strengths and weaknesses of dealing with people at all levels in this organisation?

Questions relating to participants' perceptions of leadership processes in organisation

- How decisions about what to do and how to do things are made here?

- What is easy and what is hard about these decision making processes?

- If your organisation is undergoing a particular change process, what people do you think might help the organisation to get through this particular change?

- How have they had these effects?

- How do these changes affect your motivation and attitude to work?

\section{Questions relating to participants' perceptions of culture}

- How does your cultural background affect your actions and participations at work?

- How do you feel about working with Palangi people in a non-Pacific environment?

- Do you feel that being of Pacific descent affects your leadership experience in any way in New Zealand?

\section{Questions relating to participants' leadership support}

- What support for leadership is available from your organisation?

- What type of support would be most helpful for Pacific public servants? 


\section{APPENDIX B}

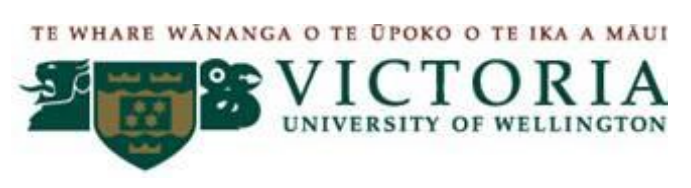

Information for participants

The leadership processes of Pacific public servants in Aotearoa, New Zealand

This research examines the leadership processes currently employed by Pacific public servants in the New Zealand Public Service. Leadership processes are dynamic actions that persuade people to move to a more desirable situation or persuade people to produce significant change resulting from social interactions between members within a context. The interview will focus on topics such as how you think about leadership, your leadership experiences at work, your perceptions on critical processes of leadership, and your perceptions on the impact of your cultural background and organisational context on leadership.

My name is Mele Katea Paea and I am the principal researcher. I am from Tonga and I am here in New Zealand undertaking a Masters degree in Management Studies at Victoria University of Wellington (VUW). This study is undertaken to fulfil the research requirement for my Masters degree.

I am inviting both male and female Pacific public servants from different positions 4 who are of Pacific descent, who have at least one year's working experience, and work permanently in the New Zealand Public Service to participate in this study.

This information sheet is provided to make sure that you, as participants of this research project, are informed as much as possible about the purpose of the research, what it means for you, and your right to confidentiality.

\footnotetext{
${ }^{4}$ Chief Executives, Directors or Managers, Chief Advisors, Accountants, senior staff, and professional staff such as policy analysts, communications advisors, finance officers, and administrators.
} 


\section{How will you be affected?}

- Your participation is completely voluntary. As a small token of my appreciation for your time and contribution I would like to offer you a coffee, lunch, or a snack at the conclusion of your interview.

- Each interview session may run for about one and a half hours.

- I will talk to you individually and the interviews will be confidential. Your name will not be shown on any reported findings and any information provided will be kept confidential to the researcher and supervisors. If you decide you do not wish to continue participating, you may withdraw from the interview before the analysis process by 30 July 2008 .

- Everyone involved in this research must sign a consent form where they can indicate how they want information received from them to be handled.

- All written data from this project will be kept in a locked file and will be restricted to the researcher and supervisors. All electronic information will be stored in a password-protected file and will be restricted to the researcher. All data from this project will be retained confidentially for three years from the completion of the Thesis (October 2008 - October 2011) for clarification purposes, and will be destroyed immediately in October 2011. A copy of this Thesis will be kept at the VUW library and the final report will be published in the form of an article. The information you provide will be used for this research project and possibly for future PHD research that may develop from the findings of this research project.

- Participants' clarification on interview notes will be sought (by emails and telephones) after the interview process to ensure that the meaning captured was, indeed, the intended thoughts conveyed by them. You will also be given a chance to request a summary of the final write up.

- Due to the small sample size and very specific characteristics of the participants, it should be noted that participants might be identifiable. However I will do everything possible to ensure confidentiality.

\begin{tabular}{|ll|l|}
\hline \multicolumn{2}{|c|}{ CONTACT INFORMATION } \\
\hline RESEACHER & PRINCIPAL SUPERVISOR \\
Name & $:$ Mele Katea Paea & Name : Dr. Deborah Jones \\
Home Phone & $:(04)$ 4766128 & Address : Victoria Management School \\
Mobile & $: 02102397182$ & Telephone : 64-4-4635731 \\
Email & $:$ paeamele@gmail.com & Email : deborah.jones@vuw.ac.nz \\
\hline
\end{tabular}




\section{APPENDIX C}

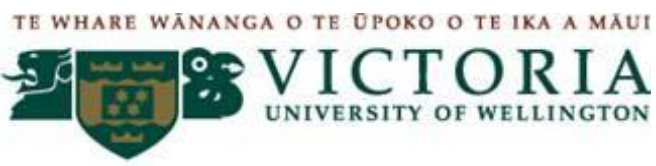

Participation Consent Form

The leadership processes of Pacific public servants in Aotearoa, New Zealand

The purpose of this agreement is to indicate your consent to participate in this research project, and your right to confidentiality.

Please confirm your consent to participate in this research by placing a tick in the relevant boxes below:

I have been given an information sheet containing the nature and objectives of this research project and I have understood that information. I have been given an opportunity to ask questions and have them answered to my satisfaction.

I am requesting a summary of the final write up.

Email or Post (Please circle and provide details in the space below)

I agree to take part in this research project.

Participant

Name:

Signature:

Date:
Date:

Researcher

Name:

Signature: 


\section{APPENDIX D}

\section{SUMMARY AND CODING SHEET}

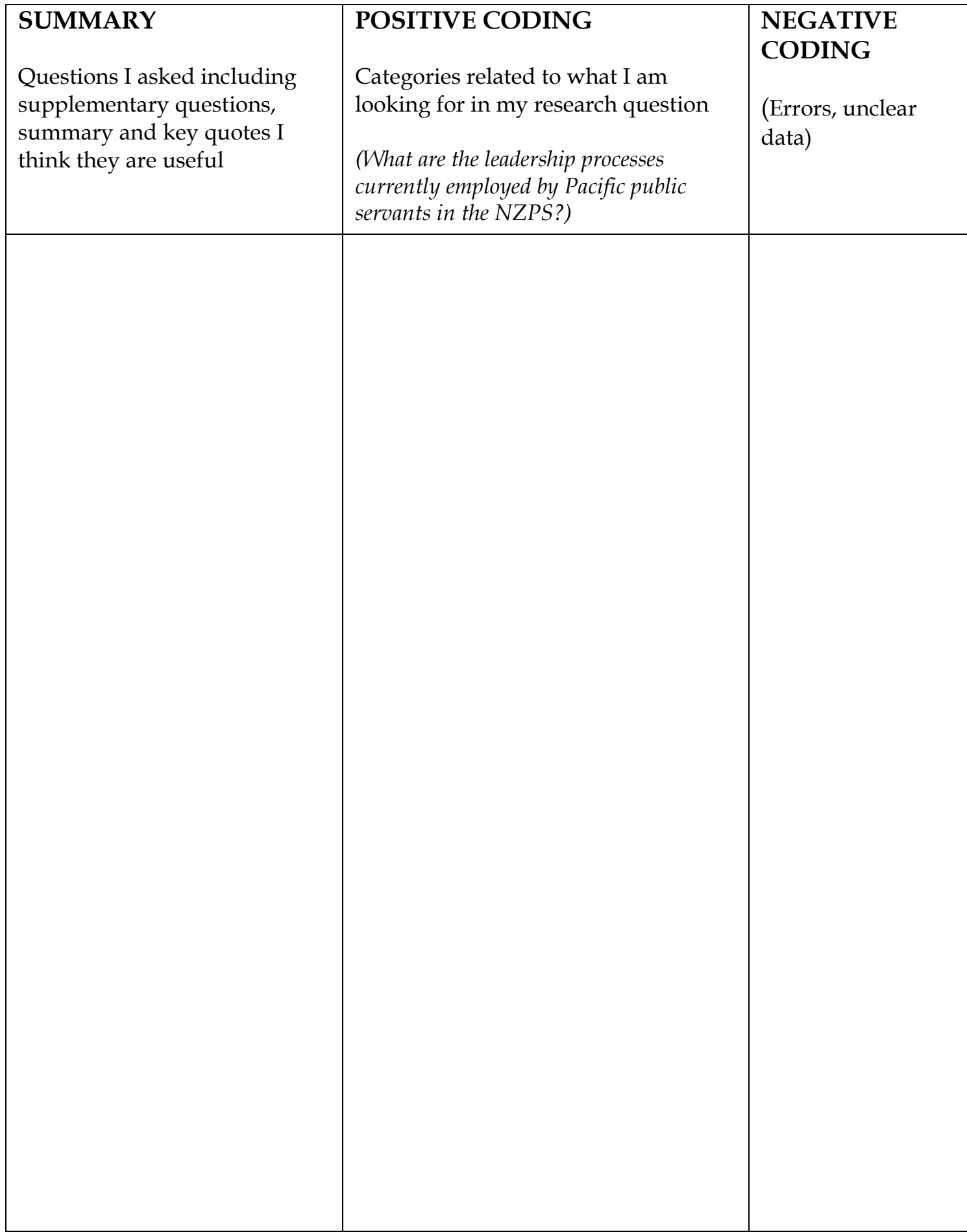

